\title{
A QUANTITATIVE VERSION OF THE ABSOLUTE SUBSPACE THEOREM
}

\author{
Jan-Hendrik Evertse (Leiden) \\ and \\ Hans Peter Schlickewei (Marburg)
}

\section{Introduction}

The celebrated Subspace Theorem of W. M. Schmidt [12] says the following:

SUBSPACE THEOREM. Let $L_{1}, \ldots, L_{n}$ be linearly independent linear forms in $n$ variables, with real or complex algebraic coefficients. Suppose $\delta>0$. Consider the inequality

$$
\left|L_{1}(\boldsymbol{x}) \ldots L_{n}(\boldsymbol{x})\right|<\|\boldsymbol{x}\|^{-\delta} \quad \text { in } \quad \boldsymbol{x} \in \mathbb{Z}^{n}
$$

where $\|\boldsymbol{x}\|=\left(x_{1}^{2}+\ldots+x_{n}^{2}\right)^{1 / 2}$. Then there are finitely many proper linear subspaces $T_{1}, \ldots, T_{A}$ of $\mathbb{Q}^{n}$ such that the set of solutions $\boldsymbol{x}$ of (1.1) is contained in

$$
T_{1} \cup \ldots \cup T_{A}
$$

Schmidt derived the Subspace Theorem as a consequence of a result on integral points in certain parallelepipeds, the so called Parametric Subspace Theorem. In fact, suppose $Q \geq 1$. Let $\boldsymbol{c}=\left(c_{1}, \ldots, c_{n}\right)$ be a tuple of real numbers with

$$
c_{1}+\ldots+c_{n}=0 \text {. }
$$

Define the set

$$
\Pi(Q, \boldsymbol{c})=\left\{\boldsymbol{x} \in \mathbb{R}^{n}|| L_{i}(\boldsymbol{x}) \mid \leq Q^{c_{i}} \quad(1 \leq i \leq n)\right\}
$$

Given $\lambda>0$, put

$$
\lambda \Pi(Q, \boldsymbol{c})=\left\{\boldsymbol{x} \in \mathbb{R}^{n}|| L_{i}(\boldsymbol{x}) \mid \leq Q^{c_{i}} \lambda \quad(1 \leq i \leq n)\right\} .
$$

Then the Parametric Subspace Theorem can be stated as follows.

PARAMETRIC SUBSPACE THEOREM. Let $\boldsymbol{c}$ be a fixed tuple satisfying (1.3). Let $\delta>0$. Then there are finitely many proper linear subspaces $T_{1}, \ldots, T_{B}$ of $\mathbb{Q}^{n}$ such that for any $Q$ which is sufficiently large there exists a subspace $T_{i} \in\left\{T_{1}, \ldots, T_{B}\right\}$ with

$$
Q^{-\delta} \Pi(Q, \boldsymbol{c}) \cap \mathbb{Z}^{n} \subset T_{i}
$$


In [14], Schmidt succeeded to give an explicit and rather uniform bound for the number $A$ of subspaces needed in (1.2) to cover the set of solutions $\boldsymbol{x}$ of (1.1). Schlickewei [9] extended this result to the case when the variables $\boldsymbol{x}$ lie in an arbitrary number field $K$ and also to the case when instead of the standard absolute value we have a finite set $S$ of absolute values of $K$. The bound obtained depends in particular upon the cardinality of the set $S$. Evertse [4] derived a much improved bound for $A$ which however still depends upon the cardinality of $S$.

It turns out that the bounds one can obtain for the number $A$ of subspaces in (1.2) in the Subspace Theorem and for the number $B$ in the Parametric Subspace Theorem differ substantially. In the number field case and for a finite set $S$ of absolute values, Schlickewei [10] obtained an explicit upper bound for the number $B$ of subspaces needed in the Parametric Subspace Theorem which does not depend upon the set $S$ at all.

To quote this result, we have to introduce some notation. Recall that the set of places of $\mathbb{Q}$ equals $\mathfrak{M}(\mathbb{Q})=\{\infty\} \cup \mathbb{P}$, where $\mathbb{P}$ is the set of prime numbers. We write $\left.\right|_{\infty}$ for the ordinary absolute value on $\mathbb{Q}$, whereas for $p \in \mathbb{P}$ we write ||$_{p}$ for the $p$-adic absolute value, normalized such that $|p|_{p}=p^{-1}$. Given a number field $K$ we write $\mathfrak{M}(K)$ for the set of its places. We denote the set of archimedean places of $K$ by $\mathfrak{M}_{\infty}(K)$ and the set of finite places of $K$ by $\mathfrak{M}_{0}(K)$. For $v \in \mathfrak{M}(K)$ we write $|\quad|_{v}$ for the absolute value having $|x|_{v}=|x|_{p}$ for $x \in \mathbb{Q}$ if $v$ lies above $p \in \mathfrak{M}(\mathbb{Q})$. We further define the normalized absolute value

$$
\|\quad\|_{v}=|\quad| \begin{aligned}
& d(v) \\
& v
\end{aligned}
$$

where

$$
d(v)=\left[K_{v}: \mathbb{Q}_{p}\right] /[K: \mathbb{Q}] .
$$

Here $\mathbb{Q}_{p}$ is the completion of $\mathbb{Q}$ at $p$, and $K_{v}$ is the completion of $K$ at $v$.

Write $d=[K: \mathbb{Q}]$. Suppose that $S$ is a finite subset of $\mathfrak{M}(K)$.

Suppose that for each $v \in \mathfrak{M}(K)$ we have a set of $n$ linear forms $\left\{L_{1}^{(v)}, \ldots, L_{n}^{(v)}\right\}$ such that

$$
\left\{L_{1}^{(v)}, \ldots, L_{n}^{(v)}\right\} \subset\left\{X_{1}, \ldots, X_{n}, X_{1}+\ldots+X_{n}\right\}
$$

and such that moreover

$$
L_{1}^{(v)}=X_{1}, \ldots, L_{n}^{(v)}=X_{n} \quad \text { for } \quad v \notin S .
$$

Further let $\boldsymbol{c}=\left\{c_{i v} \mid 1 \leq i \leq n, v \in \mathfrak{M}(K)\right\}$ be a tuple of real numbers satisfying

$$
\begin{gathered}
\sum_{v \in \mathfrak{M}(K)} \sum_{i=1}^{n} c_{i v}=0, \quad \sum_{v \in \mathfrak{M}(K)} \sum_{i=1}^{n}\left|c_{i v}\right| \leq 1 \\
c_{i v}=0 \quad(i=1, \ldots, n ; v \notin S) .
\end{gathered}
$$


Given $Q \geq 1$, define the parallelepiped $\Pi_{K}(Q, \boldsymbol{c})$ as the set of points $\boldsymbol{x} \in K^{n}$ satisfying the simultaneous inequalities

$$
\left\|L_{i}^{(v)}(\boldsymbol{x})\right\|_{v} \leq Q^{c_{i v}} \quad(1 \leq i \leq n, v \in \mathfrak{M}(K)) .
$$

For $\lambda>0$ define the dilatation of $\Pi_{K}(Q, \boldsymbol{c})$ by the factor $\lambda$ as the set of points $\boldsymbol{x} \in K^{n}$ satisfying

$$
\begin{gathered}
\left\|L_{i}^{(v)}(\boldsymbol{x})\right\|_{v} \leq Q^{c_{i v}} \lambda^{d(v)} \quad\left(1 \leq i \leq n, v \in \mathfrak{M}_{\infty}(K)\right), \\
\left\|L_{i}^{(v)}(\boldsymbol{x})\right\|_{v} \leq Q^{c_{i v}} \quad\left(1 \leq i \leq n, v \in \mathfrak{M}_{0}(K)\right),
\end{gathered}
$$

where the exponent $d(v)$ in (1.14) is as in (1.8). We write more briefly $\lambda \Pi_{K}(Q, \boldsymbol{c})$ for the set of points given by (1.14), (1.15).

Now Schlickewei's result [10] reads as follows.

Suppose $\delta>0$. For $Q \geq 1$ let $\Pi_{K}(Q, \boldsymbol{c})$ be defined as in (1.13). In particular assume that we have (1.9) - (1.12). Write $\mathfrak{D}_{K}$ for the absolute value of the discriminant of $K$. Then, there are proper linear subspaces $T_{1}, \ldots, T_{t}$ of $K^{n}$, where

$$
t \leq 2^{2^{22 n} \delta^{-2}}
$$

with the following property:

For every $Q$ satisfying

$$
Q>\max \left\{n^{2 / \delta}, \mathfrak{D}_{K}^{2 / d}\right\}
$$

(and some technical hypothesis which has no relevance in our context)

there exists a subspace $T_{i} \in\left\{T_{1}, \ldots, T_{t}\right\}$ such that

$$
Q^{-\delta} \Pi_{K}(Q, \boldsymbol{c}) \subset T_{i}
$$

In [11], Schlickewei applied this result as follows.

Let $a_{1}, \ldots, a_{n}$ be elements in $K^{*}$. Let $G$ be a multiplicative subgroup of $K^{*}$ of finite rank $r$. Consider the equation

$$
a_{1} x_{1}+\ldots+a_{n} x_{n}=1
$$

in $x_{1}, \ldots, x_{n} \in G$. Then the number of nondegenerate solutions of (1.19) (i.e., solutions such that no proper subsum on the left hand side of (1.19) vanishes) is below a bound $c(n, r, d)$, where $c(n, r, d)$ is an explicit function which depends only upon the dimension $n$, the rank $r$ of the group $G$ and the degree $d$ of $K$.

It has been known for some time, that any quantitative version of the Parametric Subspace Theorem where it is possible to avoid the term $\mathfrak{D}_{K}^{2 / d}$ in hypothesis (1.17), would imply 
an upper bound of type $c(n, r)$ for the number of solutions of (1.19), i.e., a bound which does not depend upon $d$.

It is the purpose of the present paper to prove such a version of the Subspace Theorem.

THEOREM 1.1. Let $K$ be a number field. Let $S$ be a finite subset of $\mathfrak{M}(K)$. For each $v \in \mathfrak{M}(K)$ let $\left\{L_{1}^{(v)}, \ldots, L_{n}^{(v)}\right\}$ be a subset of $\left\{X_{1}, \ldots, X_{n}, X_{1}+\ldots+X_{n}\right\}$. Assume that we have (1.10).

Suppose that

$$
0<\delta<1
$$

and let $\boldsymbol{c}=\left(c_{i v} \mid i=1, \ldots, n ; v \in \mathfrak{M}(K)\right)$ be a tuple with (1.11), (1.12). Define $\Pi_{K}(Q, \boldsymbol{c})$ as in (1.13).

Then there are proper linear subspaces $T_{1}, \ldots, T_{t}$ of $K^{n}$ where

$$
t=t(n, \delta) \leq 4^{(n+9)^{2}} \delta^{-n-4}
$$

with the following property:

For any $Q$ with

$$
Q \geq n^{2 / \delta}
$$

there exists a subspace $T_{i} \in\left\{T_{1}, \ldots, T_{t}\right\}$ with

$$
Q^{-\delta} \Pi_{K}(Q, \boldsymbol{c}) \subset T_{i}
$$

The consequences for equation (1.19) will be derived in a subsequent paper [5].

In the proof of his result (1.16) - (1.18), Schlickewei used the generalization of Minkowski's second theorem on convex bodies, as derived independently by McFeat [7] and by Bombieri and Vaaler [1]. This generalization gives an upper and a lower bound for the product of the successive minima of a convex body in $\mathbb{A}_{K}^{n}$, where $\mathbb{A}_{K}$ is the ring of adèles of $K$. The quotient of the upper bound and the lower bound is equal to $c(n) \mathfrak{D}_{K}^{n / 2 d}$ with some function $c(n)$ of $n$. It is the dependence on $\mathfrak{D}_{K}$ of this quotient that is responsible for the occurrence of the term with the discriminant in (1.17).

In the current paper we apply the recent "absolute" Minkowski theorem, versions of which were obtained independently by Roy and Thunder ([8], Theorem 6.3) and, in a more general Arakelov Theory setting, by Zhang ([15], Theorem 5.8). In our paper we have used the version of Roy and Thunder since this is better adapted to our purposes. The absolute Minkowski theorem has the advantage that it does not involve any discriminant at all. However, when applying it in our proof, we have to deal with vectors whose coordinates are algebraic, but where we cannot specify the number field in which these coordinates lie. Thus, we are forced to extend all other arguments in our proof so that we 
can handle arbitrary vectors in $\overline{\mathbb{Q}}^{n}$ instead of just vectors in $K^{n}$ for some fixed number field $K$. At the end, we arrive at a result which is much more general than the classical Subspace Theorem, in fact we obtain "absolute" generalizations of both the Parametric Subspace Theorem and the Subspace Theorem, dealing with vectors $\boldsymbol{x}$ in $\overline{\mathbb{Q}}^{n}$ rather than in $K^{n}$.

In the next two sections we will give the absolute generalisations of both the Parametric Subspace Theorem and the Subspace Theorem and not just for forms in $\left\{X_{1}, \ldots, X_{n}\right.$, $\left.X_{1}+\ldots+X_{n}\right\}$ but for arbitrary linear forms.

Another feature of Theorem 1.1 is the much better upper bound (1.21) for the number of subspaces as compared with the upper bound (1.16) in Schlickewei's result. This is due to the fact that in his proof, Schlickewei applied Roth's Lemma, whereas in our deduction of Theorem 1.1 we use the improvement of Roth's Lemma obtained by Evertse [3] by making explicit the arguments in the proof of Faltings' Product Theorem [6].

We point out however that the removal of the discriminant term from the lower bound of $Q$ (cf. (1.17), (1.22)) is due only to the use of the absolute Minkowski theorem and has nothing to do with the improvement of Roth's lemma. In fact, also with the old Roth lemma we would have obtained a result with a lower bound for $Q$ as in (1.22). But the upper bound for the number of subspaces would have become doubly exponential in $n$.

\section{The absolute Parametric Subspace Theorem}

We need some further notation. We fix an algebraic closure $\overline{\mathbb{Q}}$ of $\mathbb{Q}$. All algebraic number fields occurring in this paper will be considered to be subfields of $\overline{\mathbb{Q}}$.

As in section 1 , let $K$ be a number field and $\mathfrak{M}(K)=\mathfrak{M}_{\infty}(K) \cup \mathfrak{M}_{0}(K)$ the set of places of $K$. The absolute values \|\|$_{v}$ introduced in (1.7), (1.8) satisfy the product formula

$$
\prod_{v \in \mathfrak{M}(K)}\|x\|_{v}=1 \quad \text { for } \quad x \in K^{*}
$$

If $F$ is a finite extension of $K$ and if $w \in \mathfrak{M}(F)$ lies above $v \in \mathfrak{M}(K)$, then the normalized absolute values $\|\quad\|_{w}$ and $\|\quad\|_{v}$ are related by

$$
\|x\|_{w}=\|x\|_{v}^{d(w / v)} \quad \text { for } \quad x \in K
$$

where

$$
d(w / v)=\left[F_{w}: K_{v}\right] /[F: K] .
$$

Note that for any $v \in \mathfrak{M}(K)$

$$
\sum_{w \mid v} d(w / v)=1
$$


where the sum is over all places $w \in \mathfrak{M}(F)$ lying above $v$.

In section $1,(1.13)$, we considered parallelepipeds

$$
\Pi_{K}(Q, \boldsymbol{c})=\left\{\boldsymbol{x} \in K^{n} \mid\left\|L_{i}^{(v)}(\boldsymbol{x})\right\|_{v} \leq Q^{c_{i v}} \quad(v \in \mathfrak{M}(K), i=1, \ldots, n)\right\} .
$$

There is a height function which we call the twisted height and which is closely related to $\Pi_{K}(Q, \boldsymbol{c})$. It is defined as follows.

$$
H_{K, Q, \boldsymbol{c}}(\boldsymbol{x})=\prod_{v \in \mathfrak{M}(K)} \max _{1 \leq i \leq n} \frac{\left\|L_{i}^{(v)}(\boldsymbol{x})\right\|_{v}}{Q^{c_{i v}}} \text { for } \boldsymbol{x} \in K^{n} \backslash\{\mathbf{0}\} .
$$

It is clear that $\boldsymbol{x} \in \Pi_{K}(Q, \boldsymbol{c})$ implies $H_{K, Q, \boldsymbol{c}}(\boldsymbol{x}) \leq 1$ and more generally, in view of (1.14),

$$
\boldsymbol{x} \in \lambda \Pi_{K}(Q, \boldsymbol{c}) \text { implies } H_{K, Q, \boldsymbol{c}}(\boldsymbol{x}) \leq \lambda .
$$

The forms in $(1.9),(1.10)$ defining $\Pi_{K}(Q, \boldsymbol{c})$ in $(1.13)$ are very special. We will now study a more general setting.

Let $\left\{L_{1}, \ldots, L_{r}\right\}$ be a family ${ }^{1}$ (i.e., an unordered sequence, possibly with repetitions) of linear forms in $X_{1}, \ldots, X_{n}$ with rank $\left\{L_{1}, \ldots, L_{r}\right\}=n$ and

$$
L_{i}\left(X_{1}, \ldots, X_{n}\right) \in K\left[X_{1}, \ldots, X_{n}\right] \quad(i=1, \ldots, r) .
$$

Suppose that for each $v \in \mathfrak{M}(K)$ we have a set $\left\{L_{1}^{(v)}, \ldots, L_{n}^{(v)}\right\}$ of linear forms with

$$
\left\{L_{1}^{(v)}, \ldots, L_{n}^{(v)}\right\} \subset\left\{L_{1}, \ldots, L_{r}\right\} \text { and } \operatorname{rank}\left\{L_{1}^{(v)}, \ldots, L_{n}^{(v)}\right\}=n .
$$

For $v \in \mathfrak{M}(K)$ we put

$$
\Delta_{v}=\left\|\operatorname{det}\left(L_{1}^{(v)}, \ldots, L_{n}^{(v)}\right)\right\|_{v}
$$

Furthermore, we let $\boldsymbol{c}=\left(c_{i v}\right)(v \in \mathfrak{M}(K), i=1, \ldots, n)$ be a tuple of real numbers satisfying

$$
c_{1 v}=\ldots=c_{n v}=0 \quad \text { for all but finitely many } v \in \mathfrak{M}(K)
$$

For each finite extension $F$ of $K$ and for every place $w \in \mathfrak{M}(F)$ lying above $v \in \mathfrak{M}(K)$ we define

$$
L_{i}^{(w)}=L_{i}^{(v)}, c_{i w}=d(w / v) c_{i v}, \Delta_{w}=\Delta_{v}^{d(w / v)},
$$

\footnotetext{
${ }^{1}$ We deal with families of linear forms instead of just sets since this simplifies our arguments and since it is slightly more convenient for applications.
} 
$(\mathrm{i}=1, \ldots, \mathrm{n})$, where $d(w / v)$ is as in (2.3). By (2.2), (2.9) we have $\Delta_{w}=\left\|\operatorname{det}\left(L_{1}^{(w)}, \ldots, L_{n}^{(w)}\right)\right\|_{w}$. Let $Q \geq 1$. For $\boldsymbol{x} \in \overline{\mathbb{Q}}^{n}, \boldsymbol{x} \neq \mathbf{0}$ we define the twisted height $H_{Q, \boldsymbol{c}}(\boldsymbol{x})$ as follows: We choose a finite extension $F$ of $K$ with $\boldsymbol{x} \in F^{n}$ and we put

$$
H_{Q, \boldsymbol{c}}(\boldsymbol{x})=\prod_{w \in \mathfrak{M}(F)} \max _{1 \leq i \leq n} \frac{\left\|L_{i}^{(w)}(\boldsymbol{x})\right\|_{w}}{\Delta_{w}^{1 / n} Q^{c_{i w}}} .
$$

Notice that in view of (2.11) the right hand side of (2.12) does not depend upon the particular field $F \supset K$ with $\boldsymbol{x} \in F^{n}$. Notice moreover that by (2.8) - (2.10) for $\boldsymbol{x} \neq \mathbf{0}$ all but finitely many factors in (2.12) are equal to 1 . So $H_{Q, c}$ is a well defined function on $\overline{\mathbb{Q}}^{n}$.

We remark that for the forms considered in (1.9), (1.10), for each $v \in \mathfrak{M}(K)$ we have $\Delta_{v}=1$. So for the forms (1.9), (1.10) and for $\boldsymbol{x} \in K^{n}$ the height $H_{Q, \boldsymbol{c}}(\boldsymbol{x})$ from (2.12) coincides with the height $H_{K, Q, c}(\boldsymbol{x})$ in (2.5). Thus $H_{Q, \boldsymbol{c}}$ on the one hand generalizes the height $H_{K, Q, c}$ from (2.5) to more general linear forms and on the other hand it extends it from $K^{n}$ to $\overline{\mathbb{Q}}^{n}$.

Given our family $\left\{L_{1}, \ldots, L_{r}\right\}$ of linear forms we introduce the quantity

$$
\mathcal{H}=\mathcal{H}\left(L_{1}, \ldots, L_{r}\right)=\prod_{v \in \mathfrak{M}(K)} \max _{i_{1}, \ldots, i_{n}}\left\|\operatorname{det}\left(L_{i_{1}}, \ldots, L_{i_{n}}\right)\right\|_{v}
$$

where the maximum is taken over all subsets $\left\{i_{1}, \ldots, i_{n}\right\}$ of $\{1, \ldots, r\} . \mathcal{H}$ may be viewed as some height of $L_{1}, \ldots, L_{r}$.

Our central result is as follows.

Theorem 2.1 Let $K$ be a number field. Let $\left\{L_{1}, \ldots, L_{r}\right\}$ be a family of linear forms with (2.7). Suppose that for each $v \in \mathfrak{M}(K)$ we have forms $L_{1}^{(v)}, \ldots, L_{n}^{(v)}$ with (2.8). Let $\boldsymbol{c}=\left(c_{i v}\right)(v \in \mathfrak{M}(K), i=1, \ldots, n)$ be a tuple of real numbers with (2.10) satisfying moreover

$$
\sum_{v \in \mathfrak{M}(K)} \sum_{i=1}^{n} c_{i v}=0, \quad \sum_{v \in \mathfrak{M}(K)} \max \left\{c_{1 v}, \ldots, c_{n v}\right\} \leq 1
$$

Let

$$
0<\delta \leq 1
$$

For $\boldsymbol{x} \in \overline{\mathbb{Q}}^{n}$ define $H_{Q, c}(\boldsymbol{x})$ as in (2.12). Then there are proper linear subspaces $T_{1}, \ldots, T_{t_{1}}$ of $\overline{\mathbb{Q}}^{n}$, all defined over $K$, where

$$
t_{1}=t_{1}(n, r, \delta) \leq 4^{(n+8)^{2}} \delta^{-n-4} \log (2 r) \log \log (2 r)
$$


with the following property:

For every $Q$ with

$$
Q>\max \left\{\mathcal{H}^{1 /\left(\begin{array}{l}
r \\
n
\end{array}\right)}, n^{2 / \delta}\right\}
$$

there is a subspace $T_{i} \in\left\{T_{1}, \ldots, T_{t_{1}}\right\}$ such that

$$
\left\{\boldsymbol{x} \in \overline{\mathbb{Q}}^{n} \mid H_{Q, \boldsymbol{c}}(\boldsymbol{x}) \leq Q^{-\delta}\right\} \subset T_{i} .
$$

In applications often we have the situation that the forms $L_{i}$ have coefficients in the field $K$ but that we are interested in particular in those solutions $\boldsymbol{x}$ of $H_{Q, c}(\boldsymbol{x}) \leq Q^{-\delta}$ whose components lie in a prescribed subfield $E$ of $K$. We give a Corollary which reflects this situation.

For a number field $E$ we write $\operatorname{Gal}(\overline{\mathbb{Q}} / E)$ for the Galois group of $\overline{\mathbb{Q}}$ over $E$. Given $\boldsymbol{x}=\left(x_{1}, \ldots, x_{n}\right) \in \overline{\mathbb{Q}}^{n}$ and $\sigma \in G a l(\overline{\mathbb{Q}} / E)$ we put $\sigma(\boldsymbol{x})=\left(\sigma\left(x_{1}\right), \ldots, \sigma\left(x_{n}\right)\right)$. We prove

Corollary 2.2 Let the hypotheses be the same as in Theorem 2.1. Suppose moreover that $E$ is a subfield of $K$.

Then there are proper linear subspaces $T_{1}^{\prime}, \ldots, T_{t_{1}}^{\prime}$ of $\overline{\mathbb{Q}}^{n}$, all defined over $E$, where

$$
t_{1}=t_{1}(n, r, \delta) \leq 4^{(n+8)^{2}} \delta^{-n-4} \log (2 r) \log \log (2 r)
$$

with the following property.

For every $Q$ with

$$
Q>\max \left\{\mathcal{H}^{1 /\left(\begin{array}{l}
r \\
n
\end{array}\right)}, n^{2 / \delta}\right\}
$$

there is a subspace $T_{i}^{\prime} \in\left\{T_{1}^{\prime}, \ldots, T_{t_{1}}^{\prime}\right\}$ such that

$$
\left\{\boldsymbol{x} \in \overline{\mathbb{Q}}^{n} \mid \max _{\sigma \in \operatorname{Gal}(\overline{\mathbb{Q}} / E)} H_{Q, c}(\sigma(\boldsymbol{x})) \leq Q^{-\delta}\right\} \subset T_{i}^{\prime} .
$$

Notice that any $\boldsymbol{x} \in \overline{\mathbb{Q}}^{n}$ with $\max _{\sigma \in \operatorname{Gal}(\overline{\mathbb{Q}} / E)} H_{Q, \boldsymbol{c}}(\sigma(\boldsymbol{x})) \leq Q^{-\delta}$ a fortiori satisfies $H_{Q, \boldsymbol{c}}(\boldsymbol{x}) \leq$ $Q^{-\delta}$. Therefore the only difference between Theorem 2.1 and Corollary 2.2 lies in the fact that in (2.21) the subspaces $T_{i}^{\prime}$ are defined over the subfield $E$ of $K$ and not just over $K$ as are the subspaces $T_{i}$ in (2.18).

We finally show that Theorem 1.1 is an immediate consequence of either Theorem 2.1 or Corollary 2.2.

In Theorem 1.1 we deal with sets $Q^{-\delta} \Pi_{K}(Q, \boldsymbol{c})$. By $(2.6)$ any $\boldsymbol{x} \in Q^{-\delta} \Pi_{K}(Q, \boldsymbol{c})$ satisfies $H_{K, Q, \boldsymbol{c}}(\boldsymbol{x}) \leq Q^{-\delta}$, with $H_{K, Q, \boldsymbol{c}}$ as in (2.5). Thus in order to prove Theorem 1.1 it suffices to study the solutions of $H_{K, Q, \boldsymbol{c}}(\boldsymbol{x}) \leq Q^{-\delta}$. As observed after $(2.12), H_{K, Q, \boldsymbol{c}}(\boldsymbol{x})$ is a special instance of the twisted height $H_{Q, \boldsymbol{c}}(\boldsymbol{x})$ introduced in (2.12). 
We apply Theorem 2.1 or Corollary 2.2 with $\left\{X_{1}, \ldots, X_{n}, X_{1}+\ldots+X_{n}\right\}$ in place of $\left\{L_{1}, \ldots, L_{r}\right\}$. So the parameter $r$ becomes $n+1$. Moreover the quantities $\Delta_{v}$ from (2.9) for $\left\{X_{1}, \ldots, X_{n}, X_{1}+\ldots+X_{n}\right\}$ are all equal to 1 . Similarly, by (2.13), $\mathcal{H}\left(X_{1}, \ldots, X_{n}, X_{1}+\ldots+X_{n}\right)=1$. Thus hypothesis (2.17) reduces to

$$
Q>n^{2 / \delta}
$$

i.e., to (1.22). With Theorem 2.1 or Corollary 2.2 we therefore obtain: there are proper linear subspaces $T_{1}, \ldots, T_{t}$ of $K^{n}$ where

$$
t=t(n, \delta) \leq 4^{(n+8)^{2}} \delta^{-n-4} \log (2(n+1)) \log \log (2(n+1)) \leq 4^{(n+9)^{2}} \delta^{-n-4}
$$

with the following property:

For any $Q$ with (2.22) there exists $T_{i} \in\left\{T_{1}, \ldots, T_{t}\right\}$ such that

$$
\left\{\boldsymbol{x} \in K^{n} \mid H_{K, Q, c}(\boldsymbol{x}) \leq Q^{-\delta}\right\} \subset T_{i} .
$$

Theorem 1.1 follows.

\section{The Absolute Subspace Theorem}

We now formulate a result that is more in the spirit of (1.1).

Given $\boldsymbol{x}=\left(x_{1}, \ldots, x_{n}\right) \in K^{n}$ we introduce for $v \in \mathfrak{M}(K)$ the $v$-adic norm

$$
\|\boldsymbol{x}\|_{v}= \begin{cases}\left(\left|x_{1}\right|_{v}^{2}+\ldots+\left|x_{n}\right|_{v}^{2}\right)^{d(v) / 2} & \text { for } \quad v \in \mathfrak{M}_{\infty}(K) \\ \max \left\{\left\|x_{1}\right\|_{v}, \ldots,\left\|x_{n}\right\|_{v}\right\} & \text { for } \quad v \in \mathfrak{M}_{0}(K)\end{cases}
$$

where $d(v)$ is given by (1.8). The height of $\boldsymbol{x}$ then is defined by

$$
H(\boldsymbol{x})=\prod_{v \in \mathfrak{M}(K)}\|\boldsymbol{x}\|_{v}
$$

More generally, given $\boldsymbol{x} \in \overline{\mathbb{Q}}^{n}$ we may choose a number field $K$ such that $\boldsymbol{x} \in K^{n}$. We then define $H(\boldsymbol{x})$ again by (3.2). It is an easy consequence of (2.2) - (2.4) that our definition of $H(\boldsymbol{x})$ does not depend upon the choice of $K$. For a linear form $L(\boldsymbol{X})=\alpha_{1} X_{1}+\ldots+\alpha_{n} X_{n}$ with coefficients $\alpha_{i} \in \overline{\mathbb{Q}}$ we put $H(L)=H\left(\left(\alpha_{1}, \ldots, \alpha_{n}\right)\right)$.

We quote a version of the quantitative Subspace Theorem proved by Schmidt [14]:

Let $L_{1}, \ldots, L_{n}$ be linearly independent linear forms with coefficients in an algebraic number field $K$ of degree $d$. Consider the inequality

$$
\frac{\left|L_{1}(\boldsymbol{x})\right|}{\|\boldsymbol{x}\|} \ldots \frac{\left|L_{n}(\boldsymbol{x})\right|}{\|\boldsymbol{x}\|}<\left|\operatorname{det}\left(L_{1}, \ldots, L_{n}\right)\right| H(\boldsymbol{x})^{-n-\delta}
$$


where $0<\delta<1$.

Then there are proper linear subspaces $T_{1}, \ldots, T_{t}$ of $\mathbb{Q}^{n}$ where

$$
t \leq(2 d)^{2^{26 n} \delta^{-2}}
$$

such that the set of solutions $\boldsymbol{x} \in \mathbb{Q}^{n} \backslash\{\mathbf{0}\}$ of (3.3) with

$$
H(\boldsymbol{x})>\max \left\{H\left(L_{1}\right), \ldots, H\left(L_{n}\right),(n !)^{8 / \delta}\right\}
$$

is contained in the union

$$
T_{1} \cup \ldots \cup T_{t}
$$

Comparing Corollary 2.2 with Schmidt's result, we see that in (3.3) - (3.5) the field $\mathbb{Q}$ of rational numbers plays the rôle of the field $E$ in Corollary 2.2. However in Corollary 2.2 the absolute values under consideration are normalized absolute values on the larger field $K$, or even more generally normalized extensions thereof. In contrast with this, in (3.3) we consider the absolute value | | corresponding to the place at infinity of $\mathbb{Q}$ and we then deal with a non-normalized extension of | | onto $K$.

We proceed to give the absolute generalization of Schmidt's result.

Let $E$ be a number field. Let $S$ be a finite subset of $\mathfrak{M}(E)$ and suppose that for each $v \in S$ we have linear forms $L_{1}^{(v)}, \ldots, L_{n}^{(v)}$ with coefficients in $\overline{\mathbb{Q}}$ and with

$$
\operatorname{rank}\left\{L_{1}^{(v)}, \ldots, L_{n}^{(v)}\right\}=n .
$$

For a nonzero linear form $L=\alpha_{1} X_{1}+\ldots+\alpha_{n} X_{n}$ we define the extension $E(L)$ of $E$ by

$$
E(L)=E\left(\frac{\alpha_{1}}{\alpha_{i}}, \ldots, \frac{\alpha_{n}}{\alpha_{i}}\right)
$$

where $i$ is a subscript with $\alpha_{i} \neq 0$. We suppose that for $i=1, \ldots, n$ and for $v \in S$

$$
\left[E\left(L_{i}^{(v)}\right): E\right] \leq D
$$

and moreover that

$$
H\left(L_{i}^{(v)}\right) \leq H \quad(v \in S ; i=1, \ldots, n)
$$

For $v \in S$ write \|\|$_{v}$ for the normalized absolute value on $E$ corresponding to $v$ (cf. (1.7), (1.8)). The absolute value \|\|$_{v}$ has a unique extension \|\|$_{v}^{\prime}$, say, to $\bar{E}_{v}$, the algebraic closure of the completion $E_{v}$. Fix an embedding $\tau_{v}$ of $\overline{\mathbb{Q}}$ over $E$ into $\bar{E}_{v}$. We then extend \|\|$_{v}$ from $E$ to $\overline{\mathbb{Q}}$ by putting

$$
\|x\|_{v}=\left\|\tau_{v}(x)\right\|_{v}^{\prime} \quad \text { for } x \in \overline{\mathbb{Q}} .
$$

We obtain 
Theorem 3.1 Let $E$ be a number field and suppose $S$ is a finite subset of $\mathfrak{M}(E)$ of cardinality s. Assume that for each $v \in S$ we are given linear forms $L_{1}^{(v)}, \ldots, L_{n}^{(v)}$ in $\boldsymbol{X}=\left(X_{1}, \ldots, X_{n}\right)$ satisfying (3.6) - (3.8). Suppose moreover that for each $v \in S$ the absolute value \|\|$_{v}$ is extended to $\overline{\mathbb{Q}}$ as in $(3.9)$. Let $0<\delta<1$.

Then there exist proper linear subspaces $T_{1}, \ldots, T_{t_{2}}$ of $\overline{\mathbb{Q}}^{n}$, all defined over $E$, where

$$
t_{2}=t_{2}(n, s, D, \delta) \leq(3 n)^{2 n s} 2^{3(n+9)^{2}} \delta^{-n s-n-4} \log (4 D) \log \log (4 D)
$$

with the following property.

The set of solutions $\boldsymbol{x} \in \overline{\mathbb{Q}}^{n}$ of the inequalities

$$
\prod_{v \in S} \prod_{i=1}^{n} \max _{\sigma \in G a l(\overline{\mathbb{Q}} / E)} \frac{\left\|L_{i}^{(v)}(\sigma(\boldsymbol{x}))\right\|_{v}}{\|\sigma(\boldsymbol{x})\|_{v}} \leq \prod_{v \in S}\left\|\operatorname{det}\left(L_{1}^{(v)}, \ldots, L_{n}^{(v)}\right)\right\|_{v} H(\boldsymbol{x})^{-n-\delta}
$$

and

$$
H(\boldsymbol{x})>\max \left\{n^{4 n / \delta}, H\right\}
$$

is contained in the union

$$
T_{1} \cup \ldots \cup T_{t_{2}}
$$

Comparing Theorem 3.1 with Schmidt's result quoted above we see that the rôle of $\mathbb{Q}$ in (3.3) - (3.5) now is played by the field $E$. On the other hand the compositum $F$, say, of the fields $E\left(L_{i}^{(v)}\right)(v \in S ; i=1, \ldots, n)$ replaces the field $K$. So the analogue of the degree $d$ in Schmidt's result now is $[F: E]$. By $(3.7)$ we have $[F: E] \leq D^{n s}$.

In particular Theorem 3.1 with $E=\mathbb{Q}, S=\{\infty\}$ gives the absolute generalization of Schmidt's theorem with a bound which is much better than (3.4).

Evertse [4] has proved a result like Theorem 3.1, but with solutions $\boldsymbol{x}$ restricted to lie in $E^{n}$. He obtained the bound

$$
t_{2} \leq\left(2^{60 n^{2}} \delta^{-7 n}\right)^{s} \log (4 D) \log \log (4 D) .
$$

Clearly (3.10) again is better. However instead of (3.12) Evertse has only to assume $H(\boldsymbol{x}) \geq H$.

Our paper is organized as follows.

In section 4 we treat the rationality of the subspaces in the assertions of Theorem 2.1 and Corollary 2.2.

In view of the remark in section 2 the assertion of Corollary 2.2 then will follow once we have proved Theorem 2.1.

The proof of Theorem 2.1 in turn is given in sections 5 - 19 .

Finally in sections 20 and 21 we deduce Theorem 3.1 from Corollary 2.2. On the way of the deduction of Theorem 3.1 we give in section 20 a related result on simultaneous inequalities 
(Theorem 20.1). This intermediate result may be of some independent interest, as the bound we obtain there will be independent of $s$.

The core of the paper clearly is the proof of Theorem 2.1. We have tried to make the exposition selfcontained. To give the reader a clear picture of the essential developments in comparison with the classical Subspace Theorem we proceed as far as possible along the same lines as does Schmidt in [14] and in [13] (chapter VI).

\section{Rationality of the Subspaces}

Lemma 4.1 For $\boldsymbol{x} \in \overline{\mathbb{Q}}^{n}$ let $H_{Q, \boldsymbol{c}}(\boldsymbol{x})$ be as in (2.12). Then for any $\sigma \in \operatorname{Gal}(\overline{\mathbb{Q}} / K)$ we have

$$
H_{Q, c}(\sigma(\boldsymbol{x}))=H_{Q, c}(\boldsymbol{x})
$$

Proof. Given $\boldsymbol{x} \in \overline{\mathbb{Q}}^{n}$ we choose a finite normal extension $F$ of $K$ with $\boldsymbol{x} \in F^{n}$. For a place $w \in \mathfrak{M}(F)$ and for $\sigma \in \operatorname{Gal}(\overline{\mathbb{Q}} / K)$ we write $w_{\sigma}$ for the place in $\mathfrak{M}(F)$ such that for any $x \in F$ the non-normalized absolute values ||$_{w}$ and ||$_{w_{\sigma}}$ satisfy the relation

$$
|x|_{w_{\sigma}}=|\sigma(x)|_{w}
$$

If $w$ lies above $v \in \mathfrak{M}(K)$ then so does $w_{\sigma}$. Moreover, since the extension $F / K$ is normal, we have $\left[F_{w_{\sigma}}: K_{v}\right]=\left[F_{w}: K_{v}\right]$. Therefore with our notation (2.3) we get $d\left(w_{\sigma} / v\right)=d(w / v)$. In conjunction with (2.2) we may conclude that the normalized absolute values \|\|$_{w}$ and \|\|$_{w_{\sigma}}$ satisfy

$$
\|x\|_{w_{\sigma}}=\|\sigma(x)\|_{w} \quad \text { for each } x \in F .
$$

We now fix $v \in \mathfrak{M}(K)$ and consider $w \in \mathfrak{M}(F)$ with $w \mid v$. In view of (2.11) we have

$$
L_{i}^{\left(w_{\sigma}\right)}=L_{i}^{(w)}, c_{i, w_{\sigma}}=c_{i w}, \Delta_{w_{\sigma}}=\Delta_{w}
$$

for any $\sigma \in \operatorname{Gal}(\overline{\mathbb{Q}} / K)$. Since moreover the linear forms $L_{i}$ in $(2.7)$ have coefficients in $K$ we obtain

$$
\max _{1 \leq i \leq n} \frac{\left\|L_{i}^{(w)}(\sigma(\boldsymbol{x}))\right\|_{w}}{\Delta_{w}^{1 / n} Q^{c_{i w}}}=\max _{1 \leq i \leq n} \frac{\left\|\sigma\left(L_{i}^{(w)}(\boldsymbol{x})\right)\right\|_{w}}{\Delta_{w}^{1 / n} Q^{c_{i w}}}=\max _{1 \leq i \leq n} \frac{\left\|L_{i}^{\left(w_{\sigma}\right)}(\boldsymbol{x})\right\|_{w_{\sigma}}}{\Delta_{w_{\sigma}}^{1 / n} Q^{c_{i w_{\sigma}}}} .
$$

Furthermore, given $v \in \mathfrak{M}(K)$ and $\sigma \in G a l(\overline{\mathbb{Q}} / K)$, if $w$ runs through the places of $\mathfrak{M}(F)$ lying above $v$ then so does $w_{\sigma}$. Thus we may conclude that for any $\sigma \in \operatorname{Gal}(\overline{\mathbb{Q}} / K)$

$$
\prod_{w \mid v} \max _{1 \leq i \leq n} \frac{\left\|L_{i}^{(w)}(\sigma(\boldsymbol{x}))\right\|_{w}}{\Delta_{w}^{1 / n} Q^{c_{i w}}}=\prod_{w \mid v} \max _{1 \leq i \leq n} \frac{\left\|L_{i}^{\left(w_{\sigma}\right)}(\boldsymbol{x})\right\|_{w_{\sigma}}}{\Delta_{w_{\sigma}}^{1 / n} Q^{c_{i w_{\sigma}}}}=\prod_{w_{\sigma} \mid v} \max _{1 \leq i \leq n} \frac{\left\|L_{i}^{\left(w_{\sigma}\right)}(\boldsymbol{x})\right\|_{w_{\sigma}}}{\Delta_{w_{\sigma}}^{1 / n} Q^{c_{i w_{\sigma}}}} .
$$

Taking the product over $v \in \mathfrak{M}(K)$ we get the assertion. 
Lemma 4.2 Let $F$ be a number field. Let $M$ be a subset of $\overline{\mathbb{Q}}^{n}$ such that for any $\boldsymbol{x} \in M$ and for any $\sigma \in G a l(\overline{\mathbb{Q}} / F)$ we have $\sigma(\boldsymbol{x}) \in M$. Let $T$ be a linear subspace of $\overline{\mathbb{Q}}^{n}$ with $M \subset T$. Write $T^{\prime}$ for the subspace of $\overline{\mathbb{Q}}^{n}$ generated by $T \cap F^{n}$. Then $T^{\prime}$ is defined over $F$ and

$$
M \subset T^{\prime}
$$

Proof. Since $T^{\prime}$ has a basis in $F^{n}$ it is clearly defined over $F$.

Now suppose $\boldsymbol{x} \in M$. Pick a finite normal extension $G$ of $F$ such that $\boldsymbol{x} \in G^{n}$, and let $\left\{\sigma_{1}, \ldots, \sigma_{g}\right\}$ be the Galois group of $G$ over $F$. Choose a basis $\left\{\omega_{1}, \ldots, \omega_{g}\right\}$ of $G$ over $F$. Then $\boldsymbol{x}$ can be written as

$$
\boldsymbol{x}=\omega_{1} \boldsymbol{y}_{1}+\ldots+\omega_{g} \boldsymbol{y}_{g}
$$

with $\boldsymbol{y}_{1}, \ldots, \boldsymbol{y}_{g} \in F^{n}$. Consequently we get

$$
\sigma_{i}(\boldsymbol{x})=\sigma_{i}\left(\omega_{1}\right) \boldsymbol{y}_{1}+\ldots+\sigma_{i}\left(\omega_{g}\right) \boldsymbol{y}_{g} \quad(i=1, \ldots, g) .
$$

The matrix $\left(\sigma_{i}\left(\omega_{j}\right)\right)_{1 \leq i, j \leq g}$ is invertible. Thus, $\boldsymbol{y}_{1}, \ldots, \boldsymbol{y}_{g}$ are linear combinations of $\sigma_{1}(\boldsymbol{x}), \ldots, \sigma_{g}(\boldsymbol{x})$. By hypothesis we have $\sigma_{1}(\boldsymbol{x}), \ldots, \sigma_{g}(\boldsymbol{x}) \in M \subset T$. We may conclude that $\boldsymbol{y}_{1}, \ldots, \boldsymbol{y}_{g} \in T$ and therefore

$$
\boldsymbol{y}_{1}, \ldots, \boldsymbol{y}_{g} \in T \cap F^{n} \subset T^{\prime}
$$

In view of (4.1) we may infer that $\boldsymbol{x} \in T^{\prime}$, and the Lemma follows.

We are now in a position to prove for Theorem 2.1 and Corollary 2.2 the respective assertions on the rationality of the subspaces, assuming that all other assertions are true. In each case we apply Lemma 4.2 .

As for Theorem 2.1, $M$ is replaced by $\left\{\boldsymbol{x} \in \overline{\mathbb{Q}}^{n} \mid H_{Q, c}(\boldsymbol{x}) \leq Q^{-\delta}\right\}$ with $Q$ fixed and $F$ is replaced by $K$. By Lemma 4.1 and by (2.18) the hypotheses of Lemma 4.2 are satisfied. Thus the subspaces $T_{i}$ in Theorem 2.1 may be chosen such as to be defined over $K$.

Finally, we turn to Corollary 2.2.

The rôle of $M$ now is played by $\left\{\boldsymbol{x} \in \overline{\mathbb{Q}}^{n} \mid \max _{\sigma \in G a l(\overline{\mathbb{Q}} / E)} H_{Q, c}(\sigma(\boldsymbol{x})) \leq Q^{-\delta}\right\}$ with $Q$ fixed and the rôle of $F$ is played by the field $E$. By (2.21) the hypotheses of Lemma 4.2 again are satisfied and therefore the subspaces $T_{i}^{\prime}$ may be chosen such as to be defined over $E$.

\section{A First Reduction}

To prove Theorem 2.1, according to section 4 it suffices to show that in (2.1), (2.18) we do not need more than $t_{1}$ subspaces $T_{i}$ of $\overline{\mathbb{Q}}^{n}$, never mind whether these are defined over $K$ or not. 
In this section we want to reduce the assertion of Theorem 2.1 further.

Proposition 5.1 In order to prove Theorem 2.1 without loss of generality we are allowed to make the following additional assumptions:

(i) The family of forms $\left\{L_{1}, \ldots, L_{r}\right\}$ satisfies

$$
L_{1}=X_{1}, \ldots, L_{n}=X_{n}
$$

(ii) There exists a subset $\mathfrak{M}_{1}$ of $\mathfrak{M}_{0}(K)$ such that

$$
\mathfrak{M}_{0}(K) \backslash \mathfrak{M}_{1} \quad \text { is finite }
$$

and such that for each $v \in \mathfrak{M}_{1}$ we have

$$
L_{1}^{(v)}=X_{1}, \ldots, L_{n}^{(v)}=X_{n} ; c_{1 v}=\ldots=c_{n v}=0 .
$$

Proof. We first show that without loss of generality we may assume that there is a subset $\mathfrak{M}_{1}$ of $\mathfrak{M}_{0}(K)$ with (5.2) such that we have for each $v \in \mathfrak{M}_{1}$

$$
L_{1}^{(v)}=L_{1}, \ldots, L_{n}^{(v)}=L_{n} ; c_{1 v}=\ldots=c_{n v}=0 .
$$

Let $\mathfrak{M}_{2}$ be the subset of $\mathfrak{M}_{0}(K)$ such that for each $v \in \mathfrak{M}_{2}$

$$
c_{1 v}=\ldots=c_{n v}=0 .
$$

By $(2.10), \mathfrak{M}_{0}(K) \backslash \mathfrak{M}_{2}$ is finite.

Consider the family $\left\{L_{1}, \ldots, L_{r}\right\}$ from $(2.7)$ and write

$$
L_{i}(\boldsymbol{X})=\alpha_{i 1} X_{1}+\ldots+\alpha_{i n} X_{n} \quad(i=1, \ldots, r) .
$$

For all but finitely many $v \in \mathfrak{M}_{2}$ we have

$$
\left\|\alpha_{i j}\right\|_{v}=1
$$

for every pair $(i, j)$ with $\alpha_{i j} \neq 0$. Moreover, for all but finitely many $v \in \mathfrak{M}_{2}$ we get

$$
\left\|\operatorname{det}\left(L_{i_{1}}, \ldots, L_{i_{n}}\right)\right\|_{v}=1
$$

for any subset $\left\{i_{1}, \ldots, i_{n}\right\}$ of $\{1, \ldots, r\}$ such that $\operatorname{det}\left(L_{i_{1}}, \ldots, L_{i_{n}}\right) \neq 0$.

Let $\mathfrak{M}_{1}$ be the subset of $\mathfrak{M}_{2}$ such that for each $v \in \mathfrak{M}_{1}$ simultaneously (5.6) and (5.7) are satisfied.

Suppose $\boldsymbol{x} \in \overline{\mathbb{Q}}^{n}$ and let $F$ be a finite extension of $K$ such that $\boldsymbol{x} \in F^{n}$. We infer from (5.6) that for any $v \in \mathfrak{M}_{1}$ and for any $w \in \mathfrak{M}(F)$ with $w \mid v$ we obtain

$$
\left\|L_{i}(\boldsymbol{x})\right\|_{w} \leq \max \left\{\left\|x_{1}\right\|_{w}, \ldots,\left\|x_{n}\right\|_{w}\right\} .
$$


On the other hand applying Cramer's rule, (5.6) and (5.7), we see that for any $v \in \mathfrak{M}_{1}$, for any $w \in \mathfrak{M}(F)$ with $w \mid v$ and for any set $\left\{i_{1}, \ldots, i_{n}\right\}$ such that $\operatorname{det}\left(L_{i_{1}}, \ldots, L_{i_{n}}\right) \neq 0$

$$
\max _{1 \leq \nu \leq n}\left\|x_{\nu}\right\|_{w} \leq \max _{1 \leq \nu \leq n}\left\|L_{i_{\nu}}(\boldsymbol{x})\right\|_{w} .
$$

Combination of (5.8) and (5.9) implies that for each set $\left\{i_{1}, \ldots, i_{n}\right\}$ with (5.7), for each $v \in \mathfrak{M}_{1}$ and for each $w \in \mathfrak{M}(F)$ with $w \mid v$ we get

$$
\max _{1 \leq \nu \leq n}\left\|x_{\nu}\right\|_{w}=\max _{1 \leq \nu \leq n}\left\|L_{i_{\nu}}(\boldsymbol{x})\right\|_{w}
$$

In particular, for any pair of subsets $\left\{i_{1}, \ldots, i_{n}\right\}$ and $\left\{j_{1}, \ldots, j_{n}\right\}$ of $\{1, \ldots, r\}$ satisfying (5.7) we may infer that

$$
\max _{1 \leq \nu \leq n}\left\|L_{i_{\nu}}(\boldsymbol{x})\right\|_{w}=\max _{1 \leq \nu \leq n}\left\|L_{j_{\nu}}(\boldsymbol{x})\right\|_{w}
$$

for each $w \in \mathfrak{M}(F)$ under consideration. Our construction of $\mathfrak{M}_{1}$ is such that

$$
\mathfrak{M}_{1} \subset \mathfrak{M}_{0}(K), \mathfrak{M}_{0}(K) \backslash \mathfrak{M}_{1} \text { is finite. }
$$

Moreover for $v \in \mathfrak{M}_{1}$ we have (5.5) and (5.7). Thus, in view of (5.10) it is clear that in the definition of $H_{Q, \boldsymbol{c}}(\boldsymbol{x})$ in $(2.12)$ we may assume that for $v \in \mathfrak{M}_{1}$ the forms $\left\{L_{1}^{(v)}, \ldots, L_{n}^{(v)}\right\}$ in that ordering are always the same, $\left\{L_{1}, \ldots, L_{n}\right\}$, say. This proves our claim (5.4).

We next claim that for the family $\left\{L_{1}, \ldots, L_{r}\right\}$ we may suppose without loss of generality that

$$
L_{1}=X_{1}, \ldots, L_{n}=X_{n}
$$

To verify our claim, we show that Theorem 2.1 is invariant under linear transformations $A \in G L_{n}(K)$.

Indeed suppose $A \in G L_{n}(K)$. For a linear form $L=L(\mathbf{X})$ we define $L^{(A)}(\boldsymbol{X})=L(A \boldsymbol{X})$. The product rule for determinants and the product formula (2.1) imply that for any finite extension $F$ of $K$

$$
\prod_{w \in \mathfrak{M}(F)} \Delta_{w}^{1 / n}=\prod_{w \in \mathfrak{M}(F)}\left(\|\operatorname{det} A\|_{w} \Delta_{w}\right)^{1 / n}=\prod_{w \in \mathfrak{M}(F)}\left\|\operatorname{det}\left(\left(L_{1}^{(w)}\right)^{(A)}, \ldots,\left(L_{n}^{(w)}\right)^{(A)}\right)\right\|_{w}^{1 / n} .
$$

Moreover $\mathcal{H}$ in (2.13) remains unchanged if we replace $\left\{L_{1}, \ldots, L_{r}\right\}$ by $\left\{L_{1}^{(A)}, \ldots, L_{r}^{(A)}\right\}$. Therefore, taking in Theorem 2.1 instead of $\left\{L_{1}, \ldots, L_{r}\right\}, \boldsymbol{x}, T_{1}, \ldots, T_{t_{1}}$ respectively $\left\{L_{1}^{(A)}, \ldots, L_{r}^{(A)}\right\}, A$ we get an equivalent statement.

Our claim (5.11) now follows if for $A$ we take the inverse of the matrix $B$, where the row vectors of $B$ are the coefficient vectors of the forms $L_{1}, \ldots, L_{n}$ from (5.4). Combination of (5.4) and (5.11) finally proves Proposition 5.1. 
From now on we will always assume that (5.1) - (5.3) are satisfied.

Our next goal is to further reduce the assertion of Theorem 2.1 to a situation where in (2.9) we have

$$
\Delta_{v}=1 \quad \text { for each } v \in \mathfrak{M}(K) .
$$

In Proposition 5.2 which will be formulated below, we will make the following assumptions: We have a number field $K$ and a family $\left\{L_{1}, \ldots, L_{r}\right\}$ of linear forms in $X_{1}, \ldots, X_{n}$ of rank $n$ with

$$
L_{i}\left(X_{1}, \ldots, X_{n}\right) \in K\left[X_{1}, \ldots, X_{n}\right] \quad(i=1, \ldots, r)
$$

and

$$
L_{1}=X_{1}, \ldots, L_{n}=X_{n}
$$

For each $v \in \mathfrak{M}(K)$ we have a set $\left\{L_{1}^{(v)}, \ldots, L_{n}^{(v)}\right\}$ with

$$
\left\{L_{1}^{(v)}, \ldots, L_{n}^{(v)}\right\} \subset\left\{L_{1}, \ldots, L_{r}\right\}
$$

and

$$
\operatorname{det}\left(L_{1}^{(v)}, \ldots, L_{n}^{(v)}\right)=1
$$

Moreover we have a tuple $\boldsymbol{c}=\left(c_{i v}\right)(v \in \mathfrak{M}(K), i=1, \ldots, n)$ of real numbers with

$$
\sum_{v \in \mathfrak{M}(K)} \sum_{i=1}^{n} c_{i v}=0, \sum_{v \in \mathfrak{M}(K)} \max \left\{c_{1 v}, \ldots, c_{n v}\right\} \leq 1
$$

Finally we suppose that we have a subset $\mathfrak{M}_{1}$ of $\mathfrak{M}_{0}(K)$ such that

$$
\mathfrak{M}_{0}(K) \backslash \mathfrak{M}_{1} \text { is finite, }
$$

and such that moreover

$$
L_{1}^{(v)}=X_{1}, \ldots, L_{n}^{(v)}=X_{n} ; c_{1 v}=\ldots=c_{n v}=0 \quad \text { for } v \in \mathfrak{M}_{1}
$$

$\mathcal{H}$ and $H_{Q, \boldsymbol{c}}(\boldsymbol{x})$ are as in (2.13), (2.12) respectively.

Theorem 2.1 is a consequence of

Proposition 5.2 Suppose we have (5.12) - (5.18). Let $0<\delta<1$.

Then there are proper linear subspaces $T_{1}, \ldots, T_{t_{3}}$ of $\overline{\mathbb{Q}}^{n}$ where

$$
t_{3}=t_{3}(n, r, \delta) \leq 4^{(n+7)^{2}} \delta^{-n-4} \log (2 r) \log \log (2 r)
$$


with the following property:

For every $Q$ with

$$
Q>\max \left\{\mathcal{H}^{1 /\left(\begin{array}{l}
r \\
n
\end{array}\right)}, n^{2 / \delta}\right\}
$$

there is a subspace $T_{i} \in\left\{T_{1}, \ldots, T_{t_{3}}\right\}$ such that

$$
\left\{\boldsymbol{x} \in \overline{\mathbb{Q}}^{n} \mid H_{Q, \boldsymbol{c}}(\boldsymbol{x}) \leq Q^{-\delta}\right\} \subset T_{i} .
$$

Remark (i) The only difference between the hypotheses of Proposition 5.1 and 5.2 is (5.15).

(ii) In the deduction of Theorem 2.1 from Proposition 5.2 we will use the fact that $\left\{L_{1}, \ldots, L_{r}\right\}$ is a family and not necessarily a set. If we assume that $\left\{L_{1}, \ldots, L_{r}\right\}$ is a set, the technicalities of the deduction become more complicated.

We proceed to deduce Theorem 2.1 from Proposition 5.2.

Starting with the hypotheses we have in section 2 and assuming moreover (5.1) - (5.3) (as we may by Proposition 5.1) we will construct a finite extension $E$ of $K$ and a family of forms $\left\{M_{1}, \ldots, M_{s}\right\}$ with coefficients in $E$.

From the family $\left\{M_{1}, \ldots, M_{s}\right\}$ we will obtain for each $u \in \mathfrak{M}(E)$ sets of linear forms $\left\{M_{1}^{(u)}, \ldots, M_{n}^{(u)}\right\}$ and a tuple $\boldsymbol{e}=\left(e_{i u}\right)(u \in \mathfrak{M}(E), i=1, \ldots, n)$ such that the analogue of $(5.12)$ - (5.18) is true for $E,\left\{M_{1}, \ldots, M_{s}\right\},\left\{M_{1}^{(u)}, \ldots, M_{n}^{(u)}\right\}$, e. Denoting the corresponding twisted height by $H_{Q, e}^{\prime}(\boldsymbol{x})$, our construction will be such that

$$
H_{Q, \boldsymbol{e}}^{\prime}(\boldsymbol{x})=H_{Q, \boldsymbol{c}}(\boldsymbol{x}) \text { for all } \boldsymbol{x} \in \overline{\mathbb{Q}}^{n},
$$

where $H_{Q, c}$ is the height (2.12) we have to study in Theorem 2.1.

Combination of (5.22) and Proposition 5.2 then will imply Theorem 2.1.

To begin our construction, let $\left\{L_{1}, \ldots, L_{r}\right\}$ be the family of forms we study in Theorem 2.1. Let $\mathcal{I}$ be the collection of sets $\left\{i_{1}, \ldots, i_{n}\right\} \subset\{1, \ldots, r\}$ such that $\left\{L_{i_{1}}, \ldots, L_{i_{n}}\right\}$ is linearly independent. For $I=\left\{i_{1}, \ldots, i_{n}\right\} \in \mathcal{I}$ we put

$$
\alpha(I)=\operatorname{det}\left(L_{i_{1}}, \ldots, L_{i_{n}}\right)^{-1 / n}
$$

with a fixed choice of the $n$-th root and with $1^{1 / n}=1$. Let $E$ be the finite extension of $K$ generated over $K$ by the numbers $\alpha(I)(I \in \mathcal{I})$. Let $\left\{M_{1}, \ldots, M_{s}\right\}$ be the family of linear forms consisting of

$$
\alpha(I) L_{i} \quad(I \in \mathcal{I} ; i=1, \ldots, r) .
$$

Then we have

$$
M_{i} \in E\left[X_{1}, \ldots, X_{n}\right] \quad(i=1, \ldots, s)
$$


and

$$
s=r|\mathcal{I}| \leq r\left(\begin{array}{l}
r \\
n
\end{array}\right) \leq r^{n+1}
$$

Moreover by (5.1), $\left\{X_{1}, \ldots, X_{n}\right\} \subset\left\{M_{1}, \ldots, M_{s}\right\}$. So we may suppose that

$$
M_{1}=X_{1}, \ldots, M_{n}=X_{n} .
$$

Notice that by (2.11) we have for $u \in \mathfrak{M}(E)$ lying above $v \in \mathfrak{M}(K)$

$$
L_{i}^{(u)}=L_{i}^{(v)}, c_{i u}=d(u / v) c_{i v} \quad(i=1, \ldots, n) .
$$

We now define for $u \in \mathfrak{M}(E)$ and for $i=1, \ldots, n$

$$
M_{i}^{(u)}=\operatorname{det}\left(L_{1}^{(u)}, \ldots, L_{n}^{(u)}\right)^{-1 / n} L_{i}^{(u)} .
$$

Our definition (5.24) of $\left\{M_{1}, \ldots, M_{s}\right\}$ implies

$$
\left\{M_{1}^{(u)}, \ldots, M_{n}^{(u)}\right\} \subset\left\{M_{1}, \ldots, M_{s}\right\} \quad \text { for each } u \in \mathfrak{M}(E) .
$$

Moreover, by (5.29)

$$
\operatorname{det}\left(M_{1}^{(u)}, \ldots, M_{n}^{(u)}\right)=1 \quad \text { for each } u \in \mathfrak{M}(E) .
$$

We define the tuple $\boldsymbol{e}=\left(e_{i u}\right)(u \in \mathfrak{M}(E), i=1, \ldots, n)$

$$
e_{i u}=d(u / v) c_{i v}
$$

where $v$ is the place in $\mathfrak{M}(K)$ lying below $u$. We denote by $\mathfrak{M}_{1}(E)$ the set of places of $E$ lying above the places in $\mathfrak{M}_{1}$ (with $\mathfrak{M}_{1} \subset \mathfrak{M}_{0}(K)$ as in (5.2), (5.3)). By (5.2) we get

$$
\mathfrak{M}_{0}(E) \backslash \mathfrak{M}_{1}(E) \quad \text { is finite. }
$$

Moreover, by (5.3), (5.28), (5.29), (5.32)

$$
M_{1}^{(u)}=X_{1}, \ldots, M_{n}^{(u)}=X_{n} ; e_{1 u}=\ldots=e_{n u}=0 \quad \text { for } u \in \mathfrak{M}_{1}(E) .
$$

Finally, by (2.14) and (5.32)

$$
\sum_{u \in \mathfrak{M}(E)} \sum_{i=1}^{n} e_{i u}=0, \sum_{u \in \mathfrak{M}(E)} \max \left\{e_{1 u}, \ldots, e_{n u}\right\} \leq 1
$$

So replacing $K, \mathfrak{M}_{1},\left\{L_{1}, \ldots, L_{r}\right\},\left\{L_{1}^{(v)}, \ldots, L_{n}^{(v)}\right\}(v \in \mathfrak{M}(K)), \boldsymbol{c}=\left(c_{i v}\right)(v \in \mathfrak{M}(K)$, $i=1, \ldots, n)$ respectively by $E, \mathfrak{M}_{1}(E),\left\{M_{1}, \ldots, M_{s}\right\},\left\{M_{1}^{(u)}, \ldots, M_{n}^{(u)}\right\}(u \in \mathfrak{M}(E))$, $\boldsymbol{e}=\left(e_{i u}\right)(u \in \mathfrak{M}(E), i=1, \ldots, n)$ we see that (5.25), (5.27), (5.30), (5.31), (5.35), (5.33), (5.34) in that ordering respectively are the analogues of (5.12) - (5.18). 
We next define the height $H_{Q, \boldsymbol{e}}^{\prime}(\boldsymbol{x})$. For a finite extension $F$ of $E$ and for $w \in \mathfrak{M}(F)$ lying above $u \in \mathfrak{M}(E)$ we put, as usual,

$$
M_{i}^{(w)}=M_{i}^{(u)}, e_{i w}=d(w / u) e_{i u} \quad(i=1, \ldots, n) .
$$

Moreover, we write

$$
\Delta_{w}^{\prime}=\left\|\operatorname{det}\left(M_{1}^{(w)}, \ldots, M_{n}^{(w)}\right)\right\|_{w} \quad(w \in \mathfrak{M}(F)) .
$$

Now let $\boldsymbol{x} \in \overline{\mathbb{Q}}^{n}$ and suppose that $F$ is a finite extension of $E$ such that $\boldsymbol{x} \in F^{n}$. In complete analogy with (2.12) we define

$$
H_{Q, \boldsymbol{e}}^{\prime}(\boldsymbol{x})=\prod_{w \in \mathfrak{M}(F)} \max _{1 \leq i \leq n} \frac{\left\|M_{i}^{(w)}(\boldsymbol{x})\right\|_{w}}{\Delta_{w}^{\prime 1 / n} Q^{e_{i w}}} .
$$

Notice that by (5.31)

$$
\Delta_{w}^{\prime}=1 \quad \text { for each } w \in \mathfrak{M}(F) .
$$

Thus (5.36) becomes

$$
H_{Q, \boldsymbol{e}}^{\prime}(\boldsymbol{x})=\prod_{w \in \mathfrak{M}(F)} \max _{1 \leq i \leq n} \frac{\left\|M_{i}^{(w)}(\boldsymbol{x})\right\|_{w}}{Q^{e_{i w}}} .
$$

We may apply Proposition 5.2 to conclude that there are proper linear subspaces $T_{1}, \ldots, T_{t_{3}}$ of $\overline{\mathbb{Q}}^{n}$ where

$$
t_{3}=t_{3}(n, s, \delta) \leq 4^{(n+7)^{2}} \delta^{-n-4} \log (2 s) \log \log (2 s)
$$

with the following property:

Write $\mathcal{H}^{\prime}=\mathcal{H}\left(M_{1}, \ldots, M_{s}\right)$. Then for any $Q$ with

$$
Q>\max \left\{\mathcal{H}^{\prime 1 /\left(\begin{array}{l}
s \\
n
\end{array}\right)}, n^{2 / \delta}\right\}
$$

there is a subspace $T_{i} \in\left\{T_{1}, \ldots, T_{t_{3}}\right\}$ such that

$$
\left\{\boldsymbol{x} \in \overline{\mathbb{Q}}^{n} \mid H_{Q, e}^{\prime}(\boldsymbol{x}) \leq Q^{-\delta}\right\} \subset T_{i} .
$$

To deduce Theorem 2.1 we claim that the height $H_{Q, \boldsymbol{c}}(\boldsymbol{x})$ from (2.12) satisfies

$$
H_{Q, \boldsymbol{c}}(\boldsymbol{x})=H_{Q, e}^{\prime}(\boldsymbol{x}) \quad \text { for any } \boldsymbol{x} \in \overline{\mathbb{Q}}^{n} \text {. }
$$

We claim moreover that

$$
\mathcal{H}\left(L_{1}, \ldots, L_{r}\right)^{1 /\left(\begin{array}{l}
r \\
n
\end{array}\right)}=\mathcal{H}^{1 /\left(\begin{array}{l}
r \\
n
\end{array}\right)} \geq \mathcal{H}^{\prime 1 /\left(\begin{array}{l}
s \\
n
\end{array}\right)}=\mathcal{H}\left(M_{1}, \ldots, M_{s}\right)^{1 /\left(\begin{array}{l}
s \\
n
\end{array}\right) .}
$$

Suppose for the moment (5.41) and (5.42) to be shown. Then by (5.42), any $Q$ with (2.17) a fortiori satisfies (5.39). But then combination of (5.40) and (5.41) shows that in 
Theorem 2.1 we do not need more than $t_{3}(n, s, \delta)$ subspaces with $t_{3}$ bounded as in (5.38). All this is true under the assumption that (5.1) - (5.3) are satisfied. By Proposition 5.1 we are allowed to make this assumption. So we may conclude that in Theorem $2.1, t_{3}(n, s, \delta)$ suspaces suffice. Notice however that by (5.26) $s \leq r^{n+1}$ and so the right hand side of (5.38) is not larger than the right hand side in (2.16).

Theorem 2.1 follows.

To complete the deduction of Theorem 2.1 from Proposition 5.2, we still have to prove our claims (5.41) and (5.42).

As for (5.41), we return to (5.37). By (5.29)

$$
\left\|M_{i}^{(w)}(\boldsymbol{x})\right\|_{w}=\Delta_{w}^{-1 / n}\left\|L_{i}^{(w)}(\boldsymbol{x})\right\|_{w} \quad \text { for } \boldsymbol{x} \in F^{n} \text { and } w \in \mathfrak{M}(F) .
$$

Here $\Delta_{w}=\left\|\operatorname{det}\left(L_{1}^{(w)}, \ldots, L_{n}^{(w)}\right)\right\|_{w}$. Moreover by $(5.32)$

$$
e_{i w}=d(w / u) e_{i u}=d(w / u) d(u / v) c_{i v}=d(w / v) c_{i v}=c_{i w}
$$

for $i=1, \ldots, n$ and for $w \in \mathfrak{M}(F), w|u, u \in \mathfrak{M}(E), u| v, v \in \mathfrak{M}(K)$, i.e.,

$$
e_{i w}=c_{i w} \quad \text { for } w \in \mathfrak{M}(F) \text { and } i=1, \ldots, n \text {. }
$$

So indeed by (5.37)

$$
H_{Q, \boldsymbol{e}}^{\prime}(\boldsymbol{x})=\prod_{w \in \mathfrak{M}(F)} \max _{1 \leq i \leq n} \frac{\left\|M_{i}^{(w)}(\boldsymbol{x})\right\|_{w}}{Q^{c_{i w}}}=\prod_{w \in \mathfrak{M}(F)} \max _{1 \leq i \leq n} \frac{\left\|L_{i}^{(w)}(\boldsymbol{x})\right\|_{w}}{\Delta_{w}^{1 / n} Q^{c_{i w}}}=H_{Q, \boldsymbol{c}}(\boldsymbol{x})
$$

and assertion (5.41) is established.

As for (5.42) we prove

Lemma 5.3 Let $\left\{M_{1}, \ldots, M_{s}\right\}$ be the family of forms given by (5.24). Then

$$
\mathcal{H}\left(L_{1}, \ldots, L_{r}\right)^{1 /\left(\begin{array}{l}
r \\
n
\end{array}\right)} \geq \mathcal{H}\left(M_{1}, \ldots, M_{s}\right)^{1 /\left(\begin{array}{l}
s \\
n
\end{array}\right)} .
$$

Proof. Write $q=|\mathcal{I}|$ and let $\alpha_{1}, \ldots, \alpha_{q}$ be an enumeration of the numbers $\alpha(I)$ from (5.23) with $I \in \mathcal{I}$.

After reordering $M_{1}, \ldots, M_{s}$ we may suppose that

$$
\left\{M_{1}, \ldots, M_{s}\right\}=\left\{\alpha_{1} L_{1}, \ldots, \alpha_{q} L_{1}, \ldots, \alpha_{1} L_{r}, \ldots, \alpha_{q} L_{r}\right\}
$$

We may relabel $M_{1}, \ldots, M_{s}$ as

$$
M_{i j}=\alpha_{i} L_{j} \quad(i=1, \ldots, q ; j=1, \ldots, r) .
$$


Notice that by definition the factors $\alpha_{1}, \ldots, \alpha_{q}$ are all different from zero. Therefore we may conclude that for an $n$-tuple of pairs $\left(i_{1}, j_{1}\right), \ldots,\left(i_{n}, j_{n}\right)$

$$
\operatorname{det}\left(M_{i_{1}, j_{1}}, \ldots, M_{i_{n}, j_{n}}\right) \neq 0
$$

if and only if $\operatorname{det}\left(L_{j_{1}}, \ldots, L_{j_{n}}\right) \neq 0$, i.e., if and only if $\left\{j_{1}, \ldots, j_{n}\right\} \in \mathcal{I}$.

Now suppose $\left\{j_{1}, \ldots, j_{n}\right\} \in \mathcal{I}$. Then for $u \in \mathfrak{M}(E)$ and for any $\left\{i_{1}, \ldots, i_{n}\right\}$ with $1 \leq i_{l} \leq q(l=1, \ldots, q)$ we get

$$
\left\|\operatorname{det}\left(M_{i_{1}, j_{1}}, \ldots, M_{i_{n}, j_{n}}\right)\right\|_{u}=\left\|\alpha_{i_{1}} \ldots \alpha_{i_{n}}\right\|_{u}\left\|\operatorname{det}\left(L_{j_{1}}, \ldots, L_{j_{n}}\right)\right\|_{u} .
$$

In particular

$$
\begin{aligned}
\max _{\left(i_{1}, j_{1}\right), \ldots,\left(i_{n}, j_{n}\right)} & \left(\left\|\operatorname{det}\left(M_{i_{1}, j_{1}}, \ldots, M_{i_{n}, j_{n}}\right)\right\|_{u}\right) \leq \\
& \leq \max _{\left(i_{1}, \ldots, i_{n}\right)}\left(\left\|\alpha_{i_{1}} \ldots \alpha_{i_{n}}\right\|_{u}\right) \max _{\left(j_{1}, \ldots, j_{n}\right)}\left(\left\|\operatorname{det}\left(L_{j_{1}} \ldots L_{j_{n}}\right)\right\|_{u}\right)
\end{aligned}
$$

where the maxima are taken over $i_{l}$ and $j_{l}(l=1, \ldots, n)$ with $1 \leq i_{l} \leq q$ and $1 \leq j_{l} \leq r$. Given $l$ with $1 \leq l \leq q$, by $(5.23)$

$$
\left\|\alpha_{l}\right\|_{u} \leq \max _{\left\{j_{1}, \ldots, j_{n}\right\} \in \mathcal{I}}\left\|\operatorname{det}\left(L_{j_{1}}, \ldots, L_{j_{n}}\right)^{-1 / n}\right\|_{u} .
$$

Thus

$$
\left\|\alpha_{l}\right\|_{u} \leq\left(\min _{\left\{j_{1}, \ldots, j_{n}\right\} \in \mathcal{I}}\left\|\operatorname{det}\left(L_{j_{1}}, \ldots, L_{j_{n}}\right)\right\|_{u}\right)^{-1 / n} .
$$

We may conclude that in (5.45) the first term on the right hand side satisfies

$$
\max _{\left(i_{1}, \ldots, i_{n}\right)}\left(\left\|\alpha_{i_{1}} \ldots \alpha_{i_{n}}\right\|_{u}\right) \leq\left(\min _{\left\{j_{1}, \ldots, j_{n}\right\} \in \mathcal{I}}\left\|\operatorname{det}\left(L_{j_{1}}, \ldots, L_{j_{n}}\right)\right\|_{u}\right)^{-1}
$$

Combining (5.45) and (5.46) we may infer that

$$
\begin{aligned}
& \max _{\left.\left(i_{1}, j_{1}\right), \ldots, i_{n}, j_{n}\right)}\left(\left\|\operatorname{det}\left(M_{i_{1}, j_{1}}, \ldots, M_{i_{n}, j_{n}}\right)\right\|_{u}\right) \leq \\
& \leq \max _{\left(j_{1}, \ldots, j_{n}\right) \in \mathcal{I}}\left(\left\|\operatorname{det}\left(L_{j_{1}}, \ldots, L_{j_{n}}\right)\right\|_{u}\right) / \min _{\left(j_{1}, \ldots, j_{n}\right) \in \mathcal{I}}\left(\left\|\operatorname{det}\left(L_{j_{1}}, \ldots, L_{j_{n}}\right)\right\|_{u}\right) .
\end{aligned}
$$

Write

$$
\beta=\prod_{\left(j_{1}, \ldots, j_{n}\right) \in \mathcal{I}} \operatorname{det}\left(L_{j_{1}}, \ldots, L_{j_{n}}\right) .
$$

Then by the definition of $\mathcal{I}$ we have $\beta \neq 0$. Recall that we had $|\mathcal{I}|=q$. By (5.48) we get for any $u \in \mathfrak{M}(E)$

$$
\begin{aligned}
& \max _{\left(j_{1}, \ldots, j_{n}\right) \in \mathcal{I}}\left(\left\|\operatorname{det}\left(L_{j_{1}}, \ldots, L_{j_{n}}\right)\right\|_{u}\right) / \min _{\left(j_{1}, \ldots, j_{n}\right) \in \mathcal{I}}\left(\left\|\operatorname{det}\left(L_{j_{1}}, \ldots, L_{j_{n}}\right)\right\|_{u}\right) \leq \\
& \leq\left(\max _{\left(j_{1}, \ldots, j_{n}\right) \in \mathcal{I}}\left(\left\|\operatorname{det}\left(L_{j_{1}}, \ldots, L_{j_{n}}\right)\right\|_{u}\right)\right)^{q} /\|\beta\|_{u} .
\end{aligned}
$$


Combination of (5.47) and (5.49) yields

$$
\begin{aligned}
& \max _{\left(i_{1}, j_{1}\right), \ldots,\left(i_{n}, j_{n}\right)}\left(\left\|\operatorname{det}\left(M_{i_{1}, j_{1}}, \ldots, M_{i_{n}, j_{n}}\right)\right\|_{u}\right) \leq \\
& \leq\left(\max _{\left(j_{1}, \ldots, j_{n}\right) \in \mathcal{I}}\left(\left\|\operatorname{det}\left(L_{j_{1}}, \ldots, L_{j_{n}}\right)\right\|_{u}\right)\right)^{q} /\|\beta\|_{u} .
\end{aligned}
$$

Taking the product over $u \in \mathfrak{M}(E)$ and applying the product formula we get with (5.50)

$$
\mathcal{H}^{\prime}=\mathcal{H}\left(M_{1}, \ldots, M_{s}\right) \leq\left(\prod_{u \in \mathfrak{M}(E)}\|\beta\|_{u}^{-1}\right) \mathcal{H}\left(L_{1}, \ldots, L_{r}\right)^{q}=\mathcal{H}^{q}
$$

The assertion of Lemma 5.3 (and thus of (5.42)) is

$$
\mathcal{H}^{1 /\left(\begin{array}{l}
r \\
n
\end{array}\right)} \geq \mathcal{H}^{\prime 1 /\left(\begin{array}{l}
s \\
n
\end{array}\right)}
$$

To establish (5.52), by (5.51) it suffices to show that

$$
q\left(\begin{array}{l}
r \\
n
\end{array}\right) \leq\left(\begin{array}{l}
s \\
n
\end{array}\right)
$$

however by (5.26) and since $|\mathcal{I}|=q$ this is certainly true. The Lemma follows.

So we have reduced Theorem 2.1 to Proposition 5.2. The main part of the following sections (sections 6 - 19) deals with the proof of this Proposition.

\section{$6 \quad$ Parallelepipeds}

In this section we reduce Proposition 5.2 to an assertion about "parallelepipeds" in $\overline{\mathbb{Q}}^{n}$. Parallelepipeds already play a central rôle in Schmidt's original proof of the Subspace Theorem.

The result we are going to formulate is quite similar to Theorem 1.1.

Again we start with our number field $K$ and the family $\left\{L_{1}, \ldots, L_{r}\right\}$ of linear forms in $X_{1}, \ldots, X_{n}$ of rank $n$ with

$$
L_{i}\left(X_{1}, \ldots, X_{n}\right) \in K\left[X_{1}, \ldots, X_{n}\right] \quad(i=1, \ldots, r)
$$

satisfying

$$
L_{1}=X_{1}, \ldots, L_{n}=X_{n}
$$

For each $v \in \mathfrak{M}(K)$ we have a set $\left\{L_{1}^{(v)}, \ldots, L_{n}^{(v)}\right\}$ with

$$
\left\{L_{1}^{(v)}, \ldots, L_{n}^{(v)}\right\} \subset\left\{L_{1}, \ldots, L_{r}\right\}
$$


and

$$
\operatorname{det}\left(L_{1}^{(v)}, \ldots, L_{n}^{(v)}\right)=1
$$

We have the tuple $\boldsymbol{c}=\left(c_{i v}\right)(v \in \mathfrak{M}(K), i=1, \ldots, n)$ of real numbers with

$$
\sum_{v \in \mathfrak{M}(K)} \sum_{i=1}^{n} c_{i v}=0, \quad \sum_{v \in \mathfrak{M}(K)} \max \left\{c_{i v}, \ldots, c_{n v}\right\} \leq 1 .
$$

Finally we have a subset $\mathfrak{M}_{1}$ of $\mathfrak{M}_{0}(K)$ such that

$$
\mathfrak{M}_{0}(K) \backslash \mathfrak{M}_{1} \text { is finite }
$$

and such that moreover for each $v \in \mathfrak{M}_{1}$

$$
L_{1}^{(v)}=X_{1}, \ldots, L_{n}^{(v)}=X_{n} ; c_{1 v}=\ldots=c_{n v}=0
$$

If $F$ is a finite extension of $K$ we have put for any $w \in \mathfrak{M}(F)$ lying above $v \in \mathfrak{M}(K)$

$$
L_{i}^{(w)}=L_{i}^{(v)}, c_{i w}=d(w / v) c_{i v} \quad(i=1, \ldots, n)
$$

with $d(w / v)$ as in $(2.3)$.

Let $\boldsymbol{x} \in \overline{\mathbb{Q}}^{n}$ and suppose that indeed $\boldsymbol{x} \in F^{n}$. Then in our current situation the height $H_{Q, c}(\boldsymbol{x})$ becomes

$$
H_{Q, \boldsymbol{c}}(\boldsymbol{x})=\prod_{w \in \mathfrak{M}(F)} \max _{1 \leq i \leq n} \frac{\left\|L_{i}^{(w)}(\boldsymbol{x})\right\|_{w}}{Q^{c_{i w}}} .
$$

We define the parallelepiped $\Pi_{F}(Q, \boldsymbol{c})$ to be the set of points $\boldsymbol{x} \in F^{n}$ satisfying

$$
\left\|L_{i}^{(w)}(\boldsymbol{x})\right\|_{w} \leq Q^{c_{i w}} \quad(w \in \mathfrak{M}(F), i=1, \ldots, n) .
$$

We define the algebraic closure $\Pi(Q, \boldsymbol{c})$ of $\Pi_{K}(Q, \boldsymbol{c})$ by

$$
\Pi(Q, \boldsymbol{c})=\bigcup_{F \supset K} \Pi_{F}(Q, \boldsymbol{c})
$$

where the union is over all finite extensions $F$ of $K$.

We now fix a place

$$
v_{0} \in \mathfrak{M}_{1}
$$

For $\lambda>0$ and a finite extension $F$ of $K$ we define the dilatation of $\Pi_{F}(Q, \boldsymbol{c})$ by the factor $\lambda$ to be the set of points $x \in F^{n}$ satisfying the simultaneous inequalities

$$
\begin{aligned}
\left\|L_{i}^{(w)}(\boldsymbol{x})\right\|_{w} & \leq Q^{c_{i w}} \quad\left(w \in \mathfrak{M}(F), w \nmid v_{0}, i=1, \ldots, n\right) \\
\left\|L_{i}^{(w)}(\boldsymbol{x})\right\|_{w} & \leq Q^{c_{i w}} \lambda^{d\left(w / v_{0}\right)} \quad\left(w \in \mathfrak{M}(F), w \mid v_{0}, i=1, \ldots, n\right) .
\end{aligned}
$$


In view of (6.12) and (6.7) these become

$$
\begin{aligned}
\left\|L_{i}^{(w)}(\boldsymbol{x})\right\|_{w} \leq Q^{c_{i w}} & \left(w \in \mathfrak{M}(F), w \nmid v_{0}, i=1, \ldots, n\right) \\
\left\|x_{i}\right\|_{w} \leq \lambda^{d\left(w / v_{0}\right)} & \left(w \in \mathfrak{M}(F), w \mid v_{0}, i=1, \ldots, n\right) .
\end{aligned}
$$

We write briefly $\lambda * \Pi_{F}(Q, \boldsymbol{c})$ for the subset of points $\boldsymbol{x} \in F^{n}$ given by (6.13), (6.14). The algebraic closure of $\lambda \Pi_{k}(Q, \boldsymbol{c})$ then is defined by

$$
\lambda * \Pi(Q, \boldsymbol{c})=\bigcup_{F \supset K} \lambda * \Pi_{F}(Q, \boldsymbol{c}),
$$

where again the union is over all finite extensions $F$ of $K$. We call $\Pi(Q, \boldsymbol{c})$ the parallelepiped associated with the height $H_{Q, \boldsymbol{c}}(\boldsymbol{x})$.

Remark. Our definition of $\lambda * \Pi(Q, \boldsymbol{c})$ might seem somewhat artificial. We have "concentrated" the factor of dilatation to the places lying above $v_{0}$ with $v_{0} \in \mathfrak{M}_{1}$ simply for technical reasons. In view of Lemma 6.3 below it will become clear that the assertion we will enunciate in the following Proposition 6.1 would be true just the same if in the definition of $\lambda * \Pi(Q, \boldsymbol{c})$ instead of (6.13), (6.14) we would use the inequalities

$$
\begin{aligned}
\left\|L_{i}^{(w)}(\boldsymbol{x})\right\|_{w} & \leq Q^{c_{i w}} \lambda^{d(w)} & & \left(w \in \mathfrak{M}_{\infty}(F), i=1, \ldots, n\right) \\
\left\|L_{i}^{(w)}(\boldsymbol{x})\right\|_{w} & \leq Q^{c_{i w}} & & \left(w \in \mathfrak{M}_{0}(F), i=1, \ldots, n\right) .
\end{aligned}
$$

This definition would be the natural extension of (1.14), (1.15). We will not discuss this further. At any rate (6.13), (6.14) will smooth out certain details in our proof later on.

Proposition 6.1 Let $K$ be a number field. Suppose we have a family $\left\{L_{1}, \ldots, L_{r}\right\}$ of linear forms, for each $v \in \mathfrak{M}(K)$ a set of forms $\left\{L_{1}^{(v)}, \ldots, L_{n}^{(v)}\right\}$ and moreover a tuple $\boldsymbol{c}=\left(c_{i v}\right)(v \in \mathfrak{M}(K), i=1, \ldots, n)$ such that $(6.1)$ - (6.7) are satisfied.

For $Q \geq 1$ define the parallelepiped $\Pi(Q, \boldsymbol{c})$ with (6.8), (6.10), (6.11) and for $\lambda>0$ let $\lambda * \Pi(Q, \boldsymbol{c})$ be defined by $(6.12)-(6.14)$.

Suppose $0<\delta<1$. Then there exist proper linear subspaces $T_{1}, \ldots, T_{t_{4}}$ of $\overline{\mathbb{Q}}^{n}$ where

$$
t_{4}=t_{4}(n, r, \delta) \leq 4^{(n+6)^{2}} \delta^{-n-4} \log (2 r) \log \log (2 r)
$$

with the following property:

For each $Q$ with

$$
Q>\max \left\{\mathcal{H}^{1 /\left(\begin{array}{l}
r \\
n
\end{array}\right)}, n^{1 / \delta}\right\}
$$

there exists $i$ with $1 \leq i \leq t_{4}$ such that

$$
Q^{-\delta} * \Pi(Q, \boldsymbol{c}) \subset T_{i}
$$


It turns out that Proposition 6.1 essentially is equivalent to Proposition 5.2. Given $Q$ with (5.20) we write

$$
M(Q)=\left\{\boldsymbol{x} \in \overline{\mathbb{Q}}^{n} \mid H_{Q, c}(\boldsymbol{x}) \leq Q^{-\delta}\right\}
$$

for the set considered in (5.21). Obviously $Q^{-\delta} * \Pi(Q, \boldsymbol{c}) \subset M(Q)$. Therefore Proposition 5.2 implies a result of the type stated in Proposition 6.1 (with slightly different numerical constants in (6.16), (6.17)).

Since we want to reduce Proposition 5.2 to Proposition 6.1 we need the opposite implication.

We will prove:

Lemma 6.2 Let $\vartheta>0$ and $\lambda>0$. Then for any $\boldsymbol{x} \in \overline{\mathbb{Q}}^{n}$ with

$$
H_{Q, c}(\boldsymbol{x}) \leq \lambda
$$

there exists $\beta \in \overline{\mathbb{Q}}^{*}$ such that

$$
\beta \boldsymbol{x} \in(1+\vartheta) \lambda * \Pi(Q, \boldsymbol{c}) .
$$

Suppose for the moment Lemma 6.2 to be shown.

We proceed to deduce Proposition 5.2 from Proposition 6.1.

Suppose $0<\delta<1$. Put $\delta^{\prime}=\delta / 2$. Pick $\vartheta>0$ such that

$$
1+\vartheta \leq n
$$

Now by (5.20) we have $Q^{\delta / 2}>n$. So with $\vartheta$ from (6.22) we get for any $Q$ with (5.20)

$$
(1+\vartheta) Q^{-\delta} \leq Q^{-\delta^{\prime}}
$$

On the other hand, any $Q$ with (5.20) satisfies (6.17) with $\delta$ replaced by $\delta^{\prime}$. Let $T_{1}, \ldots, T_{t_{4}}$ be the subspaces we get in Proposition 6.1 with $\delta$ replaced by $\delta^{\prime}$. Then for any $Q$ having (5.20) we get by Proposition 6.1

$$
Q^{-\delta^{\prime}} * \Pi(Q, \boldsymbol{c}) \subset T_{i}
$$

for some $i$ with $1 \leq i \leq t_{4}\left(n, r, \delta^{\prime}\right)$.

Now consider for $Q$ with (5.20) the set $M(Q)$ introduced in (6.19). Suppose $\boldsymbol{x} \in M(Q)$. We apply Lemma 6.2 with $\lambda=Q^{-\delta}$ and with $\vartheta$ as in (6.22). Accordingly there exists $\beta \in \overline{\mathbb{Q}}^{*}$ such that

$$
\beta \boldsymbol{x} \in(1+\vartheta) Q^{-\delta} * \Pi(Q, \boldsymbol{c}) .
$$


Combination of (6.23) and (6.24) implies $\beta \boldsymbol{x} \in T_{i}$, hence $\boldsymbol{x} \in T_{i}$. We may conclude that

$$
M(Q) \subset T_{i}
$$

for some $i$ with $1 \leq i \leq t_{4}(n, r, \delta / 2)$.

However, it is easily seen that the upper bound given in (6.16) for $t_{4}(n, r, \delta / 2)$ does not exceed the upper bound we claim for $t_{3}(n, r, \delta)$ in (5.19). Proposition 5.2 follows.

We still have to prove Lemma 6.2. We use

Lemma 6.3 Let $F$ be a number field. Suppose that for $w \in \mathfrak{M}(F)$ we are given positive real numbers $A_{w}$ satisfying

$$
A_{w}=1 \quad \text { for all but finitely many } w \in \mathfrak{M}(F) \text {, }
$$

$$
\prod_{w \in \mathfrak{M}(F)} A_{w}>1
$$

Then there exist a finite extension $E$ of $F$ and an element $\beta \in E^{*}$ with the following properties:

$$
\begin{gathered}
\|\beta\|_{u} \leq A_{w}^{d(u / w)} \quad \text { for all } u \in \mathfrak{M}(E) \text { lying above } \\
w \in \mathfrak{M}(F) \text { and for all } w \in \mathfrak{M}(F), \\
\beta^{k} \in F \quad \text { for some } k \in \mathbb{N} .
\end{gathered}
$$

Proof. Let $T$ be the finite subset of $\mathfrak{M}(F)$ consisting of all archimedean places and of all places $w \in \mathfrak{M}(F)$ with $A_{w} \neq 1$. Write $G_{T}$ for the group of $T$-units in $F$. So $G_{T}=\left\{g \in F^{*} \mid\|g\|_{w}=1\right.$ for $\left.w \in \mathfrak{M}(F) \backslash T\right\}$. Suppose $T$ has cardinality $t$. Then by Dirichlet's unit theorem, $G_{T}$ is a finitely generated multiplicative group of rank $t-1$. In fact it is well known that the set of vectors $\left(\log \|g\|_{w}\right)_{w \in T}$ with $g \in G_{T}$ forms a lattice of rank $t-1$ in the subspace of $\mathbb{R}^{t}=\left\{\left(x_{w}\right)_{w \in T} \mid x_{w} \in \mathbb{R}\right\}$ defined by the equation

$$
\sum_{w \in T} x_{w}=0
$$

In particular this implies that there exists a positive constant $c$ such that for any tuple $\left(x_{w}\right)_{w \in T}$ of real numbers with (6.29) we can find an element $g \in G_{T}$ satisfying

$$
\left|\log \|g\|_{w}-x_{w}\right| \leq c \quad(w \in T) .
$$

Write $A=\prod_{w \in \mathfrak{M}(F)} A_{w}$. By (6.26) we can find a positive integer $k$ such that

$$
A^{k} \geq e^{c t}
$$


We consider the vector $\left(x_{w}\right)_{w \in T}$ with components $x_{w}=k \log A_{w}-\frac{k}{t} \log A$. We infer from the definition of $A$ and $T$ that $\left(x_{w}\right)$ satisfies (6.29). For this vector we choose $g \in G_{T}$ according to (6.30). Let $\beta$ be a $k$-th root of $g$. With this choice of $\beta,(6.28)$ is true. Write $E=F(\beta)$ and let $R$ be the set of places of $E$ lying above the places in $T$. Let $u \in R$ and suppose $w \in T$ is such that $u \mid w$. We then get using (6.3) and (6.31)

$$
\begin{aligned}
& \left|\log \|\beta\|_{u}-d(u / w)\left\{\log A_{w}-\frac{1}{t} \log A\right\}\right| \\
& \quad=\frac{1}{k}\left|\log \|g\|_{u}-d(u / w)\left\{k \log A_{w}-\frac{k}{t} \log A\right\}\right| \\
& \quad=\frac{d(u / w)}{k}\left|\log \|g\|_{w}-k \log A_{w}+\frac{k}{t} \log A\right| \\
& \quad \leq \frac{d(u / w)}{k} c \leq d(u / w) \frac{1}{t} \log A .
\end{aligned}
$$

This implies (6.27) for $u \in \mathfrak{M}(E)$ lying above some $w \in T$, i.e., for $u \in R$.

For $u \notin R$ we obtain

$$
\|\beta\|_{u}=\|g\|_{u}^{1 / k}=\|g\|_{w}^{d(u / w) / k}=1=A_{w}^{d(u / w)}
$$

(here $w$ is the place in $\mathfrak{M}(F) \backslash T$ lying below $u$ ). So (6.27) is true for $u \in \mathfrak{M}(E) \backslash R$ as well. Lemma 6.3 follows.

We now derive Lemma 6.2 from Lemma 6.3.

Let $\boldsymbol{x} \in \overline{\mathbb{Q}}^{n}$ with $H_{Q, \boldsymbol{c}}(\boldsymbol{x}) \leq \lambda$ be given. We may assume $\boldsymbol{x} \neq \mathbf{0}$. Suppose $F$ is a finite extension of $K$ such that $\boldsymbol{x} \in F^{n}$. Then by (6.9) we have

$$
\prod_{w \in \mathfrak{M}(F)} \max _{1 \leq i \leq n} \frac{\left\|L_{i}^{(w)}(\boldsymbol{x})\right\|_{w}}{Q^{c_{i w}}} \leq \lambda
$$

Put

$$
B_{w}=\max _{1 \leq i \leq n} \frac{\left\|L_{i}^{(w)}(\boldsymbol{x})\right\|_{w}}{Q^{c_{i w}}} \quad(w \in \mathfrak{M}(F)) .
$$

By (6.4), $B_{w}>0$ for each $w \in \mathfrak{M}(F)$.

We want to apply Lemma 6.2 with

$$
\begin{cases}A_{w}=B_{w}^{-1} & \text { for } w \in \mathfrak{M}(F), w \nmid v_{0} \\ A_{w}=B_{w}^{-1}\{(1+\vartheta) \lambda\}^{d\left(w / v_{0}\right)} & \text { for } w \in \mathfrak{M}(F) \text { with } w \mid v_{0}\end{cases}
$$

Notice that in view of $(6.32),(6.33)$

$$
\prod_{w \in \mathfrak{M}(F)} B_{w} \leq \lambda .
$$


Hence

$$
\prod_{w \in \mathfrak{M}(F)} A_{w}=\left(\prod_{w \in \mathfrak{M}(F)} B_{w}^{-1}\right)(1+\vartheta) \lambda>1
$$

So (6.26) is satisfied.

Moreover, by (6.6) - (6.8) for all but finitely many $w \in \mathfrak{M}(F), c_{1 w}=\ldots=c_{n w}=0$. Also for all but finitely many $w \in \mathfrak{M}(F), \max _{1 \leq i \leq n}\left\|L_{i}^{(w)}(\boldsymbol{x})\right\|_{w}=1$. Thus we may conclude that for all but finitely many $w \in \mathfrak{M}(F), \quad B_{w}=1$ and hence $A_{w}=1$. So (6.25) is satisfied as well.

By Lemma 6.3 we can find a finite extension $E$ of $F$ and an element $\beta \in E^{*}$ with

$$
\|\beta\|_{u} \leq A_{w}^{d(u / w)} \text { for } u \in \mathfrak{M}(E) \text { lying above } w \in \mathfrak{M}(F) \text { with } w \nmid v_{0} .
$$

Combination of (6.33) - (6.35) yields

$$
\max _{1 \leq i \leq n} \frac{\left\|L_{i}^{(u)}(\beta \boldsymbol{x})\right\|_{u}}{Q^{c_{i n}}}=B_{w}^{d(u / w)}\|\beta\|_{u} \leq 1
$$

for $u \in \mathfrak{M}(E)$ lying above $w \in \mathfrak{M}(F)$ with $w \nmid v_{0}$, whereas for $u \in \mathfrak{M}(E)$ lying above $w \in \mathfrak{M}(F)$ with $w \mid v_{0}$ we may infer, again with (6.33) - (6.35), that

$$
\max _{1 \leq i \leq n}\left\|\beta x_{i}\right\|_{u}=B_{w}^{d(u / w)}\|\beta\|_{u} \leq((1+\vartheta) \lambda)^{d\left(u / v_{0}\right)} .
$$

But this means $\beta \boldsymbol{x} \in(1+\vartheta) \lambda * \Pi(Q, \boldsymbol{c})$ and Lemma 6.2 follows.

To summarize, so far we have reduced the assertion of Theorem 2.1 to Proposition 6.1. Proposition 6.1 will be proved in sections 7 - 19 .

\section{Absolute Geometry of Numbers}

Let

$$
\boldsymbol{A}=\left(A_{i v} \mid v \in \mathfrak{M}(K), i=1, \ldots, n\right)
$$

be a tuple of positive real numbers with

$$
A_{1 v}=\ldots A_{n v}=1 \text { for all but finitely many } v \in \mathfrak{M}(K) .
$$

For a finite extension $F$ of $K$ and for $w \in \mathfrak{M}(F)$ lying above $v \in \mathfrak{M}(K)$ we put

$$
A_{i w}=A_{i v}^{d(w / v)}
$$

with $d(w / v)$ as in (2.3). For $v \in \mathfrak{M}(K)$ let $L_{1}^{(v)}, \ldots, L_{n}^{(v)}$ be the linear forms from (6.3), (6.4), (6.7). For $\boldsymbol{x} \in \overline{\mathbb{Q}}^{n}$ we define the twisted height $H_{\boldsymbol{A}}(\boldsymbol{x})$ as follows. If $F$ is a finite extension of $K$ such that $\boldsymbol{x} \in F^{n}$ we put

$$
H_{\boldsymbol{A}}(\boldsymbol{x})=\prod_{w \in \mathfrak{M}(F)} \max _{1 \leq i \leq n} \frac{\left\|L_{i}^{(w)}(\boldsymbol{x})\right\|_{w}}{A_{i w}} .
$$


Following Roy and Thunder [8] we introduce for the height $H_{\boldsymbol{A}}(\boldsymbol{x})$ successive minima $\lambda_{1}, \ldots, \lambda_{n}$.

For $\lambda>0$ we let $V_{\boldsymbol{A}}(\lambda)$ be the $\overline{\mathbb{Q}}$-vector space generated by the elements $\boldsymbol{x} \in \overline{\mathbb{Q}}^{n}$ with $H_{\boldsymbol{A}}(\boldsymbol{x}) \leq \lambda$. For $i=1, \ldots, n$ we define

$$
\lambda_{i}=\inf \left\{\lambda \mid \operatorname{dim} V_{\boldsymbol{A}}(\lambda) \geq i\right\} .
$$

We quote Theorem 6.3 of Roy and Thunder [8].

Proposition 7.1 Suppose $\boldsymbol{A}=\left(A_{i v} \mid v \in \mathfrak{M}(K), i=1, \ldots, n\right)$ satisfies (7.1). Suppose moreover that for each pair $(i, v) \quad(1 \leq i \leq n, v \in \mathfrak{M}(K))$ there exists $\alpha_{i v} \in K$ with

$$
A_{i v}=\left\|\alpha_{i v}\right\|_{v}
$$

For $v \in \mathfrak{M}(K)$ put

$$
A_{v}=\prod_{i=1}^{n} A_{i v}
$$

Then the successive minima $\lambda_{1}, \ldots, \lambda_{n}$ of $H_{\boldsymbol{A}}(\boldsymbol{x})$ satisfy the inequality

$$
n^{-\frac{n}{2}} \prod_{v \in \mathfrak{M}(K)} \frac{\Delta_{v}}{A_{v}} \leq \lambda_{1} \ldots \lambda_{n} \leq 2^{\frac{n(n-1)}{2}} \prod_{v \in \mathfrak{M}(K)} \frac{\Delta_{v}}{A_{v}}
$$

with $\Delta_{v}$ as in $(2.9)$.

Actually, Roy and Thunder consider a slightly different twisted height, with the Euclidean norm at the infinite places in (7.3) instead of the maximum norm, i.e., they consider

$$
\widetilde{H}_{\boldsymbol{A}}(\boldsymbol{x})=\prod_{w \in \mathfrak{M}_{\infty}(F)}\left(\sum_{i=1}^{n} \frac{\left|L_{i}^{(w)}(\boldsymbol{x})\right|_{w}^{2}}{A_{i w}^{2 / d(w)}}\right)^{\frac{d(w)}{2}} \prod_{w \in \mathfrak{M}_{0}(F)} \max _{1 \leq i \leq n} \frac{\left\|L_{i}^{(w)}(\boldsymbol{x})\right\|_{w}}{A_{i w}} .
$$

They define minima $\mu_{1}, \ldots, \mu_{n}$ with respect to $\widetilde{H}_{\boldsymbol{A}}(\boldsymbol{x})$ and prove

$$
\prod_{v \in \mathfrak{M}(K)} \frac{\Delta_{v}}{A_{v}} \leq \mu_{1} \ldots \mu_{n} \leq 2^{\frac{n(n-1)}{2}} \prod_{v \in \mathfrak{M}(K)} \frac{\Delta_{v}}{A_{v}} .
$$

Now, it follows easily from $\sum_{w \mid \infty} d(w)=1$ that

$$
H_{\boldsymbol{A}}(\boldsymbol{x}) \leq \widetilde{H}_{\boldsymbol{A}}(\boldsymbol{x}) \leq n^{1 / 2} H_{\boldsymbol{A}}(\boldsymbol{x}) .
$$

(7.6) is a consequence of (7.7) and (7.8).

For our application it will be convenient to prove that in Proposition 7.1 we may drop hypothesis (7.4). Indeed we have 
Corollary 7.2 Proposition 7.1 remains true without hypothesis (7.4).

Proof. Let $S$ be the set of places in $\mathfrak{M}(K)$ such that

$$
L_{i}^{(v)}=X_{i}, A_{i v}=1 \text { for } i=1, \ldots, n \text { and for each } v \in \mathfrak{M}(K) \backslash S .
$$

By (7.1) and (6.6), (6.7), $S$ is finite.

For each $v \in S$ we pick an element $\beta_{v} \in K$ with

$$
\left\|\beta_{v}\right\|_{v} \neq 1
$$

Suppose $\varepsilon>0$. Then we can find numbers $n_{i v} \in \mathbb{Z}, m_{i v} \in \mathbb{N}(v \in S, i=1, \ldots, n)$ such that

$$
(1+\varepsilon)^{-1} A_{i v} \leq\left\|\beta_{v}\right\|_{v}^{n_{i v} / m_{i v}} \leq(1+\varepsilon) A_{i v}
$$

Let $E$ be a finite extension of $K$ containing the numbers $\beta_{v}^{1 / m_{i v}}(v \in S, i=1, \ldots, n)$. For $q \in \mathfrak{M}(E)$ we define the linear forms $L_{i}^{(q)}$ in analogy with (6.8). Moreover we define the tuple $\boldsymbol{B}=\left(B_{i q} \mid q \in \mathfrak{M}(E), i=1, \ldots, n\right)$ by

$$
B_{i q}=\left\{\begin{array}{cl}
\left\|\beta_{v}\right\|^{\left(n_{i v} / m_{i v}\right) d(q / v)} & \text { for } q \in \mathfrak{M}(E) \text { lying above } v \in S, i=1, \ldots, n \\
1 & \text { for } q \in \mathfrak{M}(E) \text { not lying above some } \\
& v \in S, i=1, \ldots, n
\end{array}\right.
$$

Given a finite extension $F$ of $E$, for $w \in \mathfrak{M}(F)$ lying above $q \in \mathfrak{M}(E)$ we write $B_{i w}=B_{i q}^{d(w / q)} \quad(i=1, \ldots, n)$.

We define the twisted height $H_{\boldsymbol{B}}(\boldsymbol{x})$ as follows. For $\boldsymbol{x} \in \overline{\mathbb{Q}}^{n}$ let $F$ be a finite extension of $E$ such that $\boldsymbol{x} \in F^{n}$. We then put

$$
H_{\boldsymbol{B}}(\boldsymbol{x})=\prod_{w \in \mathfrak{M}(F)} \max _{1 \leq i \leq n} \frac{\left\|L_{i}^{(w)}(\boldsymbol{x})\right\|_{w}}{B_{i w}} .
$$

Let $\lambda_{1}^{\prime}, \ldots, \lambda_{n}^{\prime}$ be the successive minima of $H_{\boldsymbol{B}}(\boldsymbol{x})$. Writing $B_{q}=\prod_{i=1}^{n} B_{i q}(q \in \mathfrak{M}(E))$ we may infer from Proposition 7.1 that

$$
n^{-\frac{n}{2}} \prod_{q \in \mathfrak{M}(E)} \frac{\Delta_{q}}{B_{q}} \leq \lambda_{1}^{\prime} \ldots \lambda_{n}^{\prime} \leq 2^{\frac{n(n-1)}{2}} \prod_{q \in \mathfrak{M}(E)} \frac{\Delta_{q}}{B_{q}},
$$

where $\Delta_{q}=\left\|\operatorname{det}\left(L_{1}^{(q)}, \ldots, L_{n}^{(q)}\right)\right\|_{q}(q \in \mathfrak{M}(E))$.

We next compare the heights $H_{\boldsymbol{A}}(\boldsymbol{x})$ and $H_{\boldsymbol{B}}(\boldsymbol{x})$.

Again let $F$ be a finite extension of $E$, such that $\boldsymbol{x} \in F^{n}$. Let $w \in \mathfrak{M}(F)$ be a place lying 
above $q \in \mathfrak{M}(E)$, and suppose moreover that $q$ lies above $v \in \mathfrak{M}(K)$. Using the relation $d(w / v)=d(w / q) d(q / v)$ we obtain in view of (7.1), (7.2), (7.11), (7.12)

$$
\left\{\begin{array}{cl}
(1+\varepsilon)^{-d(w / v)} A_{i w} \leq B_{i w} \leq(1+\varepsilon)^{d(w / v)} A_{i w} & \text { for } w \in \mathfrak{M}(F) \text { lying } \\
& \text { above } v \in S \text { and } \\
& \text { for } i=1, \ldots, n \\
A_{i w}=B_{i w} & \text { for } w \in \mathfrak{M}(F) \text { not lying } \\
& \text { above some } v \in S \\
& \text { and for } i=1, \ldots, n .
\end{array}\right.
$$

On the other hand $H_{\boldsymbol{A}}(\boldsymbol{x})$ and $H_{\boldsymbol{B}}(\boldsymbol{x})$ involve the same linear forms. Thus by (7.15) the terms making up $H_{\boldsymbol{A}}(\boldsymbol{x})$ and $H_{\boldsymbol{B}}(\boldsymbol{x})$ satisfy

$$
\frac{\left\|L_{i}^{(w)}(\boldsymbol{x})\right\|_{w}}{A_{i w}}=\frac{\left\|L_{i}^{(w)}(\boldsymbol{x})\right\|_{w}}{B_{i w}}
$$

for $i=1, \ldots, n$ and for $w \in \mathfrak{M}(F)$ not lying above some $v \in S$. Moreover

$$
(1+\varepsilon)^{-d(w / v)} \frac{\left\|L_{i}^{(w)}(\boldsymbol{x})\right\|_{w}}{A_{i w}} \leq \frac{\left\|L_{i}^{(w)}(\boldsymbol{x})\right\|_{w}}{B_{i w}} \leq(1+\varepsilon)^{d(w / v)} \frac{\left\|L_{i}^{(w)}(\boldsymbol{x})\right\|_{w}}{A_{i w}}
$$

for $i=1, \ldots, n$ and for $w \in \mathfrak{M}(F)$ lying above $v \in S$. Writing $s$ for the cardinality of $S$ we may infer that for any $\boldsymbol{x} \in \overline{\mathbb{Q}}^{n}$

$$
(1+\varepsilon)^{-s} H_{\boldsymbol{A}}(\boldsymbol{x}) \leq H_{\boldsymbol{B}}(\boldsymbol{x}) \leq(1+\varepsilon)^{s} H_{\boldsymbol{A}}(\boldsymbol{x})
$$

and therefore

$$
(1+\varepsilon)^{-s} \lambda_{i} \leq \lambda_{i}^{\prime} \leq(1+\varepsilon)^{s} \lambda_{i} \quad(i=1, \ldots, n) .
$$

We now compare the quantities

$$
\prod_{q \in \mathfrak{M}(E)} \frac{\Delta_{q}}{B_{q}} \text { and } \prod_{v \in \mathfrak{M}(K)} \frac{\Delta_{v}}{A_{v}}
$$

For $v \in \mathfrak{M}(K)$ we obtain

$$
\prod_{q \in \mathfrak{M}(E), q \mid v} \Delta_{q}=\Delta_{v}
$$

and by (7.15)

$$
\begin{array}{rlrl}
(1+\varepsilon)^{-n} A_{v} & \leq \prod_{q \in \mathfrak{M}(E), q \mid v} B_{q} \leq(1+\varepsilon)^{n} A_{v} & & \text { for } v \in S \\
A_{v} & =\prod_{q \in \mathfrak{M}(E), q \mid v} B_{q} & \text { for } v \notin S .
\end{array}
$$


Altogether this implies

$$
(1+\varepsilon)^{-n s} \prod_{v \in \mathfrak{M}(K)} \frac{\Delta_{v}}{A_{v}} \leq \prod_{q \in \mathfrak{M}(E)} \frac{\Delta_{q}}{B_{v}} \leq(1+\varepsilon)^{n s} \prod_{v \in \mathfrak{M}(K)} \frac{\Delta_{v}}{A_{v}} .
$$

Combination of (7.14), (7.16), (7.17) yields

$$
(1+\varepsilon)^{-2 n s} n^{-n / 2} \prod_{v \in \mathfrak{M}(K)} \frac{\Delta_{v}}{A_{v}} \leq \lambda_{1} \ldots \lambda_{n} \leq(1+\varepsilon)^{2 n s} 2^{\frac{n(n-1)}{2}} \prod_{v \in \mathfrak{M}(K)} \frac{\Delta_{v}}{A_{v}} .
$$

Since $\varepsilon>0$ is arbitrary, the Corollary follows.

In (6.10), (6.11) we have defined the parallelepiped $\Pi(Q, \boldsymbol{c})$ which corresponds to the height $H_{Q, \boldsymbol{c}}(\boldsymbol{x})$. In complete analogy, we define the parallelepiped $\Pi(\boldsymbol{A})$ corresponding to the height $H_{\boldsymbol{A}}(\boldsymbol{x})$ as follows:

For a number field $F$ containing $K$ we write $\Pi_{F}(\boldsymbol{A})$ for the set of points $\boldsymbol{x} \in F^{n}$ satisfying

$$
\left\|L_{i}^{(w)}(\boldsymbol{x})\right\|_{w} \leq A_{i w} \quad(w \in \mathfrak{M}(F), i=1, \ldots, n),
$$

and we put

$$
\Pi(\boldsymbol{A})=\bigcup_{F \supset K} \Pi_{F}(\boldsymbol{A})
$$

where the union is over all finite extensions $F$ of $K$. Moreover, given $\lambda>0$, we define $\lambda * \Pi(\boldsymbol{A})$ in analogy with $(6.13)-(6.15)$.

We may introduce successive minima $\mu_{1}, \ldots, \mu_{n}$ of $\Pi(\boldsymbol{A})$ as follows. For $\mu>0$ we let $U_{\boldsymbol{A}}(\mu)$ be the $\overline{\mathbb{Q}}$ - vectorspace generated by the elements $\boldsymbol{x} \in \overline{\mathbb{Q}}^{n}$ with $\boldsymbol{x} \in \mu * \Pi(\boldsymbol{A})$. For $i=1, \ldots, n$ we then put

$$
\mu_{i}=\inf \left\{\mu \mid \operatorname{dim} U_{\boldsymbol{A}}(\mu) \geq i\right\} .
$$

The following Lemma indicates that there is no big difference between the space $V_{\boldsymbol{A}}(\lambda)$ defined via the height $H_{\boldsymbol{A}}(\boldsymbol{x})$ and the space $U_{\boldsymbol{A}}(\lambda)$ corresponding to the parallelepiped $\Pi(\boldsymbol{A})$.

Lemma 7.3 Let $\vartheta>0$ and $\lambda>0$. Then for any $\boldsymbol{x} \in \overline{\mathbb{Q}}^{n}$ with

$$
H_{\boldsymbol{A}}(\boldsymbol{x}) \leq \lambda
$$

there exists $\beta \in \overline{\mathbb{Q}}^{*}$ such that

$$
\beta \boldsymbol{x} \in(1+\vartheta) \lambda * \Pi(\boldsymbol{A}) .
$$

The proof is analogous to the proof of Lemma 6.2. It suffices to apply Lemma 6.3. We will not detail it her.

As a consequence of Lemma 7.3 we obtain 
Corollary 7.4 The successive minima $\mu_{1}, \ldots, \mu_{n}$ of $\Pi(\boldsymbol{A})$ coincide with the successive minima $\lambda_{1}, \ldots, \lambda_{n}$ of $H_{\boldsymbol{A}}(\boldsymbol{x})$.

Proof. Our definitions of $H_{\boldsymbol{A}}$ and $\Pi(\boldsymbol{A})$ imply that if $\boldsymbol{x} \in \lambda * \Pi(\boldsymbol{A})$ then $H_{\boldsymbol{A}}(\boldsymbol{x}) \leq \lambda$. Consequently, for $i=1, \ldots, n$ we get

$$
\lambda_{i} \leq \mu_{i}
$$

On the other hand, using Lemma 7.3 we see that any $\vartheta>0$ and for $i=1, \ldots, n$

$$
\mu_{i} \leq(1+\vartheta) \lambda_{i}
$$

The Corollary follows.

In view of Corollary 7.4 from now on we write $\lambda_{1}, \ldots, \lambda_{n}$ for the successive minima of $H_{\boldsymbol{A}}(\boldsymbol{x})$ as well as of $\Pi(\boldsymbol{A})$.

Corollary 7.5 Suppose $1 \leq i<n$ and that

$$
\lambda_{i}<\lambda_{i+1}
$$

Then there exists a subspace $T$ of $\overline{\mathbb{Q}}^{n}$ of dimension $i$ such that for each $\lambda$ with

$$
\lambda_{i}<\lambda<\lambda_{i+1}
$$

we have

$$
V_{\boldsymbol{A}}(\lambda)=U_{\boldsymbol{A}}(\lambda)=T
$$

Moreover $T$ is defined over $K$.

Proof. Our definitions of $\lambda * \Pi(\boldsymbol{A})$ and of $H_{\boldsymbol{A}}(\boldsymbol{x}) \leq \lambda$ imply at once

$$
U_{\boldsymbol{A}}(\lambda) \subseteq V_{\boldsymbol{A}}(\lambda)
$$

On the other hand by Lemma 7.3 we obtain for any $\varepsilon>0$

$$
V_{\boldsymbol{A}}(\lambda) \subseteq U_{\boldsymbol{A}}(\lambda(1+\varepsilon))
$$

To prove the relation $V_{\boldsymbol{A}}(\lambda)=U_{\boldsymbol{A}}(\lambda)$ it therefore suffices to prove that for any pair $\lambda, \tilde{\lambda}$ with

$$
\lambda_{i}<\lambda<\widetilde{\lambda}<\lambda_{i+1}
$$

we have

$$
U_{\boldsymbol{A}}(\lambda)=U_{\boldsymbol{A}}(\widetilde{\lambda}) .
$$


But this is plain from the definition of the successive minima and (7.23). As for the rationality of $V_{\boldsymbol{A}}(\lambda)$, by definition, $V_{\boldsymbol{A}}(\lambda)$ is generated by $M=\left\{\boldsymbol{x} \in \overline{\mathbb{Q}}^{n} \mid H_{\boldsymbol{A}}(\boldsymbol{x}) \leq \lambda\right\}$. In exactly the same way as in the proof of Lemma 4.1 we may show that for each $\sigma \in$ $\operatorname{Gal}(\overline{\mathbb{Q}} / K)$

$$
H_{\boldsymbol{A}}(\sigma \boldsymbol{x})=H_{\boldsymbol{A}}(\boldsymbol{x}) \quad \text { for each } \boldsymbol{x} \in \overline{\mathbb{Q}}^{n} .
$$

In particular $M$ satisfies the hypothesis of Lemma 4.2 with $F$ replaced by $K$. By that Lemma we may conclude that indeed $V_{\boldsymbol{A}}(\lambda)$ is defined over $K$.

We finally apply Corollary 7.2 to the parallelepiped $\Pi(Q, \boldsymbol{c})$ given by $(6.10),(6.11)$ with forms $L_{i}^{(v)}$ and with parameters $c_{i v}$ satisfying (6.1) - (6.8). So in our special situation the parameters $A_{i v}$ in Corollary 7.2 become $Q^{c_{i v}}$. Accordingly, the successive minima $\lambda_{i}=\lambda_{i}(Q)$ will depend upon the parameter $Q$. Combination of Corollary 7.2 and of (6.4), (6.5) yields

Corollary 7.6 For each $Q \geq 1$ the successive minima of $\Pi(Q, \boldsymbol{c})$ satisfy

$$
n^{-\frac{n}{2}} \leq \lambda_{1}(Q) \ldots \lambda_{n}(Q) \leq 2^{\frac{n(n-1)}{2}} .
$$

\section{A Gap Principle}

Our goal in this section is to prove

Lemma 8.1 Let $\Pi(Q, \boldsymbol{c})$ be the parallelepiped in $\overline{\mathbb{Q}}^{n}$ defined by (6.10), (6.11) with forms $L_{i}^{(v)}$ and tuples $\boldsymbol{c}=\left(c_{i v}\right)(v \in \mathfrak{M}(K), i=1, \ldots, n)$ as in $(6.1)$ - (6.8).

Suppose $0<\delta \leq 1$ and $B>1$, and let $Q_{0}$ be a parameter satisfying

$$
Q_{0} \geq n^{1 / \delta}
$$

Then there are proper linear subspaces $T_{1}, \ldots, T_{t}$ of $\overline{\mathbb{Q}}^{n}$ where

$$
t \leq 1+\frac{5}{\delta} \log B
$$

with the following property:

For each $Q$ with

$$
Q_{0}<Q \leq Q_{0}^{B}
$$

there exists $i$ with $1 \leq i \leq t$ such that

$$
Q^{-\delta} * \Pi(Q, \boldsymbol{c}) \subset T_{i}
$$

We will derive Lemma 8.1 from the following two auxiliary Lemmata. 
Lemma 8.2 Suppose

$$
\delta>0, Q_{1}>1, Q_{1}<Q \leq Q_{1}^{1+\frac{2}{5} \delta}
$$

Let $\varepsilon>0$.

Then for any $\boldsymbol{x} \in Q^{-\delta} * \Pi(Q, \boldsymbol{c})$ there exists $\beta \in \overline{\mathbb{Q}}^{*}$ such that

$$
\beta \boldsymbol{x} \in(1+\varepsilon) Q_{1}^{-\frac{3}{5} \delta} * \Pi\left(Q_{1}, \boldsymbol{c}\right) .
$$

Proof. Let $\boldsymbol{x} \in Q^{-\delta} * \Pi(Q, \boldsymbol{c})$. Let $F$ be a finite extension of $K$ such that $\boldsymbol{x} \in F^{n}$. Then by $(6.13),(6.14)$ we have

$$
\left\{\begin{array}{cl}
\left\|L_{i}^{(w)}(\boldsymbol{x})\right\|_{w} \leq Q^{c_{i w}} & \left(w \in \mathfrak{M}(F), w \nmid v_{0}, i=1, \ldots, n\right) \\
\left\|x_{i}\right\|_{w} \leq Q^{-\delta d\left(w / v_{0}\right)} & \left(w \in \mathfrak{M}(F), w \mid v_{0}, i=1, \ldots, n\right) .
\end{array}\right.
$$

For $w \in \mathfrak{M}(F)$ we write

$$
c_{w}=\max \left\{c_{1 w}, \ldots, c_{n w}\right\} .
$$

Notice that by (6.5)

$$
\sum_{w \in \mathfrak{M}(F)} c_{w} \leq 1
$$

(8.5) yields for $w \in \mathfrak{M}(F), i=1, \ldots, n$

$$
Q^{c_{i w}}=Q^{c_{i w}-c_{w}} Q^{c_{w}} \leq Q_{1}^{c_{i w}-c_{w}} Q_{1}^{c_{w}}\left(\frac{Q}{Q_{1}}\right)^{c_{w}}=Q_{1}^{c_{i w}}\left(\frac{Q}{Q_{1}}\right)^{c_{w}}
$$

whence by (8.7)

$$
\left\{\begin{array}{cl}
\left\|L_{i}^{(w)}(\boldsymbol{x})\right\|_{w} \leq Q_{1}^{c_{i w}}\left(\frac{Q}{Q_{1}}\right)^{c_{w}} & \left(w \in \mathfrak{M}(F), w \nmid v_{0}, i=1, \ldots, n\right) \\
\left\|x_{i}\right\|_{w} \leq Q_{1}^{-\delta d\left(w / v_{0}\right)} & \left(w \in \mathfrak{M}(F), w \mid v_{0}, i=1, \ldots, n\right) .
\end{array}\right.
$$

We want to apply Lemma 6.3 with

$$
A_{w}= \begin{cases}\left(Q / Q_{1}\right)^{-c_{w}} & \left(w \in \mathfrak{M}(F), w \nmid v_{0}\right) \\ \left\{(1+\varepsilon)\left(Q / Q_{1}\right)\right\}^{d\left(w / v_{0}\right)} & \left(w \in \mathfrak{M}(F), w \mid v_{0}\right) .\end{cases}
$$

Let $R$ be the set of places $w \in \mathfrak{M}(F)$ with $c_{w} \neq 0$. By (6.6), (6.7), $R$ is finite. Thus for all but finitely many $w \in \mathfrak{M}(F)$ we have $A_{w}=1$. Moreover by (6.7), (6.12) we get

$$
c_{w}=0 \text { for } w \mid v_{0} \text {. }
$$

Combination of (8.11), (8.12), (8.8) yields

$$
\prod_{w \in \mathfrak{M}(F)} A_{w}=\left(Q / Q_{1}\right)^{-\left(\sum_{w \in \mathfrak{M}(F)} c_{w}\right)} \cdot(1+\varepsilon) \frac{Q}{Q_{1}} \geq(1+\varepsilon)>1 .
$$


Thus the hypotheses of Lemma 6.3 are satisfied.

Accordingly there exist a finite extension $E$ of $F$ and $\beta \in E^{*}$ with

$$
\|\beta\|_{u} \leq A_{w}^{d(u / w)} \quad(u \in \mathfrak{M}(E), u \mid w, w \in \mathfrak{M}(F)) .
$$

Writing for $u \in \mathfrak{M}(E) \quad c_{u}=\max \left\{c_{1 u}, \ldots, c_{n u}\right\}$, this means that

$$
\begin{aligned}
\|\beta\|_{u} & \leq\left(\frac{Q}{Q_{1}}\right)^{-c_{u}} & & \left(u \in \mathfrak{M}(E), u \nmid v_{0}\right) \\
\|\beta\|_{u} & \leq\left((1+\varepsilon) \frac{Q}{Q_{1}}\right)^{d\left(u / v_{0}\right)} & & \left(u \in \mathfrak{M}(E), u \mid v_{0}\right) .
\end{aligned}
$$

Using the analogue of (8.10) with $E$ instead of $F$ we obtain in view of

$$
c_{i u}=d(u / w) c_{i w}, \quad c_{u}=d(u / w) c_{w}(u \in \mathfrak{M}(E), u \mid w, w \in \mathfrak{M}(F))
$$

and by (8.5) the relations

$$
\begin{aligned}
&\left\|L_{i}^{(u)}(\beta \boldsymbol{x})\right\|_{u} \leq\left(Q_{1}^{c_{i w}}\left(\frac{Q}{Q_{1}}\right)^{c_{w}}\right)^{d(u / w)}\left(\frac{Q}{Q_{1}}\right)^{-c_{u}}=Q_{1}^{c_{i u}} \\
&\left(u \in \mathfrak{M}(E), u \nmid v_{0}, i=1, \ldots, n\right), \\
&\left\|\beta x_{i}\right\|_{u} \leq Q_{1}^{-\delta d\left(w / v_{0}\right) d(u / w)}\left((1+\varepsilon) \frac{Q}{Q_{1}}\right)^{d\left(u / v_{0}\right)}=\left((1+\varepsilon) \frac{Q}{Q_{1}^{1+\frac{2}{5} \delta}} Q_{1}^{-\frac{3}{5} \delta}\right)^{d\left(u / v_{0}\right)} \\
& \leq\left((1+\varepsilon) Q_{1}^{-\frac{3}{5} \delta}\right)^{d\left(u / v_{0}\right)}\left(u \in \mathfrak{M}(E), u \mid v_{0}, i=1, \ldots, n\right) .
\end{aligned}
$$

But this is the assertion in (8.6).

\section{Lemma 8.3 Suppose}

$$
\delta>0, Q_{1} \geq n^{1 / \delta} .
$$

Then there is a proper linear subspace $T$ of $\overline{\mathbb{Q}}^{n}$ with the following property:

For every $Q$ with

$$
Q_{1} \leq Q \leq Q_{1}^{1+\frac{2}{5} \delta}
$$

we have

$$
Q^{-\delta} * \Pi(Q, \boldsymbol{c}) \subset T
$$

Proof. By (8.13) there exists $\varepsilon>0$ such that

$$
(1+\varepsilon) Q_{1}^{-\frac{3}{5} \delta} \leq(1+\varepsilon) n^{-\frac{3}{5}}<n^{-\frac{1}{2}} .
$$

Let $T$ be the subspace of $\overline{\mathbb{Q}}^{n}$ generated by the vectors $\boldsymbol{y} \in \overline{\mathbb{Q}}^{n}$ with

$$
\boldsymbol{y} \in(1+\varepsilon) Q_{1}^{-\frac{3}{5} \delta} * \Pi\left(Q_{1}, \boldsymbol{c}\right) .
$$


By Corollary 7.6 the $n$-th minimum $\lambda_{n}$ of $\Pi\left(Q_{1}, \boldsymbol{c}\right)$ satisfies

$$
\lambda_{n} \geq n^{-\frac{1}{2}} .
$$

By Corollary 7.5, (8.16) and (8.17), $T$ has dimension $<n$.

On the other hand, in view of Lemma 8.2 for any $\boldsymbol{x} \in Q^{-\delta} * \Pi(Q, \boldsymbol{c})$ with $Q$ as in (8.14) we can find a nonzero multiple $\beta \boldsymbol{x}$ such that $\beta \boldsymbol{x} \in(1+\varepsilon) Q_{1}^{-\frac{3}{5} \delta} * \Pi\left(Q_{1}, \boldsymbol{c}\right)$. Therefore $\beta \boldsymbol{x} \in T$, whence $\boldsymbol{x} \in T$. This proves (8.15).

The assertion of Lemma 8.1 now follows easily. The interval $\left(Q_{0}, Q_{0}^{B}\right]$ in (8.3) may be covered by

$$
\leq 1+\frac{\log B}{\log \left(1+\frac{2}{5} \delta\right)} \leq 1+\frac{5}{\delta} \log B
$$

intervals of type (8.14).

By Lemma 8.3, the collection of sets $Q^{-\delta} * \Pi(Q, \boldsymbol{c})$ where $Q$ runs through a fixed interval (8.14) may be covered by a single proper subspace $T$ of $\overline{\mathbb{Q}}^{n}$. Therefore, to cover the whole collection of sets $Q^{-\delta} * \Pi(Q, \boldsymbol{c})$ with $Q$ as in (8.3),

$$
t \leq 1+\frac{5}{\delta} \log B
$$

subspaces will suffice and Lemma 8.1 follows.

\section{Davenport's Lemma}

In this section we adjust Davenport's Lemma (Schmidt [14], Lemma 6.1) to our current situation. Actually, following Evertse [4] we prove a stronger version which is crucial to guarantee that the bound (2.16) in Theorem 2.1 is not doubly exponential in $n$.

Given a number field $E$, we introduce for $u \in \mathfrak{M}(E)$ the quantities

$$
s(u)=\left\{\begin{array}{cl}
{\left[E_{u}: \mathbb{R}\right] /[E: \mathbb{Q}]} & \text { for } u \in \mathfrak{M}_{\infty}(E) \\
0 & \text { for } u \in \mathfrak{M}_{0}(E) .
\end{array}\right.
$$

This notation will be used throughout the remainder of the paper. It has the advantage that at several instances we do not have to make a distinction between estimates for archimedean absolute values and for nonarchimedean absolute values.

Lemma 9.1 Let $t \geq 2$. Suppose $\vartheta_{1}, \ldots, \vartheta_{t-1} \in \overline{\mathbb{Q}}$. Suppose $\varepsilon>0$. Let $v_{0} \in \mathfrak{M}_{0}(K)$ be as in (6.12).

Then there exists a finite extension $E$ of $K$ and a vector $\gamma=\left(\gamma_{1}, \ldots, \gamma_{t}\right) \in E^{t}$ with the following properties:

$$
\vartheta_{1}, \ldots, \vartheta_{t-1} \in E \text {. }
$$




$$
\left\|\gamma_{i}\right\|_{u} \leq t^{-s(u)} \quad\left(u \in \mathfrak{M}_{\infty}(E), 1 \leq i \leq t\right)
$$

$$
\begin{gathered}
\left\|\gamma_{i}-\vartheta_{i} \gamma_{t}\right\|_{u} \leq 1, \quad\left\|\gamma_{t}\right\|_{u} \leq\left((1+\varepsilon)^{t} 2^{t^{2}}\right)^{d\left(u / v_{0}\right)} \quad\left(u \in \mathfrak{M}(E), u \mid v_{0}, 1 \leq i<t\right) \\
\left\|\gamma_{i}\right\|_{u} \leq 1 \quad\left(u \in \mathfrak{M}_{0}(E) \backslash\left\{v_{0}\right\}, 1 \leq i \leq t\right) \\
\gamma_{t} \neq 0 .
\end{gathered}
$$

Proof. Let $F$ be a finite extension of $K$ containing $\vartheta_{1}, \ldots, \vartheta_{t-1}$. For $w \in \mathfrak{M}(F)$ we define linear forms $l_{1}^{(w)}, \ldots, l_{t}^{(w)}$ in $X_{1}, \ldots, X_{t}$ :

For $w \in \mathfrak{M}(F)$ with $w \mid v_{0}$ we put

$$
l_{i}^{(w)}=X_{i}-\vartheta_{i} X_{t} \quad(i=1, \ldots, t-1), \quad l_{t}^{(w)}=X_{t},
$$

whereas for $w \in \mathfrak{M}(F), w \nmid v_{0}$ we put

$$
l_{i}^{(w)}=X_{i} \quad(i=1, \ldots, t) .
$$

Moreover we define the tuple $\boldsymbol{A}=\left(A_{i w} \mid w \in \mathfrak{M}(F), 1 \leq i \leq t\right)$ by

$$
\begin{gathered}
A_{i w}=t^{-s(w)} \quad\left(w \in \mathfrak{M}_{\infty}(F), 1 \leq i \leq t\right) \\
A_{i w}=1 \quad\left(w \in \mathfrak{M}(F), w \nmid v_{0}, 1 \leq i \leq t\right) \\
A_{i w}=1(1 \leq i \leq t-1), A_{t w}=\left(t^{t}(1+\varepsilon)^{t} 2^{\frac{t(t-1)}{2}}\right)^{d\left(w / v_{0}\right)}\left(w \in \mathfrak{M}(F), w \mid v_{0}\right) .
\end{gathered}
$$

Let $\Pi_{F}(\boldsymbol{A}, \boldsymbol{l})$ be the parallelepiped in $F^{t}$ given by the inequalities

$$
\left\|l_{i}^{(w)}(\boldsymbol{x})\right\|_{w} \leq A_{i w} \quad(w \in \mathfrak{M}(F), i=1, \ldots, t)
$$

and write $\Pi(\boldsymbol{A}, \boldsymbol{l})$ for its algebraic closure.

For $\lambda>0$ we define $\lambda * \Pi_{F}(\boldsymbol{A}, \boldsymbol{l})$ by

$$
\begin{array}{ll}
\left\|l_{i}^{(w)}(\boldsymbol{x})\right\|_{w} \leq A_{i w} & \left(w \in \mathfrak{M}(F), w \nmid v_{0}, i=1, \ldots, t\right) \\
\left\|l_{i}^{(w)}(\boldsymbol{x})\right\|_{w} \leq A_{i w} \lambda^{d\left(w / v_{0}\right)} & \left(w \in \mathfrak{M}(F), w \mid v_{0}, i=1, \ldots, t\right) .
\end{array}
$$

$\lambda * \Pi(\boldsymbol{A}, \boldsymbol{l})$ will denote the natural extension of $(9.12)$ to $\overline{\mathbb{Q}}^{t}$. By $(9.7),(9.8)$ we get

$$
\prod_{w \in \mathfrak{M}(F)}\left\|\operatorname{det}\left(l_{1}^{(w)}, \ldots, l_{t}^{(w)}\right)\right\|_{w}=1
$$


(9.9) - (9.11) imply that

$$
\prod_{w \in \mathfrak{M}(F)} \prod_{i=1}^{t} A_{i w}=(1+\varepsilon)^{t} 2^{\frac{t(t-1)}{2}} .
$$

Let $\lambda_{1}, \ldots, \lambda_{t}$ be the successive minima of $\Pi(\boldsymbol{A}, \boldsymbol{l})$. By Proposition 7.1 and Corollaries 7.2 and 7.4 we obtain

$$
\lambda_{1} \ldots \lambda_{t} \leq(1+\varepsilon)^{-t}
$$

and therefore in particular

$$
\lambda_{1} \leq(1+\varepsilon)^{-1} .
$$

We may conclude that there are a finite extension $E$ of $F$ and a point $\boldsymbol{\gamma}=\left(\gamma_{1}, \ldots, \gamma_{t}\right) \neq \mathbf{0}$ in $E^{n}$ with

$$
\begin{array}{ll}
\left\|l_{i}^{(u)}(\gamma)\right\|_{u} \leq A_{i u} & \left(u \in \mathfrak{M}(E), u \nmid v_{0}, i=1, \ldots, t\right) \\
\left\|l_{i}^{(u)}(\gamma)\right\|_{u} \leq A_{i u}\left((1+\varepsilon) \lambda_{1}\right)^{d\left(u / v_{0}\right)} \leq A_{i u} & \left(u \in \mathfrak{M}(E), u \mid v_{0}, i=1, \ldots, t\right) .
\end{array}
$$

Combination of (9.7) - (9.11), (9.13) yields assertions (9.3) - (9.5).

We still have to prove (9.6).

Assume $\gamma_{t}=0$. Then by (9.3) - (9.5) we would obtain

$$
\prod_{u \in \mathfrak{M}(E)}\left\|\gamma_{i}\right\|_{u} \leq t^{-1}<1 \quad(1 \leq i \leq t-1)
$$

and therefore $\gamma_{1}=\ldots=\gamma_{t-1}=0$. Since $\boldsymbol{\gamma} \neq \mathbf{0}$, this would be a contradiction, and (9.6) follows as well.

We now consider our forms $L_{i}^{(v)}$ and our tuples $\boldsymbol{c}=\left(c_{i v}\right)(v \in \mathfrak{M}(K), i=1, \ldots, n)$ from (6.1) - (6.8) and we let $\Pi(Q, \boldsymbol{c})$ be the parallelepiped in $\overline{\mathbb{Q}}^{n}$ defined in (6.10), (6.11).

Let $\lambda_{1}, \ldots, \lambda_{n}$ be the successive minima of $\Pi(Q, \boldsymbol{c})$ and define the integers

$$
1 \leq r_{1}<r_{2}<\ldots<r_{s}=n
$$

by

$$
\lambda_{1}=\ldots=\lambda_{r_{1}}<\lambda_{r_{1}+1}=\ldots=\lambda_{r_{2}}<\ldots<\lambda_{r_{s-1}+1}=\ldots=\lambda_{n}
$$

Let $\varepsilon$ be a positive number, small enough such that

$$
\lambda_{r_{t}}(1+\varepsilon)^{2}<\lambda_{r_{t+1}} \quad(t=1, \ldots, s-1) .
$$

Moreover let $\boldsymbol{g}_{1}, \ldots, \boldsymbol{g}_{n}$ be linearly independent points in $\overline{\mathbb{Q}}^{n}$ with

$$
\boldsymbol{g}_{j} \in(1+\varepsilon) \lambda_{j} * \Pi(Q, \boldsymbol{c}) \quad(1 \leq j \leq n) .
$$


Write $\boldsymbol{g}_{j}=\left(g_{j 1}, \ldots, g_{j n}\right)$ and let $F$ be a finite extension of $K$ with $\boldsymbol{g}_{1}, \ldots, \boldsymbol{g}_{n} \in F^{n}$. By (6.13), (6.14) we may detail (9.16) as

$$
\begin{array}{r}
\left\|L_{i}^{(w)}\left(\boldsymbol{g}_{j}\right)\right\|_{w} \leq Q^{c_{i w}} \quad\left(w \in \mathfrak{M}(F), w \nmid v_{0}, 1 \leq i, j \leq n\right) \\
\left\|L_{i}^{(w)}\left(\boldsymbol{g}_{j}\right)\right\|_{w}=\left\|g_{j i}\right\|_{w} \leq \quad \begin{array}{r}
\left((1+\varepsilon) \lambda_{j}\right)^{d\left(w / v_{0}\right)} \\
\left(w \in \mathfrak{M}(F), w \mid v_{0}, 1 \leq i, j \leq n\right) .
\end{array}
\end{array}
$$

Lemma 9.2 (Davenport's Lemma).

There exists a finite extension $E$ of $K$ and there exists a permutation $\pi$ of $\{1, \ldots, n\}$ with the following properties:

We can find vectors $\boldsymbol{h}_{1}=\left(h_{11}, \ldots, h_{1 n}\right), \ldots, \boldsymbol{h}_{n}=\left(h_{n 1}, \ldots, h_{n n}\right)$ in $E^{n}$ satisfying

$$
\begin{aligned}
& \text { for } j=1, \ldots, n \text { the set }\left\{\boldsymbol{h}_{1}, \ldots, \boldsymbol{h}_{j}\right\} \text { spans the same } \overline{\mathbb{Q}} \text {-vector space as } \\
& \left\{\boldsymbol{g}_{1}, \ldots, \boldsymbol{g}_{j}\right\}
\end{aligned}
$$

and

$$
\begin{aligned}
\left\|L_{i}^{(u)}\left(\boldsymbol{h}_{j}\right)\right\|_{u} & \leq Q^{c_{i u}} \quad\left(u \in \mathfrak{M}(E), u \nmid v_{0}, 1 \leq i, j \leq n\right) \\
\left\|L_{\pi(i)}^{(u)}\left(\boldsymbol{h}_{j}\right)\right\|_{u}=\left\|h_{j \pi(i)}\right\|_{u} \leq \quad\left((1+\varepsilon)^{n+1} 2^{n^{2}} \min \left\{\lambda_{i}, \lambda_{j}\right\}\right)^{d\left(u / v_{0}\right)} & \left(u \in \mathfrak{M}(E), u \mid v_{0}, 1 \leq i, j \leq n\right) .
\end{aligned}
$$

Proof. We first determine the permutation $\pi$. For $t=1, \ldots, s$, let $V_{r_{t}}$ be the $\overline{\mathbb{Q}}$-vector space spanned by $\boldsymbol{g}_{1}, \ldots, \boldsymbol{g}_{r_{t}}$. By (9.15), (9.16) and Corollary 7.5, for each $t$ the space $V_{r_{t}}$ is defined over $K$. Consequently it has a basis $\boldsymbol{y}_{1}, \ldots, \boldsymbol{y}_{r_{t}}$ with $\boldsymbol{y}_{j} \in K^{n}$ $\left(1 \leq j \leq r_{t}\right)$. In fact we may pick points $\boldsymbol{y}_{1}, \ldots, \boldsymbol{y}_{n}$ such that

$$
\boldsymbol{y}_{1}, \ldots, \boldsymbol{y}_{n} \text { are linearly independent }
$$

$$
\boldsymbol{y}_{1}, \ldots, \boldsymbol{y}_{n} \in K^{n}
$$

For $i=1, \ldots, n$, let $V_{i}$ be the subspace of $\overline{\mathbb{Q}}^{n}$ generated by $\boldsymbol{y}_{1}, \ldots, \boldsymbol{y}_{i}$. For a point $\boldsymbol{y}=\left(y_{1}, \ldots, y_{n}\right) \in V_{i}$ we obtain $n-i$ linearly independent linear relations between its components. Since $V_{i}$ has a basis in $K^{n}$, these relations may be taken such as to have coefficients in $K$.

In particular, on $V_{n-1}$ there is a nontrivial relation

$$
a_{1} y_{1}+\ldots+a_{n} y_{n}=0 \quad\left(\boldsymbol{y} \in V_{n-1}\right)
$$


with $a_{1}, \ldots, a_{n} \in K$.

Let $v_{0} \in \mathfrak{M}_{1} \subset \mathfrak{M}_{0}(K)$ be the distinguished place in (6.12). After reordering the variables, if necessary, we may suppose that in (9.24) the coefficient $a_{n}$ satisfies

$$
\left\|a_{n}\right\|_{v_{0}}=\max \left\{\left\|a_{1}\right\|_{v_{0}}, \ldots,\left\|a_{n}\right\|_{v_{0}}\right\} .
$$

Dividing by $a_{n}$, we may rewrite (9.24) as

$$
a_{1}^{\prime} y_{1}+\ldots+a_{n-1}^{\prime} y_{n-1}=y_{n} \quad\left(\boldsymbol{y} \in V_{n-1}\right)
$$

with $a_{1}^{\prime}, \ldots, a_{n-1}^{\prime} \in K$ and

$$
\left\|a_{i}^{\prime}\right\|_{v_{0}} \leq 1 \quad(i=1, \ldots, n-1) .
$$

Points $\boldsymbol{y} \in V_{n-2}$, apart from (9.26) will satisfy a second relation, independent of (9.26). Indeed we may find such a relation of type

$$
b_{1} y_{1}+\ldots+b_{n-1} y_{n-1}=0 \quad\left(\boldsymbol{y} \in V_{n-1}\right) .
$$

Again we may reorder the variables such that in (9.27)

$$
\left\|b_{n-1}\right\|_{v_{0}}=\max \left\{\left\|b_{1}\right\|_{v_{0}}, \ldots,\left\|b_{n-1}\right\|_{v_{0}}\right\} .
$$

So (9.27) will be equivalent to a relation of the shape

$$
b_{1}^{\prime} y_{1}+\ldots+b_{n-2}^{\prime} y_{n-2}=y_{n-1} \quad\left(\boldsymbol{y} \in V_{n-2}\right)
$$

with $b_{1}^{\prime}, \ldots, b_{n-2}^{\prime} \in K$ and

$$
\left\|b_{i}^{\prime}\right\|_{v_{0}} \leq 1 \quad(i=1, \ldots, n-2) .
$$

Now for points $\boldsymbol{y} \in V_{n-2}$, we may substitute (9.28) into (9.25), such that on $V_{n-2}$ relation (9.25) may be rewritten as

$$
a_{1}^{\prime \prime} y_{1}+\ldots+a_{n-2}^{\prime \prime} y_{n-2}=y_{n}
$$

with $a_{1}^{\prime \prime}, \ldots, a_{n-2}^{\prime \prime} \in K$ and

$$
\left\|a_{i}^{\prime \prime}\right\|_{v_{0}} \leq 1 \quad(i=1, \ldots, n-2) .
$$

(To guarantee (9.31) it is crucial that the distinguished place $v_{0}$ is non-archimedean.) We may continue in this manner. The final outcome is as follows. After a suitable permutation of the variables, for each $i(i=1, \ldots, n)$ the points $\boldsymbol{y} \in V_{i}$ will satisfy $n-i$ linearly independent relations of the following type

$$
y_{k}=\sum_{j=1}^{i} a_{k j}^{(i)} y_{j} \quad \text { for } k=i+1, \ldots, n
$$


with coefficients $a_{k j}^{(i)} \in K \quad(i+1 \leq k \leq n, 1 \leq j \leq i)$ having

$$
\left\|a_{k j}^{(i)}\right\|_{v_{0}} \leq 1 \quad(i+1 \leq k \leq n, 1 \leq j \leq 1)
$$

This finishes the construction of the permutation $\pi$. For simplicity of notation, from now on we assume our permutation to be the identity.

Before we start the construction of the points $\boldsymbol{h}_{j}$, we derive a very simple consequence from (9.32). Suppose $\boldsymbol{z}_{1}=\left(z_{11}, \ldots, z_{1 n}\right), \ldots, \boldsymbol{z}_{i}=\left(z_{i 1}, \ldots, z_{i n}\right)$ is a basis of $V_{i}$, then in the matrix

$$
\left(\begin{array}{c}
z_{11}, \ldots, z_{1 n} \\
z_{i 1}, \ldots, z_{i n}
\end{array}\right)
$$

the first $i$ columns are linearly independent. We may conclude that for any point $\boldsymbol{y}=\left(y_{1}, \ldots, y_{i}, y_{i+1}, \ldots, y_{n}\right) \in \overline{\mathbb{Q}}^{n}$ we can find elements $\vartheta_{1}, \ldots, \vartheta_{i}$ such that

$$
y_{j}=\vartheta_{1} z_{1 j}+\ldots+\vartheta_{i} z_{i j} \text { for } j=1, \ldots, i .
$$

We proceed to construct the vectors $\boldsymbol{h}_{1}, \ldots, \boldsymbol{h}_{n}$. The construction will be by induction on $t$ with $1 \leq t \leq s$ as the procedure to find $\boldsymbol{h}_{q}$ with $r_{t-1}+1 \leq q \leq r_{t}$ will follow the same pattern.

We start with $t=1$ taking $\boldsymbol{h}_{1}=\boldsymbol{g}_{1}, \ldots, \boldsymbol{h}_{r_{1}}=\boldsymbol{g}_{r_{1}}$. With this choice, by (9.14), (9.17), (9.18) relations (9.19), (9.20) are satisfied for $j=1, \ldots, r_{1}$ and for $i=1, \ldots, n$.

Now suppose that $1<t \leq s$ and that $\boldsymbol{h}_{1}, \ldots, \boldsymbol{h}_{r_{t-1}}$ have already been constructed such that $(9.19),(9.20)$ are true for $j=1, \ldots, r_{t-1}$ and for $i=1, \ldots, n$. Our vectors $\boldsymbol{h}_{r_{t-1}+1}, \ldots, \boldsymbol{h}_{r_{t}}$ will be of the shape

$$
\boldsymbol{h}_{q}=\gamma_{q q} \boldsymbol{g}_{q}+\sum_{j=1}^{r_{t-1}} \gamma_{q j} \boldsymbol{h}_{j} \quad \text { for } q=r_{t-1}+1, \ldots, r_{t}
$$

with coefficients $\gamma_{q q}, \gamma_{q j} \in \overline{\mathbb{Q}}, \gamma_{q q} \neq 0$ yet to be determined.

Suppose for the moment that for $q$ with $r_{t-1}+1 \leq q \leq r_{t}$ we have found suitable coefficients $\gamma_{q q}, \gamma_{q 1}, \ldots, \gamma_{q r_{t-1}}$ with $\gamma_{q q} \neq 0$. Then by the induction hypothesis it follows at once that assertion (9.19) will be true for $j=1, \ldots, r_{t}$.

So in the sequel we have only to worry about (9.20). We fix $q$ with $r_{t-1}+1 \leq q \leq r_{t}$ and we proceed to construct the point $\boldsymbol{h}_{q}$. To avoid heavy notation we write instead of (9.35)

$$
\boldsymbol{h}_{q}=\boldsymbol{h}=\gamma_{q} \boldsymbol{g}_{q}+\sum_{j=1}^{r_{t-1}} \gamma_{j} \boldsymbol{h}_{j}
$$

Here $\boldsymbol{h}=\left(h_{1}, \ldots, h_{n}\right)$. At the end of our construction we will return to double indices, i.e.,

$$
\boldsymbol{h}=\left(h_{1}, \ldots, h_{n}\right)=\left(h_{q 1}, \ldots, h_{q n}\right) .
$$


Relation (9.36) for the $i$-th component reads as

$$
h_{i}=\gamma_{q} g_{q i}+\sum_{j=1}^{r_{t-1}} \gamma_{j} h_{j i} \quad(i=1, \ldots, n) .
$$

We will first treat (9.38). We distinguish the ranges $1 \leq i \leq r_{t-1}$ and $r_{t-1}+1 \leq i \leq n$. We begin with the range $1 \leq i \leq r_{t-1}$. By the induction hypothesis $\boldsymbol{h}_{1}, \ldots, \boldsymbol{h}_{r_{t-1}}$ form a basis of $V_{r_{t-1}}$. Thus by (9.34) for $i=1, \ldots, r_{t-1}$ we obtain relations

$$
g_{q i}=\vartheta_{1} h_{1 i}+\ldots+\vartheta_{r_{t-1}} h_{r_{t-1}, i}
$$

with certain coefficients $\vartheta_{1}, \ldots, \vartheta_{r_{t-1}}$. Substituting (9.39) into (9.38) we get

$$
h_{i}=\sum_{j=1}^{r_{t-1}}\left(\gamma_{q} \vartheta_{j}+\gamma_{j}\right) h_{j i} \quad\left(i=1, \ldots, r_{t-1}\right) .
$$

We next deal with the range $r_{t-1}+1 \leq i \leq r_{t}$. By the induction hypothesis $\boldsymbol{h}_{1}, \ldots, \boldsymbol{h}_{r_{t-1}}$ lie in $V_{r_{t-1}}$. However, on $V_{r_{t-1}}$ we have relations (9.32). So there exist for $i=r_{t-1}+1, \ldots, n$ elements $a_{i 1}, \ldots, a_{i, r_{t-1}} \in K$ with

$$
\left\|a_{i k}\right\|_{v_{0}} \leq 1 \quad\left(1 \leq k \leq r_{t-1}\right)
$$

such that

$$
h_{j i}=\sum_{k=1}^{r_{t-1}} a_{i k} h_{j k} \quad\left(1 \leq j \leq r_{t-1}\right) .
$$

Substituting (9.42) into (9.38) and interchanging the order of summation we obtain

$$
h_{i}=\gamma_{q} g_{q i}+\sum_{k=1}^{r_{t-1}} a_{i k} \sum_{j=1}^{r_{t-1}} \gamma_{j} h_{j k} \quad\left(r_{t-1}+1 \leq i \leq n\right) .
$$

Notice that in (9.43) the inner sum by (9.38) equals

$$
\sum_{j=1}^{r_{t-1}} \gamma_{j} h_{j k}=-\gamma_{q} g_{q k}+h_{k}
$$

Substituting (9.44) into (9.43) we finally get

$$
h_{i}=\gamma_{q} g_{q i}+\sum_{k=1}^{r_{t-1}} a_{i k}\left(h_{k}-\gamma_{q} g_{q k}\right),
$$

and this is for $r_{t-1}+1 \leq i \leq n$.

So the components $h_{i}$ of our vector $\boldsymbol{h}$ to be constructed satisfy (9.40) and (9.45). 
We now choose the numbers $\gamma_{q}, \gamma_{1}, \ldots, \gamma_{r_{t-1}}$ in (9.38).

Let $F$ be a finite extension of $K$ such that $\boldsymbol{h}_{1}, \ldots, \boldsymbol{h}_{r_{t-1}}, \boldsymbol{g}_{q} \in F^{n}$. Then the elements $\vartheta_{1}, \ldots, \vartheta_{r_{t-1}}$ in (9.39) will lie in $F$ as well. We now apply Lemma 9.1 with $t$ replaced by $r_{t-1}+1$. Accordingly, given $\vartheta_{1}, \ldots, \vartheta_{r_{t-1}}$ we can find a finite extension $E$ of $F$ and elements $\gamma_{1}, \ldots, \gamma_{r_{t-1}}, \gamma_{q}$ in $E$ such that

$$
\left\{\begin{array}{l}
\left\|\gamma_{j}\right\|_{u} \leq\left(r_{t-1}+1\right)^{-s(u)} \quad\left(u \in \mathfrak{M}(E), u \nmid v_{0}, j=1, \ldots, r_{t-1} \text { and } j=q\right) \\
\left\|\gamma_{j}+\vartheta_{j} \gamma_{q}\right\|_{u} \leq 1 \quad\left(u \in \mathfrak{M}(E), u \mid v_{0}, j=1, \ldots, r_{t-1}\right) \\
\left\|\gamma_{q}\right\|_{u} \leq\left((1+\varepsilon)^{r_{t-1}+1} 2^{\left(r_{t-1}+1\right)^{2}}\right)^{d\left(u / v_{0}\right)} \quad\left(u \in \mathfrak{M}(E), u \mid v_{0}\right) \\
\gamma_{q} \neq 0 .
\end{array}\right.
$$

We prove that with this choice of $\gamma_{q}, \gamma_{1}, \ldots \gamma_{r_{t-1}}$ the vector $\boldsymbol{h}=\boldsymbol{h}_{q}$ in (9.37), (9.38) satisfies assertion (9.20) for $j=q$ and for $j=1, \ldots, n$.

We begin with $u \in \mathfrak{M}(E), u \mid v_{0}$.

For $i$ with $1 \leq i \leq r_{t-1}$ we use (9.40) and (9.46) to obtain

$$
\left\|h_{q i}\right\|_{u}=\left\|h_{i}\right\|_{u} \leq \max _{1 \leq j \leq r_{t-1}}\left\{\left\|\gamma_{q} \vartheta_{j}+\gamma_{j}\right\|_{u}\left\|h_{j i}\right\|_{u}\right\} \leq \max _{1 \leq j \leq r_{t-1}}\left\{\left\|h_{j i}\right\|_{u}\right\} .
$$

Now by the induction hypothesis

$$
\left\|h_{j i}\right\|_{u} \leq\left((1+\varepsilon)^{n+1} 2^{n^{2}} \min \left\{\lambda_{i}, \lambda_{j}\right\}\right)^{d\left(u / v_{0}\right)} \leq\left((1+\varepsilon)^{n+1} 2^{n^{2}} \lambda_{i}\right)^{d\left(u / v_{0}\right)}
$$

for $j=1, \ldots, r_{t-1}$ and for $i=1, \ldots, n$.

So indeed, since $r_{t-1}+1 \leq q \leq r_{t}$ we get

$$
\left\|h_{q i}\right\|_{u} \leq\left((1+\varepsilon)^{n+1} 2^{n^{2}} \min \left\{\lambda_{i}, \lambda_{q}\right\}\right)^{d\left(u / v_{0}\right)} \quad \text { for } i=1, \ldots, r_{t-1} .
$$

We next treat for $u \mid v_{0}$ the range $r_{t-1}+1 \leq i \leq n$. In view of (9.45), we may infer that for such $i$

$$
\left\|h_{q i}\right\|_{u}=\left\|h_{i}\right\|_{u} \leq \max \left\{\left\|\gamma_{q}\right\|_{u}\left\|g_{q i}\right\|_{u} ;\left\|a_{i k}\right\|_{u}\left\|h_{k}-\gamma_{q} g_{q k}\right\|_{u}\left(k=1, \ldots, r_{t-1}\right)\right\} .
$$

By (9.18), (9.46) and (9.14) and since $r_{t-1}+1 \leq q \leq r_{t}$

$$
\begin{aligned}
\left\|\gamma_{q}\right\|_{u}\left\|g_{q i}\right\|_{u} & \leq\left((1+\varepsilon)^{r_{t-1}+2} 2^{\left(r_{t-1}+1\right)^{2}} \lambda_{q}\right)^{d\left(u / v_{0}\right)} \\
& =\left((1+\varepsilon)^{r_{t-1}+2} 2^{\left(r_{t-1}+1\right)^{2}} \lambda_{r_{t-1}+1}\right)^{d\left(u / v_{0}\right)} \\
& \leq\left((1+\varepsilon)^{n+1} 2^{n^{2}} \lambda_{r_{t-1}+1}\right)^{d\left(u / v_{0}\right)}
\end{aligned}
$$

Moreover, by (9.41), (9.47) (which is already established), (9.46) and (9.18)

$$
\begin{aligned}
& \left\|a_{i k}\right\|_{u}\left\|h_{k}-\gamma_{q} g_{q k}\right\|_{u}=\left\|a_{i k}\right\|_{u}\left\|h_{q k}-\gamma_{q} g_{q k}\right\|_{u} \leq \\
\leq & \max \left\{\left\|h_{q k}\right\|_{u},\left\|\gamma_{q}\right\|_{u}\left\|g_{q k}\right\|_{u}\right\} \\
\leq & \max \left\{(1+\varepsilon)^{n+1} 2^{n^{2}} \lambda_{r_{t-1}},(1+\varepsilon)^{r_{t-1}+2} 2^{\left(r_{t-1}+1\right)^{2}} \lambda_{q}\right\}^{d\left(u / v_{0}\right)} \\
\leq & \left((1+\varepsilon)^{n+1} 2^{n^{2}} \lambda_{r_{t-1}+1}\right)^{d\left(u / v_{0}\right)} \quad \text { for } 1 \leq k \leq r_{t-1} .
\end{aligned}
$$


So altogether, for $r_{t-1}+1 \leq i \leq n$ we obtain

$$
\begin{aligned}
\left\|h_{q i}\right\|_{u} & \leq\left((1+\varepsilon)^{n+1} 2^{n^{2}} \lambda_{r_{t-1}+1}\right)^{d\left(u / v_{0}\right)} \\
& =\left((1+\varepsilon)^{n+1} 2^{n^{2}} \min \left\{\lambda_{i}, \lambda_{q}\right\}\right)^{d\left(u / v_{0}\right)}
\end{aligned}
$$

(the last equation since $r_{t-1}+1 \leq q \leq r_{t}$ and by (9.14)).

Inequalities (9.47) and (9.48) imply that assertion (9.20) is satisfied for $j=q, i=1, \ldots, n$ and for $u \in \mathfrak{M}(E)$ with $u \mid v_{0}$.

We still have to verify (9.20) for $u \nmid v_{0}$. We use (9.36) to get

$$
\left\|L_{i}^{(u)}\left(\boldsymbol{h}_{q}\right)\right\|_{u} \leq\left(1+r_{t-1}\right)^{s(u)} \max \left\{\left\|\gamma_{q}\right\|_{u}\left\|L_{i}^{(u)}\left(\boldsymbol{g}_{q}\right)\right\|_{u},\left\|\gamma_{j}\right\|_{u}\left\|L_{i}^{(u)}\left(\boldsymbol{h}_{j}\right)\right\|_{u}\left(1 \leq j \leq r_{t-1}\right)\right\} .
$$

By (9.46), (9.17) and the induction hypothesis this is

$$
\leq\left(1+r_{t-1}\right)^{s(u)}\left(1+r_{t-1}\right)^{-s(u)} Q^{c_{i u}}=Q^{c_{i u}}
$$

So (9.20) is satisfied for $u \in \mathfrak{M}(E), u \nmid v_{0}$, for $j=q$ and for $i=1, \ldots, n$.

Since $q$ with $r_{t-1}+1 \leq q \leq r_{t}$ is arbitrary it follows in fact from our construction and the induction hypothesis that (9.19) now is satisfied for $j=1, \ldots, r_{t}$ and (9.20) for $j=1, \ldots, r_{t}$ and for $i=1, \ldots, n$. The Lemma follows.

\section{Multilinear Algebra}

For $k$ with $1 \leq k \leq n$, we write $C(n, k)$ for the set of ordered $k$-tuples

$$
\sigma=\left\{1 \leq i_{1}<\ldots<i_{k} \leq n\right\}
$$

Put

$$
N=\left(\begin{array}{l}
n \\
k
\end{array}\right)
$$

and let $\tau_{1}, \ldots, \tau_{N}$ be the enumeration of $C(n, k)$ in the lexicographical ordering. For a field $F$ and vectors $\boldsymbol{a}_{1}, \ldots, \boldsymbol{a}_{k} \in F^{n}$ with $\boldsymbol{a}_{i}=\left(a_{i 1}, \ldots, a_{i n}\right)$ we define $\boldsymbol{a}_{1} \wedge \ldots \wedge \boldsymbol{a}_{k}=$ $\left(A_{1}, \ldots, A_{N}\right)$ by

$$
A_{i}=\operatorname{det}\left(\begin{array}{ccc}
a_{1, i_{1}} & \ldots & a_{1, i_{k}} \\
\vdots & & \vdots \\
a_{k, i_{1}} & \ldots & a_{k, i_{k}}
\end{array}\right) \quad(i=1, \ldots, N)
$$

where $\left\{i_{1}<\ldots<i_{k}\right\}=\tau_{i}$. Given linear forms $L_{i}=a_{i 1} X_{1}+\ldots+a_{i n} X_{n}$ $(i=1, \ldots, k)$, write $\boldsymbol{a}_{i}=\left(a_{i 1}, \ldots, a_{i n}\right)$ for the coefficient vectors and put $L_{1} \wedge \ldots \wedge L_{k}=$ $A_{1} X_{1}+\ldots+A_{N} X_{N}$, where $\left(A_{1}, \ldots, A_{N}\right)=\boldsymbol{a}_{1} \wedge \ldots \wedge \boldsymbol{a}_{k}$. 
Now let $L_{i}^{(v)}$ and $\boldsymbol{c}=\left(c_{i v}\right)(v \in \mathfrak{M}(K), i=1, \ldots, n)$ be as in (6.1) - (6.8). We define for $\sigma=\left\{i_{1}<\ldots<i_{k}\right\} \in C(n, k)$

$$
L_{\sigma}^{(v)}=L_{i_{1}}^{(v)} \wedge \ldots \wedge L_{i_{k}}^{(v)} \quad(v \in \mathfrak{M}(K))
$$

and

$$
c_{\sigma v}=c_{i_{1} v}+\ldots+c_{i_{k} v} \quad(v \in \mathfrak{M}(K)) .
$$

Notice that by (6.4)

$$
\operatorname{det}\left(L_{\tau_{1}}^{(v)}, \ldots, L_{\tau_{N}}^{(v)}\right)=\operatorname{det}\left(L_{1}^{(v)}, \ldots, L_{n}^{(v)}\right)^{\frac{k}{n} N}=1 .
$$

For a finite extension $E$ of $K$ and for $u \in \mathfrak{M}(E)$ lying above $v \in \mathfrak{M}(K)$ we define in analogy with $(6.8)$ for $\sigma \in C(n, k)$

$$
L_{\sigma}^{(u)}=L_{\sigma}^{(v)}, \quad c_{\sigma u}=d(u / v) c_{\sigma v} .
$$

Let $\mu_{1}, \ldots, \mu_{n}$ be positive real numbers. For $\sigma=\left\{i_{1}<\ldots<i_{k}\right\} \in C(n, k)$ we put

$$
\mu_{\sigma}=\mu_{i_{1}} \ldots \mu_{i_{k}}
$$

Given linearly independent points $\boldsymbol{h}_{1}, \ldots, \boldsymbol{h}_{n} \in \overline{\mathbb{Q}}^{n}$ we write

$$
\boldsymbol{h}_{\sigma}=\boldsymbol{h}_{i_{1}} \wedge \ldots \wedge \boldsymbol{h}_{i_{k}} \quad(\sigma \in C(n, k)) .
$$

Assume that the points $\boldsymbol{h}_{1}, \ldots, \boldsymbol{h}_{n}$ have components in the finite extension $E$ of $K$. Given a permutation $\pi$ of $\{1, \ldots, n\}$ and $\sigma=\left\{i_{1}<\ldots<i_{k}\right\} \in C(n, k)$ we write $\pi \sigma$ for the element in $C(n, k)$ consisting of $\pi\left(i_{1}\right), \ldots, \pi\left(i_{k}\right)$ (reordered according to increasing size). Now suppose that we have

$$
\left\{\begin{array}{r}
\left\|L_{i}^{(u)}\left(\boldsymbol{h}_{j}\right)\right\|_{u} \leq Q^{c_{i u}}\left(u \in \mathfrak{M}(E), u \nmid v_{0}, 1 \leq i, j \leq n\right) \\
\left\|L_{\pi(i)}^{(u)}\left(\boldsymbol{h}_{j}\right)\right\|_{u}=\left\|h_{j \pi(i)}\right\|_{u} \leq \min \left\{\mu_{i}, \mu_{j}\right\}^{d\left(u / v_{0}\right)} \\
\left(u \in \mathfrak{M}(E), u \mid v_{0}, 1 \leq i, j \leq n\right) .
\end{array}\right.
$$

We will also study the situation when (10.8) is replaced by the simpler hypothesis

$$
\left\{\begin{aligned}
&\left\|L_{i}^{(u)}\left(\boldsymbol{h}_{j}\right)\right\| \leq Q^{c_{i u}} \quad\left(u \in \mathfrak{M}(E), u \nmid v_{0}, 1 \leq i, j \leq n\right) \\
&\left\|L_{i}^{(u)}\left(\boldsymbol{h}_{j}\right)\right\|_{u}=\left\|h_{j i}\right\|_{u} \leq \mu_{j}^{d\left(u / v_{0}\right)} \quad\left(u \in \mathfrak{M}(E), u \mid v_{0}, 1 \leq i, j \leq n\right) .
\end{aligned}\right.
$$

For $\sigma \in C(n, k)$ we write $\boldsymbol{h}_{\sigma}=\left(h_{\sigma, \tau_{1}}, \ldots, h_{\sigma, \tau_{N}}\right)$.

Lemma 10.1 Let $\boldsymbol{h}_{1}, \ldots, \boldsymbol{h}_{n}$ be linearly independent points in $\overline{\mathbb{Q}}^{n}$ as above. Let $\mu_{1}, \ldots, \mu_{n}$ be positive real numbers. Suppose we have (10.8). Then there exist a finite extension $F$ 
of $E$ and an element $\beta \in F^{*}$ with the following property:

The points $\beta \boldsymbol{h}_{\tau_{1}}, \ldots, \beta \boldsymbol{h}_{\tau_{N}}$ satisfy the simultaneous inequalities

$$
\left\{\begin{array}{r}
\left\|L_{\tau_{i}}^{(w)}\left(\beta \boldsymbol{h}_{\tau_{j}}\right)\right\|_{w} \leq Q^{c_{\tau_{i} w}} \quad\left(w \in \mathfrak{M}(F), w \nmid v_{0}, 1 \leq i, j \leq N\right) \\
\left\|L_{\pi \tau_{i}}^{(w)}\left(\beta \boldsymbol{h}_{\tau_{j}}\right)\right\|_{w}=\left\|\beta h_{\tau_{j}, \pi \tau_{i}}\right\|_{w} \leq \quad\left(k^{k} \min \left\{\mu_{\tau_{i}}, \mu_{\tau_{j}}\right\}\right) \\
\left(w \in \mathfrak{M}(F), w \mid v_{0}, 1 \leq i, j \leq N\right) .
\end{array}\right.
$$

If instead of (10.8) we have (10.8a), then there exist $F$ and $\beta$ as above such that instead of (10.9) we have

$$
\left\{\begin{aligned}
\left\|L_{\tau_{i}}^{(w)}\left(\beta \boldsymbol{h}_{\tau_{j}}\right)\right\|_{w} & \leq Q^{c_{\tau_{i} w}} \quad\left(w \in \mathfrak{M}(F), w \nmid v_{0}, 1 \leq i, j \leq N\right) \\
\left\|L_{\tau_{i}}^{(w)}\left(\beta \boldsymbol{h}_{\tau_{j}}\right)\right\|_{w}=\left\|\beta h_{\tau_{j}, \tau_{i}}\right\|_{w} \leq\left(k^{k} \mu_{\tau_{j}}\right)^{d\left(w / v_{0}\right)} & \left(w \in \mathfrak{M}(F), w \mid v_{0}, 1 \leq i, j \leq N\right) .
\end{aligned}\right.
$$

Proof. We first deal with (10.9). We apply Lemma 6.3 with $F$ replaced by $K$ and with

$$
A_{v}=\left\{\begin{array}{cl}
\left(k !^{1 / k}\right)^{-d(v)} & \text { for } \quad v \in \mathfrak{M}_{\infty}(K) \\
(1+\varepsilon) k !^{1 / k} & \text { for } v=v_{0} \\
1 & \text { for } v \in \mathfrak{M}_{0}(K) \backslash\left\{v_{0}\right\}
\end{array}\right.
$$

where $\varepsilon>0$ is a small parameter to be specified later. (10.10) implies

$$
\prod_{v \in \mathfrak{M}(K)} A_{v}=1+\varepsilon>1
$$

So the analogue of (6.26) is true. Consequently we can find a finite extension $F$ of $K$ and an element $\gamma \in F^{*}$ satisfying

$$
\|\gamma\|_{w} \leq\left\{\begin{array}{cl}
\left(k !^{1 / k}\right)^{-d(w / v) d(v)} & \text { for } \quad w \in \mathfrak{M}_{\infty}(F), w \mid v, v \in \mathfrak{M}(K) \\
(1+\varepsilon) k !^{1 / k} & \text { for } w \in \mathfrak{M}(F), w \mid v_{0} \\
1 & \text { for } w \in \mathfrak{M}_{0}(F), w \nmid v_{0} .
\end{array}\right.
$$

We may assume that $F$ contains the field $E$. Combination of (10.8) and (10.11) yields

$$
\left\{\begin{aligned}
\left\|L_{i}^{(w)}\left(\gamma \boldsymbol{h}_{j}\right)\right\|_{w} \leq\left(k !^{1 / k}\right)^{-d(w)} Q^{c_{i w}} & \left(w \in \mathfrak{M}_{\infty}(F), 1 \leq i, j \leq n\right) \\
\left\|L_{\pi(i)}^{(w)}\left(\gamma \boldsymbol{h}_{j}\right)\right\|_{w}=\left\|\gamma h_{j \pi(i)}\right\|_{w} \leq\left((1+\varepsilon) k !^{1 / k} \min \left\{\mu_{i}, \mu_{j}\right\}\right) & \left(w\left(w / v_{0}\right)\right. \\
\left\|L_{i}^{(w)}\left(\gamma \boldsymbol{h}_{j}\right)\right\|_{w} \leq Q^{c_{i w}} & \left(w \in \mathfrak{M}(F), w \mid v_{0}, 1 \leq i, j \leq n\right) \\
& \left.(w), w \nmid v_{0}, 1 \leq i, j \leq n\right) .
\end{aligned}\right.
$$

Now suppose that $\sigma_{i}=\left\{i_{1}<\ldots<i_{k}\right\}, \sigma_{j}=\left\{j_{1}<\ldots<j_{k}\right\}$. Writing $\beta=\gamma^{k}$, we obtain using Laplace's identity

$$
L_{\sigma_{i}}^{(w)}\left(\beta \boldsymbol{h}_{\sigma_{j}}\right)=\operatorname{det}\left(\begin{array}{ccc}
L_{i_{1}}^{(w)}\left(\gamma \boldsymbol{h}_{j_{1}}\right) & \ldots & L_{i_{1}}^{(w)}\left(\gamma \boldsymbol{h}_{j_{k}}\right) \\
\vdots & & \vdots \\
L_{i_{k}}^{(w)}\left(\gamma \boldsymbol{h}_{j_{1}}\right) & \ldots & L_{i_{k}}^{(w)}\left(\gamma \boldsymbol{h}_{j_{k}}\right)
\end{array}\right)(w \in \mathfrak{M}(F))
$$


Combining (10.12) and (10.13) we may infer with (10.4) that

$$
\left\{\begin{aligned}
\left\|L_{\tau_{i}}^{(w)}\left(\beta \boldsymbol{h}_{\tau_{j}}\right)\right\|_{w} \leq k !^{s(w)} k !^{-s(w)} Q^{c_{\tau_{i} w}}= & Q^{c_{\tau_{i} w}} \\
& \left(w \in \mathfrak{M}(F), w \nmid v_{0}, 1 \leq i, j \leq n\right) \\
\left\|\beta h_{\tau_{j}, \pi \tau_{i}}\right\|_{w} \leq\left((1+\varepsilon)^{k} k ! \min \left\{\mu_{\tau_{i}}, \mu_{\tau_{j}}\right\}\right)^{d\left(w / v_{0}\right)} & \left(w \in \mathfrak{M}(F), w \mid v_{0}, 1 \leq i, j \leq n\right) .
\end{aligned}\right.
$$

Choosing $\varepsilon$ such that $(1+\varepsilon)^{k}<k$ we get with (10.14) assertion (10.9).

To prove $(10.9 \mathrm{a})$ we proceed in exactly the same way. Instead of (10.12) we get analogous inequalities with $\min \left(\mu_{i}, \mu_{j}\right)$ replaced by $\mu_{j}$ and with $\pi(i)$ replaced by $i$. Similarly, instead of (10.14) we obtain inequalities with $\min \left\{\mu_{\tau_{i}}, \mu_{\tau_{j}}\right\}$ replaced by $\mu_{\tau_{j}}$ and with $\pi \tau_{i}$ replaced by $\tau_{i}$. This completes the proof of Lemma 10.1 .

In section 12 we will be interested in the particular instance of the situation studied above when $k=n-1$. Then (10.1) becomes

$$
N=\left(\begin{array}{c}
n \\
n-1
\end{array}\right)=n
$$

The lexicographical ordering $\tau_{1}, \ldots, \tau_{n}$ of $C(n, n-1)$ is given by $\{1, \ldots, n-1\}, \ldots,\{2, \ldots, n\}$. So (10.3), (10.4) and (10.7) respectively become

$$
\begin{array}{ll}
L_{\tau_{n+1-i}}^{(v)}=L_{1}^{(v)} \wedge \ldots \wedge L_{i-1}^{(v)} \wedge L_{i+1}^{(v)} \wedge \ldots \wedge L_{n}^{(v)}=\widehat{L}_{i}^{(v)} & (i=1, \ldots, n), \\
c_{\tau_{n+1-i}, v}=c_{1 v}+\ldots+c_{i-1, v}+c_{i+1, v}+\ldots+c_{n v}=\widehat{c}_{i v} & (i=1, \ldots, n),
\end{array}
$$

and

$$
\boldsymbol{h}_{\tau_{n+1-i}}=\boldsymbol{h}_{1} \wedge \ldots \wedge \boldsymbol{h}_{i-1} \wedge \boldsymbol{h}_{i+1} \wedge \ldots \wedge \boldsymbol{h}_{n}=\widehat{\boldsymbol{h}}_{i} \quad(i=1, \ldots, n)
$$

say.

Lemma 10.2 Suppose $\mu_{1}, \ldots, \mu_{n}$ are positive real numbers. Let $E$ be a finite extension of $K$ and suppose $\boldsymbol{h}_{1}, \ldots, \boldsymbol{h}_{n}$ are linearly independent points in $E^{n}$ with (10.8a). Then there exist a finite extension $F$ of $E$ and an element $\beta \in F^{*}$ such that the point

$$
\beta \widehat{\boldsymbol{h}}_{n}=\beta \boldsymbol{h}_{1} \wedge \ldots \wedge \boldsymbol{h}_{n-1}
$$

satisfies

$$
\begin{aligned}
&\left\|\widehat{L}_{i}^{(w)}\left(\beta \widehat{\boldsymbol{h}}_{n}\right)\right\|_{w} \leq Q^{\widehat{c}_{i w}} \quad\left(w \in \mathfrak{M}(F), w \nmid v_{0}, 1 \leq i, j \leq n\right) \\
&\left\|\beta \widehat{h}_{n i}\right\|_{w} \leq\left((n-1)^{n-1} \mu_{1} \cdots \mu_{n-1}\right)^{d\left(w / v_{0}\right)} \\
&\left(w \in \mathfrak{M}(F), w \mid v_{0}, 1 \leq i, j \leq n\right)
\end{aligned}
$$

where $\widehat{\boldsymbol{h}}_{n}=\left(\widehat{h}_{n 1}, \ldots, \widehat{h}_{n n}\right)$. 
Proof. The assertion follows at once from (10.9a) with $k=n-1$ and $j=1$.

Lemma 10.3 Let $\boldsymbol{h}_{1}, \ldots, \boldsymbol{h}_{n}$ be linearly independent points $\overline{\mathbb{Q}}^{n}$. Suppose $1 \leq k \leq n$. Let $N$ be given by (10.1) and let $\sigma_{1}, \ldots, \sigma_{N}$ be an enumeration of $C(n, k)$ such that $\sigma_{N}=$ $\{n-k+1, n-k+2, \ldots, n\}$. Define the points $\boldsymbol{h}_{\sigma_{1}}, \ldots, \boldsymbol{h}_{\sigma_{N}}$ as in (10.6). Once the span of $\boldsymbol{h}_{\sigma_{1}}, \ldots, \boldsymbol{h}_{\sigma_{N-1}}$ in $\overline{\mathbb{Q}}^{N}$ is determined, the span of $\boldsymbol{h}_{1}, \ldots, \boldsymbol{h}_{n-k}$ is determined as well.

This is Lemma 6.4 of Schmidt [14].

\section{Heights}

For vectors $\boldsymbol{x} \in \overline{\mathbb{Q}}^{n}$ we will work with the height $H(\boldsymbol{x})$ introduced in (3.1) and (3.2). For a polynomial $P$ the height $H(P)$ will be the height of its vector of coefficients. The local heights $\|P\|_{v}$ are defined in analogy with (3.1).

As in section 10, for $1 \leq k \leq n$ we let $C(n, k)$ be the set of ordered $k$-tuples $\tau=$ $\left\{1 \leq i_{1}<\ldots<i_{k} \leq n\right\}$. We let $\tau_{1}, \ldots, \tau_{N}$ be the enumeration of $C(n, k)$ in the lexicographical ordering. Here $N=\left(\begin{array}{l}n \\ k\end{array}\right)$. For $k=n-1$, writing $\boldsymbol{a}_{1} \wedge \ldots \wedge \boldsymbol{a}_{n-1}=\left(A_{1}, \ldots, A_{n}\right)$ we define the vector $\left(\boldsymbol{a}_{1} \wedge \ldots \wedge \boldsymbol{a}_{n-1}\right)^{*}=\left(A_{n},-A_{n-1}, A_{n-2}, \ldots,(-1)^{n-1} A_{1}\right)$. For $\boldsymbol{a}, \boldsymbol{b} \in \overline{\mathbb{Q}}^{n}$ we let $\boldsymbol{a} \boldsymbol{b}=a_{1} b_{1}+\ldots+a_{n} b_{n}$ be the canonical bilinear form. For $\boldsymbol{b}, \boldsymbol{a}_{2}, \ldots, \boldsymbol{a}_{n} \in K^{n}$ we get

$$
\boldsymbol{b}\left(\boldsymbol{a}_{2} \wedge \ldots \wedge \boldsymbol{a}_{n}\right)^{*}=\operatorname{det}\left(\boldsymbol{b}, \boldsymbol{a}_{2}, \ldots, \boldsymbol{a}_{n}\right) .
$$

In particular this implies for any points $\boldsymbol{a}_{1}, \ldots, \boldsymbol{a}_{n-1} \in K^{n}$

$$
\boldsymbol{a}_{i}\left(\boldsymbol{a}_{1} \wedge \ldots \wedge \boldsymbol{a}_{n-1}\right)^{*}=0 \text { for } i=1, \ldots, n-1 .
$$

Moreover, for $\boldsymbol{a}_{1}, \ldots, \boldsymbol{a}_{k} \in K^{n}$ and for any $v \in \mathfrak{M}(K)$ we have

$$
\left\|\boldsymbol{a}_{1} \wedge \ldots \wedge \boldsymbol{a}_{k}\right\|_{v} \leq\left\|\boldsymbol{a}_{1}\right\|_{v} \ldots\left\|\boldsymbol{a}_{k}\right\|_{v} .
$$

For $k=n$ this gives

$$
\left\|\operatorname{det}\left(\boldsymbol{a}_{1} \wedge \ldots \wedge \boldsymbol{a}_{n}\right)\right\|_{v} \leq\left\|\boldsymbol{a}_{1}\right\|_{v} \ldots\left\|\boldsymbol{a}_{n}\right\|_{v} .
$$

Also by (11.3)

$$
H\left(\boldsymbol{a}_{1} \wedge \ldots \wedge \boldsymbol{a}_{k}\right) \leq H\left(\boldsymbol{a}_{1}\right) \ldots H\left(\boldsymbol{a}_{k}\right) .
$$

Lemma 11.1 Let $\boldsymbol{a}, \boldsymbol{b} \in K^{n}$ such that

$$
\boldsymbol{a b} \neq 0 .
$$

Then we have for any subset $T$ of $\mathfrak{M}(K)$

$$
\prod_{v \in T}\|\boldsymbol{a} \boldsymbol{b}\|_{v} \geq \prod_{v \notin T}\|\boldsymbol{a}\|_{v}^{-1}\|\boldsymbol{b}\|_{v}^{-1} .
$$


Proof. Using (11.6) and the product formula we obtain

$$
1=\prod_{v \in \mathfrak{M}(K)}\|\boldsymbol{a} \boldsymbol{b}\|_{v}=\prod_{v \in T}\|\boldsymbol{a} \boldsymbol{b}\|_{v} \prod_{v \notin T}\|\boldsymbol{a} \boldsymbol{b}\|_{v} .
$$

Combining this with

$$
\prod_{v \notin T}\|\boldsymbol{a} \boldsymbol{b}\|_{v} \leq \prod_{v \notin T}\|\boldsymbol{a}\|_{v} \prod_{v \notin T}\|\boldsymbol{b}\|_{v}
$$

we get the assertion.

Lemma 11.2 Let $A=\left(a_{i j}\right)(1 \leq i, j \leq n)$ be a matrix with entries in $K$ and with

$$
\operatorname{det} A=1 .
$$

Let $B$ be the inverse matrix. Write $\boldsymbol{a}_{i}=\left(a_{i 1}, \ldots, a_{i n}\right)$ for the row vectors in $A$ $(i=1, \ldots, n)$ and $\boldsymbol{b}_{j}=\left(b_{1 j}, \ldots, b_{n j}\right)(j=1, \ldots, n)$ for the column vectors in $B$. Then

$$
\boldsymbol{b}_{j}= \pm\left(\boldsymbol{a}_{1} \wedge \ldots \wedge \boldsymbol{a}_{j-1} \wedge \boldsymbol{a}_{j+1} \wedge \ldots \wedge \boldsymbol{a}_{n}\right)^{*} .
$$

This is an immediate consequence of (11.1) and (11.2).

Lemma 11.3 Let $\left\{\boldsymbol{a}_{1}, \ldots, \boldsymbol{a}_{s}\right\}$ be a family of points in $K^{n}$. Let $H$ be a quantity with

$$
H\left(\boldsymbol{a}_{i}\right) \leq H \quad(i=1, \ldots, s) .
$$

Suppose that there exists a point $\boldsymbol{h} \in \overline{\mathbb{Q}}^{n}, \boldsymbol{h} \neq \mathbf{0}$ with

$$
\boldsymbol{a}_{i} \boldsymbol{h}=0 \quad(i=1, \ldots, s) .
$$

Then there exists a point $\boldsymbol{h}_{0} \in \overline{\mathbb{Q}}^{n}, \boldsymbol{h}_{0} \neq \mathbf{0}$ satisfying (11.11) and having moreover

$$
H\left(\boldsymbol{h}_{0}\right) \leq H^{n-1} .
$$

Proof. By (11.11) $\operatorname{rank}\left\{\boldsymbol{a}_{1}, \ldots, \boldsymbol{a}_{s}\right\}=t<n$. Suppose without loss of generality that in fact $\operatorname{rank}\left\{\boldsymbol{a}_{1}, \ldots, \boldsymbol{a}_{t}\right\}=t$. By adding suitable $n-1-t$ among the canonical unit vectors $\boldsymbol{e}_{1}, \ldots, \boldsymbol{e}_{n}$, for simplicity say $\boldsymbol{e}_{t+1}, \ldots, \boldsymbol{e}_{n-1}$, we will have

$$
\operatorname{rank}\left\{\boldsymbol{a}_{1}, \ldots, \boldsymbol{a}_{t}, \boldsymbol{e}_{t+1}, \ldots, \boldsymbol{e}_{n-1}\right\}=n-1 .
$$

From (11.2) we obtain

$$
\boldsymbol{a}_{i}\left(\boldsymbol{a}_{1} \wedge \ldots \wedge \boldsymbol{a}_{t} \wedge \boldsymbol{e}_{t-1} \wedge \ldots \wedge \boldsymbol{e}_{n-1}\right)^{*}=0 \quad \text { for } i=1, \ldots, t .
$$

By our choice of $\boldsymbol{a}_{1}, \ldots, \boldsymbol{a}_{t}$ for any $j(1 \leq j \leq s), \boldsymbol{a}_{j}$ is a linear combination of $\boldsymbol{a}_{1}, \ldots, \boldsymbol{a}_{t}$. We may conclude that

$$
\boldsymbol{a}_{j}\left(\boldsymbol{a}_{1} \wedge \ldots \wedge \boldsymbol{a}_{t} \wedge \boldsymbol{e}_{t-1} \wedge \ldots \wedge \boldsymbol{e}_{n-1}\right)^{*}=0 \text { for } j=1, \ldots, s
$$


We put $\boldsymbol{h}_{0}=\left(\boldsymbol{a}_{1} \wedge \ldots \wedge \boldsymbol{a}_{t} \wedge \boldsymbol{e}_{t-1} \wedge \ldots \wedge \boldsymbol{e}_{n-1}\right)^{*}$.

Then by (11.5) and (11.10) we get

$$
H\left(\boldsymbol{h}_{0}\right) \leq H\left(\boldsymbol{a}_{1}\right) \ldots H\left(\boldsymbol{a}_{t}\right) \leq H^{t} \leq H^{n-1} .
$$

The assertion follows.

Now let $\left\{L_{1}, \ldots, L_{r}\right\}$ be our family of linear forms from (6.1), (6.2). Recall from (2.13)

$$
\mathcal{H}=\mathcal{H}\left(L_{1}, \ldots, L_{r}\right)=\prod_{v \in \mathfrak{M}(K)} \max _{i_{1}, \ldots, i_{n}}\left\|\operatorname{det}\left(L_{i_{1}}, \ldots, L_{i_{n}}\right)\right\|_{v} .
$$

For $v \in \mathfrak{M}(K)$ we put

$$
\mathcal{H}_{v}=\max _{i_{1}, \ldots, i_{n}}\left\|\operatorname{det}\left(L_{i_{1}}, \ldots, L_{i_{n}}\right)\right\|_{v}
$$

and

$$
s(v)=\left\{\begin{array}{cll}
{\left[K_{v}: \mathbb{R}\right] /[K: \mathbb{Q}]} & \text { for } & v \in \mathfrak{M}_{\infty}(K) \\
0 & \text { for } & v \in \mathfrak{M}_{0}(K) .
\end{array}\right.
$$

Notice that

$$
\sum_{v \in \mathfrak{M}(K)} s(v)=\sum_{v \in \mathfrak{M}_{\infty}(K)} s(v)=1
$$

Lemma 11.4 Let $T$ be a subset of $\mathfrak{M}(K)$. Then we have for $i=1, \ldots, r$

$$
\prod_{v \in T}\left\|L_{i}\right\|_{v} \leq \prod_{v \in T}\left(n^{s(v) / 2} \mathcal{H}_{v}\right) \leq\left(\prod_{v \in T} n^{s(v) / 2}\right) \mathcal{H}
$$

in particular

$$
H\left(L_{i}\right) \leq n^{1 / 2} \mathcal{H}
$$

Proof. Since $\left\{X_{1}, \ldots, X_{n}\right\} \subset\left\{L_{1}, \ldots, L_{r}\right\}$ the quantity $\mathcal{H}_{v}$ in (11.13) satisfies $\mathcal{H}_{v} \geq 1$. Moreover, we may conclude that

$$
\prod_{v \in T} \mathcal{H}_{v} \leq \mathcal{H}
$$

This proves the second part of (11.15). Write $L_{i}(\boldsymbol{X})=a_{i 1} X_{1}+\ldots+a_{i n} X_{n}$. Fix $v \in \mathfrak{M}(K)$ and suppose without loss of generality that $\left\|a_{i 1}\right\|_{v}=\max \left\{\left\|a_{i 1}\right\|_{v}, \ldots,\left\|a_{i n}\right\|_{v}\right\}$. Then

$$
\left\|L_{i}\right\|_{v} \leq n^{s(v) / 2}\left\|a_{i 1}\right\|_{v} .
$$

On the other hand, we have

$$
\mathcal{H}_{v} \geq\left\|\operatorname{det}\left(L_{i}, X_{2}, \ldots, X_{n}\right)\right\|_{v}=\left\|a_{i 1}\right\|_{v}
$$


and therefore

$$
\left\|L_{i}\right\|_{v} \leq n^{s(v) / 2} \mathcal{H}_{v}
$$

This proves the first part of (11.15).

Since $\sum_{v \in \mathfrak{M}(K)} s(v)=1,(11.16)$ is a special instance of (11.15).

Lemma 11.5 Let $i_{1}, \ldots, i_{n-1}$ be in $\{1, \ldots, r\}$ such that $L_{i_{1}}, \ldots, L_{i_{n-1}}$ are linearly independent. Then we have for any subset $T$ of $\mathfrak{M}(K)$

$$
\prod_{v \in T}\left\|L_{i_{1}} \wedge \ldots \wedge L_{i_{n-1}}\right\|_{v} \leq \prod_{v \in T} n^{s(v) / 2} \mathcal{H}_{v}
$$

in particular

$$
H\left(L_{i_{1}} \wedge \ldots \wedge L_{i_{n-1}}\right) \leq n^{1 / 2} \mathcal{H}
$$

Proof. Notice that the coefficients of $L_{i_{1}} \wedge \ldots \wedge L_{i_{n-1}}$ apart from sign and ordering are $\operatorname{det}\left(X_{1}, L_{i_{1}}, \ldots, L_{i_{n-1}}\right), \ldots, \operatorname{det}\left(X_{n}, L_{i_{1}}, \ldots, L_{i_{n-1}}\right)$.

Thus for any $v \in \mathfrak{M}(K)$ we get

$$
\left\|L_{i_{1}} \wedge \ldots \wedge L_{i_{n-1}}\right\|_{v} \leq n^{s(v) / 2} \max _{1 \leq i \leq n}\left\|\operatorname{det}\left(X_{i}, L_{i_{1}}, \ldots, L_{i_{n}}\right)\right\|_{v} .
$$

However, by definition and since $\left\{X_{1}, \ldots, X_{n}\right\} \subset\left\{L_{1}, \ldots, L_{r}\right\}$ we see that

$$
\mathcal{H}_{v} \geq \max _{1 \leq i \leq n}\left\|\operatorname{det}\left(X_{i}, L_{i_{1}}, \ldots, L_{i_{n}}\right)\right\|_{v}
$$

The Lemma follows.

Lemma 11.6 Let $i_{1}, \ldots, i_{n} \in\{1, \ldots, r\}$ and suppose that $\operatorname{det}\left(L_{i_{1}}, \ldots, L_{i_{n}}\right)=1$. Let $A$ be the coefficient matrix of $L_{i_{1}}, \ldots, L_{i_{n}}$. Then the entries of the inverse matrix $B=\left(b_{i j}\right)$ satisfy

$$
\left\|b_{i j}\right\|_{v} \leq \mathcal{H}_{v} \quad(v \in \mathfrak{M}(K), 1 \leq i, j \leq n)
$$

Proof. The assertion follows from combination of Lemma 11.2 and formula (11.19) in the above proof.

Lemma 11.7 Let $i_{1}, \ldots, i_{n-1} \in\{1, \ldots, r\}$ and let $\boldsymbol{g} \in K^{n}$. Suppose that we have

$$
\left(L_{i_{1}} \wedge \ldots \wedge L_{i_{n-1}}\right)(\boldsymbol{g}) \neq 0
$$

Then we get for any subset $T$ of $\mathfrak{M}(K)$

$$
\prod_{v \in T}\left\|\left(L_{i_{1}} \wedge \ldots \wedge L_{i_{n-1}}\right)(\boldsymbol{g})\right\|_{v} \geq\left(\prod_{v \notin T} n^{-s(v) / 2} \mathcal{H}_{v}^{-1}\right) \prod_{v \notin T}\|g\|_{v}^{-1} .
$$


Proof. Lemma 11.1 says that

$$
\prod_{v \in T}\left\|\left(L_{i_{1}} \wedge \ldots \wedge L_{i_{n-1}}\right)(\boldsymbol{g})\right\|_{v} \geq \prod_{v \notin T}\left\|L_{i_{1}} \wedge \ldots \wedge L_{i_{n-1}}\right\|_{v}^{-1} \prod_{v \notin T}\|g\|_{v}^{-1} .
$$

On the other hand by Lemma 11.5

$$
\prod_{v \notin T}\left\|L_{i_{1}} \wedge \ldots \wedge L_{i_{n-1}}\right\|_{v}^{-1} \geq \prod_{v \notin T} n^{-s(v) / 2} \mathcal{H}_{v}^{-1}
$$

The Lemma follows.

Lemma 11.8 Suppose that each form $L_{i}$ of our family $\left\{L_{1}, \ldots, L_{r}\right\}$ has some coefficient equal to 1 . Then

$$
\mathcal{H}\left(L_{1}, \ldots, L_{r}\right) \leq H\left(L_{1}\right) \ldots H\left(L_{r}\right)
$$

Proof. For $v \in \mathfrak{M}(K)$ let $i_{1}(v), \ldots, i_{n}(v)$ be such that

$$
\left\|\operatorname{det}\left(L_{i_{1}(v)}, \ldots, L_{i_{n}(v)}\right)\right\|_{v}=\max _{i_{1}, \ldots, i_{n}}\left\|\operatorname{det}\left(L_{i_{1}}, \ldots, L_{i_{n}}\right)\right\|_{v}=\mathcal{H}_{v} .
$$

Then (11.4) implies

$$
\mathcal{H}_{v} \leq\left\|L_{i_{1}(v)}\right\|_{v} \ldots\left\|L_{i_{n}(v)}\right\|_{v}
$$

However, our assumption on the forms $L_{i}$ yields

$$
\left\|L_{i}\right\|_{v} \geq 1 \quad \text { for } i=1, \ldots, r \text {. }
$$

In conjunction with (11.24) we may conclude that

$$
\mathcal{H}_{v} \leq\left\|L_{1}\right\|_{v} \ldots\left\|L_{r}\right\|_{v}
$$

and therefore

$$
\mathcal{H}=\prod_{v \in \mathfrak{M}(K)} \mathcal{H}_{v} \leq \prod_{v \in \mathfrak{M}(K)}\left(\left\|L_{1}\right\|_{v} \ldots\left\|L_{r}\right\|_{v}\right)=H\left(L_{1}\right) \ldots H\left(L_{r}\right)
$$

In Lemma 11.3 we have given an upper bound for the height of a solution of a system of linear equations in terms of the heights of the coefficient vectors. During our proof we will however also need another much more precise result in that context.

The following Lemma is an immediate consequence of Theorem 9 of Bombieri and Vaaler [1]. 
Lemma 11.9 Let $K$ be a number field of degree $d$. Write $\mathcal{D}_{K}$ for the absolute value of its dicriminant. Suppose

$$
m<n
$$

and let $\boldsymbol{a}_{1}, \ldots, \boldsymbol{a}_{m}$ be nonzero points in $K^{n}$. Then there exists a nonzero point $\boldsymbol{x} \in$ $K^{n} \backslash\{\mathbf{0}\}$ with

$$
\boldsymbol{a}_{i} \boldsymbol{x}=0 \quad \text { for } i=1, \ldots, m
$$

and

$$
H(\boldsymbol{x}) \leq n^{1 / 2} \mathcal{D}_{K}^{1 / 2 d}\left(H\left(\boldsymbol{a}_{1}\right) \ldots H\left(\boldsymbol{a}_{m}\right)\right)^{1 /(n-m)} .
$$

Remark. Since $H(\lambda \boldsymbol{x})=H(\boldsymbol{x})$ for any $\lambda \in \overline{\mathbb{Q}}^{*}$, we may clearly suppose that the vector $\boldsymbol{x}$ in Lemma 11.9 has integral components.

\section{The Height of the Penultimate Minimum Sub- space}

We consider the parallelepiped $\Pi(Q, \boldsymbol{c})$ defined in (6.10), (6.11) with forms $L_{i}^{(v)}$ and tuples $\boldsymbol{c}=\left(c_{i v}\right)(v \in \mathfrak{M}(K), i=1, \ldots, n)$ as in (6.1) - (6.8).

Again we let $\lambda_{1}=\lambda_{1}(Q), \ldots, \lambda_{n}=\lambda_{n}(Q)$ be the successive minima of $\Pi(Q, \boldsymbol{c})$ (according to $(6.12)-(6.14))$.

Suppose

$$
0<\delta<1
$$

We will study the set of $Q$ such that

$$
Q^{\delta}>n^{1 / 2}
$$

and

$$
\lambda_{n-1}=\lambda_{n-1}(Q) \leq Q^{-\delta}
$$

Corollary 7.6 says that $\lambda_{n}(Q) \geq n^{-1 / 2}$. Thus by (12.2), (12.3) there exists $\varepsilon>0$ with

$$
\lambda_{n-1}(1+\varepsilon)<\lambda_{n}
$$

Let $\boldsymbol{g}_{1}=\boldsymbol{g}_{1}(Q), \ldots, \boldsymbol{g}_{n}=\boldsymbol{g}_{n}(Q)$ be linearly independent points in $\overline{\mathbb{Q}}^{n}$ with

$$
\boldsymbol{g}_{i} \in(1+\varepsilon) \lambda_{i} * \Pi(Q, \boldsymbol{c}) \quad(1 \leq i \leq n) .
$$


Write $T=T(Q)$ for the linear subspace of $\overline{\mathbb{Q}}^{n}$ generated by $\boldsymbol{g}_{1}, \ldots, \boldsymbol{g}_{n-1}$. By Corollary 7.5 , given $Q$, the space $T$ is uniquely determined. In particular it does not depend upon $\varepsilon$, nor upon the choice of $\boldsymbol{g}_{1}, \ldots, \boldsymbol{g}_{n-1}$ with (12.5). However, in general $T$ will clearly depend on $Q$.

Given $T$, there is a vector $\boldsymbol{v} \neq \mathbf{0}$ in $\overline{\mathbb{Q}}^{n}$ such that $T$ consists of the points $\boldsymbol{x} \in \overline{\mathbb{Q}}^{n}$ with

$$
\boldsymbol{v} \boldsymbol{x}=0 .
$$

Up to a factor of proportionality $\boldsymbol{v}$ is uniquely determined. If $\boldsymbol{v}=\left(v_{1}, \ldots, v_{n}\right)$ we write $V(X)$ for the linear form $v_{1} X_{1}+\ldots+v_{n} X_{n}$.

In this section we will prove an assertion of the following type:

E $i$ t h e $r$ for each $Q$ under consideration which is sufficiently large the height $H(V)$ will be above some fixed positive power of $Q$

o $r T$ does not depend on $Q$ at all, i.e., there is a single subspace $T_{0}$, such that for all $Q$ under consideration we have

$$
T(Q)=T_{0} .
$$

For $\boldsymbol{g}=\left(g_{1}, \ldots, g_{n}\right) \in \overline{\mathbb{Q}}^{n}$ we put

$$
\boldsymbol{g}^{*}=\left(g_{n},-g_{n-1}, \ldots,(-1)^{n-1} g_{1}\right) .
$$

Moreover, in analogy with (10.18) we write

$$
\widehat{\boldsymbol{g}}_{n}=\boldsymbol{g}_{1} \wedge \cdots \wedge \boldsymbol{g}_{n-1}
$$

Since $\widehat{\boldsymbol{g}}_{n}^{*} \boldsymbol{x}=\operatorname{det}\left(\boldsymbol{g}_{1}, \ldots, \boldsymbol{g}_{n-1}, \boldsymbol{x}\right)$, the space $T$ consists of the points $\boldsymbol{x} \in \overline{\mathbb{Q}}^{n}$ satisfying

$$
\widehat{\boldsymbol{g}}_{n}^{*} \boldsymbol{x}=0 .
$$

So we may take $\boldsymbol{v}=\widehat{\boldsymbol{g}}_{n}^{*}$ and consequently

$$
H(V)=H\left(\widehat{\boldsymbol{g}}_{n}^{*}\right)=H\left(\widehat{\boldsymbol{g}}_{n}\right) .
$$

Let $\widehat{L}_{i}^{(v)}, \widehat{c}_{i v}(v \in \mathfrak{M}(K), i=1, \ldots, n)$ be defined as in (10.16), (10.17) respectively.

Lemma 12.1 Assume that we have (12.2) and (12.3). Let $R$ be the number of distinct sets $\left\{L_{1}^{(v)}, \ldots, L_{n}^{(v)}\right\}$ we are considering in $(6.1)-(6.7)$.

Moreover suppose that $v \mapsto i(v)(v \in \mathfrak{M}(K))$ is a map from $\mathfrak{M}(K)$ into $\{1, \ldots, n\}$ such that

$$
\widehat{L}_{i(v)}^{(v)}\left(\widehat{\boldsymbol{g}}_{n}\right) \neq 0 \quad \text { for each } v \in \mathfrak{M}(K) \text {. }
$$

Then

$$
H\left(\widehat{\boldsymbol{g}}_{n}\right)>n^{-1}(1+\varepsilon)^{-1} \mathcal{H}^{-1}\left(\left(\lambda_{1} \cdots \lambda_{n-1}\right)^{-1} \prod_{v \in \mathfrak{M}(K)} Q^{c_{i(v), v}}\right)^{\frac{1}{R n-1}}
$$


Proof. Let $E$ be a finite extension of $K$ such that $\boldsymbol{g}_{1}, \ldots, \boldsymbol{g}_{n} \in E^{n}$. We apply Lemma 10.2 with $\mu_{i}=(1+\varepsilon) \lambda_{i}$ and conclude that there exist a finite extension $F$ of $E$ and an element $\beta \in F^{*}$ with the following property:

For any tuple $(i(w))(w \in \mathfrak{M}(F))$ with $1 \leq i(w) \leq n$ the point $\beta \boldsymbol{g}_{n}=\beta \boldsymbol{g}_{1} \wedge \ldots \wedge \boldsymbol{g}_{n-1}$ satisfies

$$
\prod_{w \in \mathfrak{M}(F)}\left\|\widehat{L}_{i(w)}^{(w)}\left(\beta \boldsymbol{g}_{n}\right)\right\|_{w} \leq(1+\varepsilon)^{(n-1)}(n-1)^{n-1} \lambda_{1} \ldots \lambda_{n-1} \prod_{w \in \mathfrak{M}(F)} Q^{\widehat{c}_{i(w), w}}
$$

Now suppose that $(i(w))(w \in \mathfrak{M}(F))$ has the property that there exists $(i(v))(v \in \mathfrak{M}(K))$ such that

$$
i(w)=i(v) \quad \text { for } w \in \mathfrak{M}(F), w \mid v, v \in \mathfrak{M}(K) .
$$

For such a tuple we get

$$
\sum_{w \in \mathfrak{M}(F), w \mid v} c_{i(w), w}=c_{i(v), v}
$$

and thus in view of $(6.5),(10.17)$ we may infer from (12.12) that

$$
\prod_{w \in \mathfrak{M}(F)}\left\|\widehat{L}_{i(w)}^{(w)}\left(\beta \widehat{\boldsymbol{g}}_{n}\right)\right\|_{w} \leq(1+\varepsilon)^{(n-1)}(n-1)^{n-1} \lambda_{1} \ldots \lambda_{n-1} \prod_{v \in \mathfrak{M}(K)} Q^{-c_{i(v), v}}
$$

We now assume that the tuple $(i(v))(v \in \mathfrak{M}(K))$ satisfies (12.10) and we choose $(i(w))$ $(w \in \mathfrak{M}(F))$ as in (12.13). We will derive a lower bound for the left hand side of (12.14).

We partition $\mathfrak{M}(F)$ into $R$ subsets $T_{\rho}(\rho=1, \ldots, R)$. For $w \in T_{\rho}$, the system $\left\{L_{1}^{(w)}, \ldots, L_{n}^{(w)}\right\} \subset\left\{L_{1}, \ldots, L_{r}\right\}$ will be always the same, $\left\{L_{1}^{(\rho)}, \ldots, L_{n}^{(\rho)}\right\}$ say. We partition moreover $T_{\rho}$ into $n$ subsets $T_{\rho i}(i=1, \ldots, n)$. $T_{\rho i}$ consists of those $w \in T_{\rho}$ for which we have $i(w)=i$.

We apply Lemma 11.7 to obtain

$$
\prod_{w \in T_{\rho i}}\left\|\widehat{L}_{i}^{(w)}\left(\beta \widehat{\boldsymbol{g}}_{n}\right)\right\|_{w} \geq\left(\prod_{w \in T_{\rho i}}\|\beta\|_{w}\right) \prod_{w \notin T_{\rho i}}\left(n^{-\frac{1}{2} s(w)} \mathcal{H}_{w}^{-1}\left\|\widehat{\boldsymbol{g}}_{n}\right\|_{w}^{-1}\right) .
$$

Taking the product over $i$ and $\rho$ we get

$$
\prod_{w \in \mathfrak{M}(F)}\left\|\widehat{L}_{i(w)}^{(w)}\left(\beta \widehat{\boldsymbol{g}}_{n}\right)\right\|_{w} \geq\left(\prod_{w \in \mathfrak{M}(F)}\|\beta\|_{w}\right)\left(n^{-\frac{1}{2}} \mathcal{H}^{-1} H\left(\widehat{\boldsymbol{g}}_{n}\right)^{-1}\right)^{R n-1},
$$

and using the product formula we may conclude that

$$
\prod_{w \in \mathfrak{M}(F)}\left\|\widehat{L}_{i(w)}^{(w)}\left(\beta \widehat{\boldsymbol{g}}_{n}\right)\right\|_{w} \geq\left(n^{-\frac{1}{2}} \mathcal{H}^{-1} H\left(\widehat{\boldsymbol{g}}_{n}\right)^{-1}\right)^{R n-1} .
$$

Combination of (12.14) and (12.15) yields with $R>1$ (which we may assume)

$$
H\left(\widehat{\boldsymbol{g}}_{n}\right)^{R n-1} \geq n^{-(R n-1)}(1+\varepsilon)^{-(R n-1)}\left(\lambda_{1} \ldots \lambda_{n-1}\right)^{-1} \mathcal{H}^{-(R n-1)} \prod_{v \in \mathfrak{M}(K)} Q^{c_{i(v), v}}
$$


Taking $(R n-1)$-st roots we get assertion (12.11).

Let $\mathfrak{S}$ be the set of tuples $(j(v))(v \in \mathfrak{M}(K))(1 \leq j(v) \leq n)$ such that

$$
\sum_{v \in \mathfrak{M}(K)} c_{j(v), v} \geq 0
$$

Corollary 12.2 Suppose $\delta>0$ and

$$
\lambda_{n-1}=\lambda_{n-1}(Q) \leq Q^{-\delta}
$$

(i.e., (12.3)). Suppose moreover that

$$
Q^{\delta}>(n \mathcal{H})^{6 R} .
$$

Assume that there exists a tuple $(j(v)(v \in \mathfrak{M}(K))$ in the set $\mathfrak{S}$ defined by (12.16) with

$$
\widehat{L}_{j(v)}^{(v)}\left(\widehat{\boldsymbol{g}}_{n}\right) \neq 0 \quad \text { for each } v \in \mathfrak{M}(K) .
$$

Then

$$
H\left(\widehat{\boldsymbol{g}}_{n}\right)>Q^{\delta / 3 R}
$$

Proof. Let $(j(v))(v \in \mathfrak{M}(K))$ be a tuple in $\mathfrak{S}$ satisfying (12.19). Then Lemma 12.1 in conjunction with (12.17) implies

$$
H\left(\widehat{\boldsymbol{g}}_{n}\right)>n^{-1}(1+\varepsilon)^{-1} \mathcal{H}^{-1} Q^{\frac{n-1}{R n-1} \delta} .
$$

As we have seen at the beginning of this section, (12.17), (12.18) entail that the space generated by $\boldsymbol{g}_{1}(Q), \ldots, \boldsymbol{g}_{n-1}(Q)$ does not depend upon $\varepsilon$ with (12.4). In particular this implies that $H\left(\widehat{\boldsymbol{g}}_{n}\right)$ does not depend on $\varepsilon$. Therefore by (12.21)

$$
\begin{aligned}
H\left(\widehat{\boldsymbol{g}}_{n}\right) & \geq n^{-1} \mathcal{H}^{-1} Q^{\frac{n-1}{R n-1} \delta} \\
& >Q^{\frac{\delta}{2 R}} n^{-1} \mathcal{H}^{-1} .
\end{aligned}
$$

Assertion (12.20) now follows from hypothesis (12.18).

We now deal with the case when hypothesis (12.19) is not true. In other words, we consider the situation when for every tuple $(j(v))(v \in \mathfrak{M}(K))$ in the set $\mathfrak{S}$ from $(12.16)$ there exists $v \in \mathfrak{M}(K)$ such that

$$
\widehat{L}_{j(v)}^{(v)}\left(\widehat{\boldsymbol{g}}_{n}\right)=0 .
$$

Notice that $\widehat{\boldsymbol{g}}_{n}=\widehat{\boldsymbol{g}}_{n}(Q)$. Therefore condition (12.22) depends on $Q$. However (12.22) implies that there exists a vector $\boldsymbol{h} \in \overline{\mathbb{Q}}^{n}, \boldsymbol{h} \neq \mathbf{0}$ such that for each tuple $(j(v))$ $(v \in \mathfrak{M}(K))$ in the set $\mathfrak{S}$ there is $v \in \mathfrak{M}(K)$ with

$$
\widehat{L}_{j(v)}^{(v)}(\boldsymbol{h})=0 .
$$

Obviously (12.23) is independent of $Q$. 
Lemma 12.3 Suppose that there exists $\boldsymbol{h} \in \overline{\mathbb{Q}}^{n}, \boldsymbol{h} \neq 0$ satisfying (12.23).

Then there exists $\boldsymbol{h}_{0} \neq \mathbf{0}$ in $\overline{\mathbb{Q}}^{n}$ with the following property:

For every $Q$ with

$$
\lambda_{n-1}=\lambda_{n-1}(Q) \leq Q^{-\delta}
$$

and

$$
Q^{-\delta}>n^{n} \mathcal{H}^{n}
$$

we have

$$
\boldsymbol{g}_{1}(Q) \boldsymbol{h}_{0}^{*}=\ldots=\boldsymbol{g}_{n-1}(Q) \boldsymbol{h}_{0}^{*}=0,
$$

where $\boldsymbol{h}_{0}^{*}$ is defined in analogy with (12.6).

Proof. Using hypothesis (12.23) we may apply Lemmata 11.3 and 11.4 to conclude that there exists indeed a point $\boldsymbol{h}_{0} \neq \mathbf{0}$ in $\overline{\mathbb{Q}}^{n}$ satisfying (12.23) and

$$
H\left(\boldsymbol{h}_{0}\right) \leq n^{\frac{1}{2}(n-1)} \mathcal{H}^{n-1} .
$$

Fix such a point $\boldsymbol{h}_{0}$ and write

$$
\boldsymbol{h}_{0}=\left(h_{1}, \ldots, h_{n}\right) \text {. }
$$

Let $\boldsymbol{q}_{1}, \ldots, \boldsymbol{q}_{n-1}$ be linearly independent points in $\overline{\mathbb{Q}}^{n}$ satisfying

$$
\boldsymbol{h}_{0}^{*} \boldsymbol{q}_{i}=0 \quad(i=1, \ldots, n-1) .
$$

Then $\boldsymbol{q}_{1} \wedge \ldots \wedge \boldsymbol{q}_{n-1}$ is a nonzero multiple of $\boldsymbol{h}_{0}$, and in fact $\boldsymbol{q}_{1}, \ldots, \boldsymbol{q}_{n-1}$ may be chosen such that

$$
\boldsymbol{q}_{1} \wedge \ldots \wedge \boldsymbol{q}_{n-1}=\boldsymbol{h}_{0}
$$

To prove (12.26), in view of (12.28) it suffices to prove that

$$
\operatorname{det}\left(\boldsymbol{q}_{1}, \ldots, \boldsymbol{q}_{n-1}, \boldsymbol{g}_{i}(Q)\right)=0 \quad(i=1, \ldots, n-1) .
$$

Let $F$ be a finite extension of $K$ such that $\boldsymbol{q}_{1}, \ldots, \boldsymbol{q}_{n-1}, \boldsymbol{g}_{1}, \ldots, \boldsymbol{g}_{n-1} \in F^{n}$. To verify (12.30) ist suffices to prove

$$
\prod_{w \in \mathfrak{M}(F)}\left\|\operatorname{det}\left(\boldsymbol{q}_{1}, \ldots, \boldsymbol{q}_{n-1}, \boldsymbol{g}_{i}\right)\right\|_{w}<1 \quad \text { for } i=1, \ldots, n-1 .
$$

Using (6.4) and Laplace's identy we get for $w \in \mathfrak{M}(F)$

$$
\left\{\begin{array}{c}
\left\|\operatorname{det}\left(\boldsymbol{q}_{1}, \ldots, \boldsymbol{q}_{n-1}, \boldsymbol{g}_{i}\right)\right\|_{w}=\left\|\begin{array}{cccc}
L_{1}^{(w)}\left(\boldsymbol{q}_{1}\right) & \ldots & L_{1}^{(w)}\left(\boldsymbol{q}_{n-1}\right) & L_{1}^{(w)}\left(\boldsymbol{g}_{i}\right) \\
\vdots & & \vdots & \vdots \\
L_{n}^{(w)}\left(\boldsymbol{q}_{1}\right) & \ldots & L_{n}^{(w)}\left(\boldsymbol{q}_{n-1}\right) & L_{n}^{(w)}\left(\boldsymbol{g}_{i}\right)
\end{array}\right\|_{w} \leq \\
\leq n^{s(w)} \max _{1 \leq j \leq n}\left\|L_{j}^{(w)}\left(\boldsymbol{g}_{i}\right) \cdot \widehat{L}_{j}^{(w)}\left(\boldsymbol{q}_{1} \wedge \ldots \wedge \boldsymbol{q}_{n-1}\right)\right\|_{w} \leq \\
\leq n^{s(w)} \max _{1 \leq j \leq n}\left\|L_{j}^{(w)}\left(\boldsymbol{g}_{i}\right)\right\|_{w} \cdot\left\|\widehat{L}_{j}^{(w)}\left(\boldsymbol{h}_{0}\right)\right\|_{w} .
\end{array}\right.
$$


For $v \in \mathfrak{M}(K)$ we define

$$
\mathfrak{I}_{v}=\left\{i \in\{1, \ldots, n\} \mid \widehat{L}_{i}^{(v)}\left(\boldsymbol{h}_{0}\right) \neq 0\right\} .
$$

Pick $k(v) \in \mathfrak{I}_{v}$ such that

$$
c_{i v} \leq c_{k(v), v} \quad \text { for each } i \in \mathfrak{I}_{v} .
$$

Our definition of the set $\mathfrak{S}$ in (12.16) together with (12.23), (12.33), (12.34) implies that

$$
\sum_{\mathfrak{M}(K)} c_{k(v), v}<0 .
$$

For each place $w \in \mathfrak{M}(K)$ with $w \mid v$ we put $\mathfrak{I}_{w}=\mathfrak{I}_{v}$ and $k(w)=k(v)$. Then (12.32) and the definition of $\mathfrak{I}_{w}$ entail

$$
\left\|\operatorname{det}\left(\boldsymbol{q}_{1}, \ldots, \boldsymbol{q}_{n-1}, \boldsymbol{g}_{i}\right)\right\|_{w} \leq n^{s(w)} \max _{j \in \mathfrak{I}_{w}}\left\|L_{j}^{(w)}\left(\boldsymbol{g}_{i}\right)\right\|_{w}\left\|\widehat{L}_{j}^{(w)}\left(\boldsymbol{h}_{0}\right)\right\|_{w} .
$$

Now by (12.5)

$\left\|L_{j}^{(w)}\left(\boldsymbol{g}_{i}\right)\right\|_{w} \leq Q^{c_{j w}} \quad\left(w \in \mathfrak{M}(F), w \nmid v_{0}, 1 \leq j \leq n, 1 \leq i \leq n-1\right)$

$\left\|L_{j}^{(w)}\left(\boldsymbol{g}_{i}\right)\right\|_{w} \leq Q^{c_{j w}}\left((1+\varepsilon) \lambda_{n-1}\right)^{d\left(w / v_{0}\right)} \quad\left(w \in \mathfrak{M}(F), w \mid v_{0}, 1 \leq j \leq n, 1 \leq i \leq n-1\right)$.

On the other hand by Lemma 11.5 we have

$$
\left\|\widehat{L}_{j}^{(w)}\left(\boldsymbol{h}_{0}\right)\right\|_{w} \leq n^{s(w) / 2} \mathcal{H}_{w}\left\|\boldsymbol{h}_{0}\right\|_{w} .
$$

Combining (12.36) with these two estimates and observing also (12.24) we obtain

$$
\left\|\operatorname{det}\left(\boldsymbol{q}_{1}, \ldots, \boldsymbol{q}_{n-1}, \boldsymbol{g}_{i}\right)\right\|_{w} \leq\left\{\begin{array}{r}
n^{3 s(w) / 2} \mathcal{H}_{w}\left\|\boldsymbol{h}_{0}\right\|_{w} \max _{j \in \mathfrak{I}_{w}} Q^{c_{j w}} \quad\left(w \in \mathfrak{M}(F), w \nmid v_{0}\right) \\
n^{3 s(w) / 2} \mathcal{H}_{w}\left\|\boldsymbol{h}_{0}\right\|_{w}(1+\varepsilon)^{d\left(w / v_{0}\right)} \max _{j \in \mathfrak{I}_{w}} Q^{c_{j w}-\delta d\left(w / v_{0}\right)} \\
\left(w \in \mathfrak{M}(F), w \mid v_{0}\right) .
\end{array}\right.
$$

Taking the product over $w \in \mathfrak{M}(F)$ and using (12.27), (12.34), (12.35) we may infer that

$$
\begin{aligned}
\prod_{w \in \mathfrak{M}(F)}\left\|\operatorname{det}\left(\boldsymbol{q}_{1}, \ldots, \boldsymbol{q}_{n-1}, \boldsymbol{g}_{i}\right)\right\|_{w} & \leq n^{\frac{1}{2}(n+1)} \mathcal{H}^{n}(1+\varepsilon) Q^{-\delta+\sum_{w \in \mathfrak{M}(F)} c_{k(w), w}} \\
& <n^{\frac{1}{2}(n+1)} \mathcal{H}^{n}(1+\varepsilon) Q^{-\delta}
\end{aligned}
$$

Finally, taking $\varepsilon$ sufficiently small, we get with (12.25)

$$
\prod_{w \in \mathfrak{M}(F)}\left\|\operatorname{det}\left(\boldsymbol{q}_{1}, \ldots, \boldsymbol{q}_{n-1}, \boldsymbol{g}_{i}\right)\right\|_{w}<n^{n} \mathcal{H}^{n} Q^{-\delta}<1
$$

and this is the desired (12.31).

Combining Corollary 12.2 and Lemma 12.3 we may summarize the results proved in this section as follows: 
Lemma 12.4 Suppose $\delta>0$. Consider the set of $Q$ with

$$
\lambda_{n-1}=\lambda_{n-1}(Q) \leq Q^{-\delta}
$$

and

$$
Q^{\delta}>(n \mathcal{H})^{\max \{n, 6 R\}}
$$

Then one of the following two alternatives is true:

(i) For all $Q$ under consideration the linear form $V=V(Q)$ has height

$$
H(V)=H\left(\widehat{\boldsymbol{g}}_{n}\right)>Q^{\delta / 3 R} .
$$

(ii) There exists a single proper subspace $T_{0}$ of $\overline{\mathbb{Q}}^{n}$ such that for all $Q$ under consideration we have $T(Q)=T_{0}$.

Proof. Notice that in view of (12.37) and (12.38) we have (12.24), (12.25) as well as (12.17), (12.18).

Now, if there exists $Q$ such that $\widehat{\boldsymbol{g}}_{n}(Q)$ does not satisfy (12.19), then clearly hypothesis (12.23) is satisfied and we get with Lemma 12.3 alternative (ii).

Otherwise for all $Q$ hypothesis (12.19) is true and Corollary 12.2 gives alternative (i).

\section{The Index}

We write $\mathfrak{R}$ for the ring of polynomials

$$
P=P\left(X_{11}, \ldots, X_{1 n} ; \ldots ; X_{m 1}, \ldots, X_{m n}\right)
$$

in $m n$ variables and with coefficients in $\overline{\mathbb{Q}}$. Let

$$
\boldsymbol{r}=\left(r_{1}, \ldots, r_{m}\right)
$$

be an $m$-tuple of natural numbers and

$$
\mathfrak{I}=\left(i_{11}, \ldots, i_{1 n} ; \ldots ; i_{m 1}, \ldots, i_{m n}\right)
$$

an $m n$-tuple of nonnegative integers. Put

$$
\mathfrak{I} / \boldsymbol{r}=\sum_{h=1}^{m} \frac{i_{h 1}+\ldots+i_{h n}}{r_{h}}
$$

and

$$
P^{\mathfrak{I}}=\frac{1}{i_{11} ! \ldots i_{m n} !} \frac{\partial^{i_{11}+\ldots+i_{m n}}}{\partial X_{11}^{i_{11}} \ldots \partial X_{m n}^{i_{m n}}} P
$$


Now suppose $P$ is a polynomial in $\Re$ and let $K$ be a number field containing its coefficients. We write $H(P)$ for the height of the coefficient vector of $P$, and for $v \in \mathfrak{M}(K)$ we denote the local height by $\|P\|_{v}$. If $P$ has degree $r_{h}$ in the block of variables $X_{h 1}, \ldots, X_{h n}(h=$ $1, \ldots, m)$ then, putting

$$
q=r_{1}+\ldots+r_{m}
$$

we have for any $\mathfrak{I}$ and for $v \in \mathfrak{M}(K)$

$$
\left\|P^{\mathfrak{I}}\right\|_{v} \leq 2^{q s(v)}\|P\|_{v}
$$

and consequently

$$
H\left(P^{\mathfrak{I}}\right) \leq 2^{q} H(P) .
$$

(For a proof cf. Schmidt [13], p. 172, Lemma 5A).

Let $D_{1}, \ldots, D_{m}$ be nonzero linear forms with coefficients in $\overline{\mathbb{Q}}$, such that for $h=1, \ldots, m$, $D_{h}$ is a form in the variables $X_{h 1}, \ldots, X_{h n}$. Let $T$ be the linear subspace of $\overline{\mathbb{Q}}^{m n}$ defined by the equations

$$
D_{1}=\ldots=D_{m}=0 .
$$

Following Schmidt [13] (p. $166 \mathrm{ff}$.) we define the index of a polynomial $P \in \mathfrak{R}$ with respect to $\left(D_{1}, \ldots, D_{m} ; \boldsymbol{r}\right)$ as follows:

When $P \equiv 0$, we set ind $(P)=\infty$. When $P \not \equiv 0$, the index ind $(P)$ is the least value of $c$ such that there exists $\mathfrak{I}$ with $\mathfrak{I} / \boldsymbol{r}=c$ and such that $P^{\mathfrak{I}}$ does not vanish identically on $T$.

The following fact is proved in [13] (Lemma 4C, p. 171). If our forms $D_{h}$ are of the shape

$$
D_{h}=\alpha_{h 1} X_{h 1}+\ldots+\alpha_{h n} X_{h n}
$$

with $\alpha_{h 1} \neq 0$ for $h=1, \ldots, m$, then there exists

$$
\mathfrak{I}=\left(i_{1}, 0, \ldots, 0 ; \ldots ; i_{m}, 0, \ldots, 0\right)
$$

with $\mathfrak{I} / \boldsymbol{r}=$ ind $(P)$ and with

$$
P^{\mathfrak{I}} \not \equiv 0 \quad \text { on } T \text {. }
$$

We now quote Evertse's version of Roth's Lemma ([4], Lemma 24). He derived it from his explicit version [3] of Faltings' Product Theorem [6].

We write $\mathfrak{R}(\boldsymbol{r})$ for the set of polynomials in $\mathfrak{R}$ which are homogeneous of degree $r_{h}$ in the block of variables $X_{h 1}, \ldots, X_{h n}(h=1, \ldots, m)$.

Proposition 13.1 Let $m, n$ be integers $\geq 2$. Let $0<\vartheta \leq 1$ and suppose $\boldsymbol{r}=\left(r_{1}, \ldots, r_{m}\right)$ satisfies

$$
r_{h} / r_{h+1} \geq \frac{2 m^{2}}{\vartheta} \quad(h=1, \ldots, m-1)
$$


Let $P$ be a nonzero polynomial in $\mathfrak{R}(\boldsymbol{r})$. Suppose that for $h=1, \ldots, m$ we are given linear forms $V_{h}$ with coefficients in $\overline{\mathbb{Q}}$ in the variables $X_{h 1}, \ldots, X_{h n}$. Assume that

$$
H\left(V_{h}\right)^{r_{h}} \geq\left\{\exp \left(r_{1}+\ldots+r_{m}\right) H(P)\right\}^{(n-1)\left(\frac{3 m^{2}}{\vartheta}\right)^{m}} \quad(h=1, \ldots, m) .
$$

Then the index of $P$ with respect to $\left(V_{1}, \ldots, V_{m} ; \boldsymbol{r}\right)$ satisfies

$$
\text { ind } P<m \vartheta \text {. }
$$

\section{The Approximation Polynomial}

The set $\mathfrak{R}(\boldsymbol{r})$ defined in the last section consists of polynomials

$$
P=\sum c\left(j_{11}, \ldots, j_{m n}\right) X_{11}^{j_{11}} \ldots X_{m}^{j_{m n}}
$$

where the summation is over $j_{i k} \geq 0$ with

$$
j_{h 1}+\ldots+j_{h n}=r_{h} \quad \text { for } h=1, \ldots, m .
$$

For each $h$, the number of such $j_{h 1}, \ldots, j_{h n}$ equals

$$
\left(\begin{array}{c}
r_{h}+n-1 \\
n-1
\end{array}\right)
$$

Hence the number of summands in (14.1) is

$$
M=\left(\begin{array}{c}
r_{1}+n-1 \\
n-1
\end{array}\right) \ldots\left(\begin{array}{c}
r_{m}+n-1 \\
n-1
\end{array}\right) \leq 2^{r_{1}+n-1+\ldots+r_{m}+n-1}=2^{q+m(n-1)},
$$

where we have put

$$
q=r_{1}+\ldots+r_{m}
$$

Lemma 14.1 Suppose $D=\alpha_{1} X_{1}+\ldots+\alpha_{n} X_{n}$ has coefficients $\alpha_{i}$ in a number field $K$ and $\alpha_{1} \neq 0$. Let $P$ be given by (14.1). Construct a polynomial $P^{*}$ in the $m n-m$ variables

$$
X_{12}, \ldots, X_{1 n} ; \ldots ; X_{m 2}, \ldots, X_{m n}
$$

by setting

$$
\begin{aligned}
P^{*}=P^{\mathfrak{I}}\left(-\alpha_{2} X_{12}-\ldots\right. & -\alpha_{n} X_{1 n}, \alpha_{1} X_{12}, \ldots, \alpha_{1} X_{1 n} ; \ldots ; \\
& \left.-\alpha_{2} X_{m 2}-\ldots-\alpha_{n} X_{m n}, \alpha_{1} X_{m 2}, \ldots, \alpha_{1} X_{m n}\right),
\end{aligned}
$$

with $\mathfrak{I}$ as in (13.4), i.e.,

$$
\mathfrak{I}=\left(i_{1}, 0, \ldots, 0 ; \ldots ; i_{m}, 0, \ldots, 0\right) .
$$

Then every coefficient $\gamma$ of $P^{*}$ is a linear form $\gamma=L_{\gamma}\left(\left(c\left(j_{11}, \ldots, j_{m n}\right)\right)\right)$ in the $M$ coefficients $c\left(j_{11}, \ldots, j_{m n}\right)$ of $P$. The coefficients of $L_{\gamma}$ lie in $K$ and each $L_{\gamma} \neq 0$ has

$$
H\left(L_{\gamma}\right)<2^{m n}\left(3 n^{1 / 2} H(D)\right)^{q} .
$$


This is Lemma 9.1 of Schmidt [14].

Now, given a linear form $D=\alpha_{1} X_{1}+\ldots+\alpha_{n} X_{n}$, we make $m$ forms out of it by setting $D_{[h]}=\alpha_{1} X_{h 1}+\ldots+\alpha_{n} X_{h n}(h=1, \ldots, m)$. The index with respect to $(D, \boldsymbol{r})$ is defined as the index with respect to $\left(D_{[1]}, \ldots, D_{[m]} ; \boldsymbol{r}\right)$.

Lemma 14.2 (Index Theorem) Suppose that $D_{1}, \ldots, D_{s}$ are nonzero linear forms with coefficients in a number field $K$ of degree $d$. Let $\mathcal{D}_{K}$ be the absolute value of the discriminant of $K$. Let $H$ be a quantitiy such that

$$
H\left(D_{t}\right) \leq H \quad \text { for } t=1, \ldots, s .
$$

Let $\vartheta>0$ and suppose that

$$
m>4 \vartheta^{-2} \log (2 s)
$$

Then given $\boldsymbol{r}=\left(r_{1}, \ldots, r_{m}\right)$, there exists a nonzero polynomial $P \in \mathfrak{R}(\boldsymbol{r})$ with integral algebraic coefficients in $K$ satisfying

$$
\operatorname{ind} P \geq\left(\frac{1}{n}-\vartheta\right) m
$$

with respect to $\left(D_{t}, \boldsymbol{r}\right)$ for $t=1, \ldots, s$;

$$
H(P)<\mathcal{D}_{K}^{\frac{1}{2 d}} 2^{\frac{3}{2} m n}\left(6 n^{\frac{1}{2}} H\right)^{q} .
$$

This is essentially the Index Theorem as in Schmidt [14], section 9. Since we give here a version where $P$ has coefficients in $K$ and not necessarily in $\mathbb{Q}$, we detail nevertheless the necessary changes as compared with [14].

Proof. We write $P$ as in (14.1). The number $M$ of available coefficients is given by (14.2). To deal with condition (i) for a particular form $D_{t}=D=\alpha_{1} X_{1}+\ldots+\alpha_{n} X_{n}$, say, we proceed as follows.

Without loss of generality, we suppose $\alpha_{1} \neq 0$. Then in view of (13.4), (13.5) the index condition will be satisfied for $D$ if for every $\mathfrak{I}$ of type (14.4) (i.e., (13.4)) with

$$
(\mathfrak{I} / \boldsymbol{r})<\left(\frac{1}{n}-\vartheta\right) m
$$

$P^{\mathfrak{I}}$ vanishes on the subspace $T$ defined by the equations $D_{[1]}=\ldots=D_{[m]}=0$. This means that for each such $\mathfrak{I}$ the corresponding polynomial $P^{*}$ as defined in Lemma 1.1 vanishes identically.

$P^{*}$ is homogeneous in $X_{h 2}, \ldots, X_{h n}$ of degree $r_{h}-i_{h}(h=1, \ldots, m)$. Thus $P^{*}$ has

$$
\left(\begin{array}{c}
r_{1}-i_{1}+n-2 \\
n-2
\end{array}\right) \ldots\left(\begin{array}{c}
r_{m}-i_{m}+n-2 \\
n-2
\end{array}\right)
$$


potential coefficients $\gamma$. In order to assure that $P^{*} \equiv 0$, each of the coefficients $\gamma$ has to be set equal to zero.

However, by Lemma 14.1 each $\gamma$ is a linear form $L_{\gamma}$ in the coefficients $c\left(j_{11}, \ldots, j_{m n}\right)$ of $P$. So for a single form $D$ and a single $\mathfrak{I}$, we get (14.8) linear equations in the $c\left(j_{11}, \ldots, j_{m N}\right)$. Summing over $t$ from 1 to $s$ (for the forms $D_{1}, \ldots, D_{s}$ ) and over $\mathfrak{I}$ we infer from (14.8) that altogether the $c\left(j_{11}, \ldots, j_{m N}\right)$ have to satisfy

$$
U=s \sum\left(\begin{array}{c}
r_{1}-i_{1}+n-2 \\
n-2
\end{array}\right) \ldots\left(\begin{array}{c}
r_{m}-i_{m}+n-2 \\
n-2
\end{array}\right)
$$

linear equations, where the sum is over nonnegative integers $i_{1}, \ldots, i_{m}$ with

$$
\frac{i_{1}}{r_{1}}+\ldots+\frac{i_{m}}{r_{m}}<\left(\frac{1}{n}-\vartheta\right) m
$$

By [13] (Lemma 4C, formula (4.3), p. 124) we obtain the estimate

$$
U<s\left(\begin{array}{c}
r_{1}+n-1 \\
n-1
\end{array}\right) \ldots\left(\begin{array}{c}
r_{m}+n-1 \\
n-1
\end{array}\right) e^{-\vartheta^{2} m / 4}=s M e^{-\vartheta^{2} m / 4} .
$$

So, using hypothesis (14.7) we see that

$$
U<\frac{1}{2} M
$$

Write $\boldsymbol{a}_{\gamma}$ for the coefficient vector of the linear form $L_{\gamma}$ and $\boldsymbol{c}$ for the vector with components $c\left(j_{11}, \ldots, j_{m n}\right)$. Then $\boldsymbol{a}_{\gamma} \in K^{M}$ and $\boldsymbol{c}$ should be a nonzero solution of the system of equations

$$
\boldsymbol{a}_{\gamma} \boldsymbol{x}=0 \text { for all } \gamma \text {. }
$$

We apply Lemma 11.9 and conclude that the system (14.9) has a nonzero solution $\boldsymbol{c} \in K^{M}$ with

$$
H(\boldsymbol{c}) \leq M^{1 / 2} \mathcal{D}_{K}^{\frac{1}{2 d}}\left(\max _{\gamma} H\left(\boldsymbol{a}_{\gamma}\right)\right)^{\frac{U}{M-U}} .
$$

Indeed we may suppose that $\boldsymbol{c}$ has integral components. Hence we obtain a nonzero polynomial $P$ with integral coefficients in $K$ satisfying

$$
\begin{aligned}
H(P) & \leq M^{1 / 2} \mathcal{D}_{K}^{\frac{1}{2 d}}\left(\max _{\gamma} H\left(\boldsymbol{a}_{\gamma}\right)\right)^{\frac{U}{M-U}} \\
& =M^{1 / 2} \mathcal{D}_{K}^{\frac{1}{2 d}}\left(\max _{\gamma} H\left(L_{\gamma}\right)\right)^{\frac{U}{M-U}} \\
& \leq M^{1 / 2} \mathcal{D}_{K}^{\frac{1}{2 d}} \max _{\gamma} H\left(L_{\gamma}\right) .
\end{aligned}
$$

Combining this with (14.2), (14.5) we get in fact

$$
H(P) \leq \mathcal{D}_{K}^{\frac{1}{2 d}} 2^{\frac{3}{2} m n}\left(6 n^{1 / 2} \max _{1 \leq t \leq s} H\left(D_{t}\right)\right)^{q} .
$$


By (14.6) this gives the assertion in (ii).

We will now adjust the general setting of our construction to the special situation we encounter when dealing with the linear forms in (6.1) - (6.8).

From now on we suppose that the polynomial in the Index Theorem has been constructed with respect to the forms $\left\{L_{1}, \ldots, L_{r}\right\}$ we start with. Then the parameter $s$ in the Index Theorem becomes

$$
s=r .
$$

We assume moreover that the number of distinct systems $\left\{L_{1}^{(v)}, \ldots, L_{n}^{(v)}\right\} \subset\left\{L_{1}, \ldots, L_{r}\right\}$ under consideration in (6.3) equals $R$. Then we get a partition $\mathfrak{M}(K)=\mathfrak{M}_{1} \cup \ldots \cup \mathfrak{M}_{R}$ such that for $v \in \mathfrak{M}_{\rho}$ the system $\left\{L_{1}^{(v)}, \ldots, L_{n}^{(v)}\right\}$ is always the same, $\left\{L_{1}^{(\rho)}, \ldots, L_{n}^{(\rho)}\right\}$ $(\rho=1, \ldots, R)$, say.

Given an $m n$-tuple $\mathfrak{I}$ and given $v \in \mathfrak{M}(K)$ we may write $P^{\mathfrak{I}}$ uniquely as

$$
P^{\mathfrak{I}}=\sum_{j_{11}, \ldots, j_{m n}} d_{v}^{\mathfrak{I}}\left(j_{11}, \ldots, j_{m n}\right) L_{1[1]}^{(v) j_{11}} \ldots L_{n[1]}^{(v) j_{1 n}} \ldots L_{m[1]}^{(v) j_{m 1}} \ldots L_{n[m]}^{(v) j_{m n}}
$$

where the sum is restricted to $j_{h 1}+\ldots+j_{h n} \leq r_{h}(h=1, \ldots, m)$.

Lemma 14.3 (Polynomial Theorem) Let $P$ be the polynomial of the Index Theorem, constructed with respect to $L_{1}, \ldots, L_{r}$. Suppose we have $(6.1)-(6.8)$. Let $\mathcal{H}$ be the quantitiy defined in (2.13).

Then with the representation of $P^{\mathfrak{I}}$ in (14.11) we obtain

(i) For each $v \in \mathfrak{M}(K)$, when $\mathfrak{I} / \boldsymbol{r} \leq 2 \vartheta$ m then $d_{v}^{\mathfrak{I}}\left(j_{11}, \ldots, j_{m n}\right)=0$ unless

$$
\left|\left(\sum_{h=1}^{m} \frac{j_{h k}}{r_{h}}\right)-\frac{m}{n}\right| \leq 3 m n \vartheta \quad \text { for } k=1, \ldots, n
$$

(ii)

$$
\prod_{v \in \mathfrak{M}(K)} \max _{j_{11}, \ldots, j_{m n}}\left\|d_{v}^{\mathfrak{I}}\left(j_{11}, \ldots, j_{m n}\right)\right\|_{v} \leq \mathcal{D}_{K}^{\frac{1}{2 d}}\left(2^{5 n+5} \mathcal{H}^{2}\right)^{q}
$$

Proof. (i) is exactly as in Schmidt [13] (p. 182 f.). As for (ii) we use the partition $\mathfrak{M}(K)=\mathfrak{M}_{1} \cup \ldots \cup \mathfrak{M}_{R}$. We fix $\rho(1 \leq \rho \leq R)$ and we study $v \in \mathfrak{M}_{\rho}$. Recall that $\left\{L_{1}^{(v)}, \ldots, L_{n}^{(v)}\right\}=\left\{L_{1}^{(\rho)}, \ldots, L_{n}^{(\rho)}\right\}$. For $j=1, \ldots, m$ and $i=1, \ldots, n$ we write

$$
X_{j i}=\gamma_{i 1}^{(\rho)} L_{1[j]}^{(\rho)}+\ldots+\gamma_{i n}^{(\rho)} L_{n[j]}^{(\rho)} .
$$

Substituting in (14.11), we get for a typical monomial in $P^{\mathfrak{I}}$

$$
X_{m n}^{j_{11}} \ldots X_{m n}^{j_{m n}}=\left(\sum_{k=1}^{n} \gamma_{1 k}^{(\rho)} L_{k[1]}^{(\rho)}\right)^{j_{11}} \ldots\left(\sum_{k=1}^{n} \gamma_{n k}^{(\rho)} L_{k[m]}^{(\rho)}\right)^{j_{m n}} .
$$


This is a polynomial in $L_{1[1]}^{(\rho)}, \ldots, L_{n[m]}^{(\rho)}$. For $v \in \mathfrak{M}_{\rho}$ its coefficients have modulus

$$
\leq\left(n^{s(v)} \max _{1 \leq i, k \leq n}\left\|\gamma_{i k}\right\|_{v}\right)^{j_{11}+\ldots+j_{m n}}
$$

On the other hand, by (13.2)

$$
\left\|P^{\mathfrak{I}}\right\|_{v} \leq 2^{q s(v)}\|P\|_{v} .
$$

Notice that $P^{\mathfrak{I}}$ is homogeneous in each block of variables. Therefore the sum $j_{11}+\ldots+j_{m n}$ in the exponent in (14.13) does not depend upon the particular monomial under consideration. Consequently (14.13) is true for any monomial. More precisely we may write

$$
j_{11}+\ldots+j_{m n}=l, \text { with } l \leq q,
$$

say, (where $q$ is given in (14.3)). The number of monomials in $P^{\mathfrak{I}}$ is $\leq M<2^{q+m n}$ by (14.2).

Combining (14.13), (14.14), (14.15) we may infer that for each $v \in \mathfrak{M}_{\rho}$

$$
\max _{j_{11}, \ldots, j_{m n}}\left\|d_{v}^{\mathfrak{I}}\left(j_{11}, \ldots, j_{m n}\right)\right\|_{v} \leq\left(2^{2 q+m n} n^{q}\right)^{s(v)}\|P\|_{v}\left(\max _{1 \leq i, k \leq n}\left\|\gamma_{i k}^{(\rho)}\right\|_{v}\right)^{l}
$$

and so we get

$$
\begin{aligned}
& \prod_{v \in \mathfrak{M}_{\rho}} \max _{j_{11}, \ldots, j_{m n}}\left\|d_{v}^{\mathfrak{I}}\left(j_{11}, \ldots, j_{m n}\right)\right\|_{v} \leq \\
& \quad \leq \prod_{v \in \mathfrak{M}_{\rho}}\left(\left(2^{2 q+m n} n^{q}\right)^{s(v)}\|P\|_{v}\left(\max _{1 \leq i, k \leq n}\left\|\gamma_{i k}^{(\rho)}\right\|_{v}\right)^{l}\right)
\end{aligned}
$$

Lemma 11.6 says that the $\gamma_{i k}^{(\rho)}$ given by (14.12) satisfy the estimate

$$
\prod_{v \in \mathfrak{M}_{\rho}} \max _{1 \leq i, k \leq n}\left\|\gamma_{i k}^{(\rho)}\right\|_{v} \leq \prod_{v \in \mathfrak{M}_{\rho}} \mathcal{H}_{v}
$$

Thus (14.16) yields

$$
\prod_{v \in \mathfrak{M}_{\rho}} \max _{j_{11}, \ldots, j_{m n}}\left\|d_{v}^{\mathfrak{I}}\left(j_{11}, \ldots, j_{m n}\right)\right\|_{v} \leq \prod_{v \in \mathfrak{M}_{\rho}}\left(2^{2 q+m n} n^{q}\right)^{s(v)}\|P\|_{v} \mathcal{H}_{v}^{q}
$$

Taking the product over $\rho$ we obtain

$$
\prod_{v \in \mathfrak{M}(K)} \max _{j_{11}, \ldots, j_{m n}}\left\|d_{v}^{\mathfrak{I}}\left(j_{11}, \ldots, j_{m n}\right)\right\|_{v} \leq 2^{2 q+m n} n^{q} \mathcal{H}^{q} H(P) .
$$

Introducing the bound for $H(P)$ from Lemma 14.2 and observing, that we use that Lemma with $L_{1}, \ldots, L_{r}$ in place of $D_{1}, \ldots, D_{s}$, we get with Lemma 11.4

$$
\begin{aligned}
H(P) & \leq \mathcal{D}_{K}^{\frac{1}{2 d}} 2^{\frac{3}{2} m n}\left(6 n^{1 / 2} \max _{1 \leq i \leq r} H\left(L_{i}\right)\right)^{q} \\
& \leq \mathcal{D}_{K}^{\frac{1}{2 d}} 2^{\frac{3}{2} m n}(6 n \mathcal{H})^{q} .
\end{aligned}
$$


Together with (14.18) we may infer that

$$
\prod_{v \in \mathfrak{M}(K)} \max _{j_{11}, \ldots, j_{m n}}\left\|d_{v}^{\mathfrak{I}}\left(j_{11}, \ldots, j_{m n}\right)\right\|_{v} \leq \mathcal{D}_{K}^{\frac{1}{2 d}}\left(2^{5 n+5} \mathcal{H}^{2}\right)^{q}
$$

and this is the assertion in (ii).

\section{The Index with Respect to $V\left(Q_{1}\right), \ldots, V\left(Q_{m}\right)$}

Again we consider our forms $L^{(v)}, \ldots, L_{n}^{(v)}(v \in \mathfrak{M}(K))$ and tuples $\boldsymbol{c}=\left(c_{i v}\right)$ $(v \in \mathfrak{M}(K), i=1, \ldots, n)$ from $(6.1)-(6.8)$. $\Pi(Q, c)$ is the parallelepiped defined in (6.10), (6.11) and $\lambda * \Pi(Q, \boldsymbol{c})$ is as in (6.13) - (6.15).

Let $\lambda_{1}(Q), \ldots, \lambda_{n}(Q)$ be the successive minima of $\Pi(Q, \boldsymbol{c})$ and $\boldsymbol{g}_{1}=\boldsymbol{g}_{1}(Q), \ldots, \boldsymbol{g}_{n-1}=$ $\boldsymbol{g}_{n-1}(Q)$ are linearly independent points in $\overline{\mathbb{Q}}^{n}$ with

$$
\boldsymbol{g}_{i}(Q) \in(1+\varepsilon) \lambda_{i} * \Pi(Q, \boldsymbol{c})
$$

i.e., with (12.5). $\varepsilon$ is a small positive number. We construct the point $\widehat{\boldsymbol{g}}_{n}=$ $=\boldsymbol{g}_{1} \wedge \ldots \wedge \boldsymbol{g}_{n-1}=\widehat{\boldsymbol{g}}_{n}(Q)$ and we write $\boldsymbol{v}(Q)=\widehat{\boldsymbol{g}}_{n}^{*}(Q)$ in analogy with (12.6). $\boldsymbol{v}(Q)$ is orthogonal to $\boldsymbol{g}_{1}, \ldots, \boldsymbol{g}_{n-1}$ and, as we have seen in the discussion at the beginning of section $12, \boldsymbol{v}(Q)$ is unique up to a factor in $\overline{\mathbb{Q}}^{*}$, provided that we have

$$
Q^{\delta}>n^{1 / 2}, \quad \lambda_{n-1} \leq Q^{-\delta}
$$

and provided that $\varepsilon$ is chosen such that

$$
\lambda_{n-1}(1+\varepsilon)<\lambda_{n}
$$

We let $V=V(Q)$ be the linear form with coefficient vector $\boldsymbol{v}(Q)$. If $V(Q)=$ $v_{1} X_{1}+\ldots+v_{n} X_{n}$ we write for $h=1, \ldots, m \quad V_{[h]}(Q)=v_{1} X_{h 1}+\ldots+v_{n} X_{h n}$.

We suppose that $\vartheta>0$ and $m>4 \vartheta^{-2} \log (2 r)$ such that (14.7) with $s=r$ is satisfied. We suppose moreover that $P$ is the polynomial of the Index and Polynomial Theorems in section 14 .

Lemma 15.1 Suppose that $0<\delta<1$ and that

$$
0<\vartheta \leq \frac{\delta}{20 n^{2}}
$$

Let $Q_{1}, \ldots, Q_{m}$ satisfy

$$
r_{1} \log Q_{1} \leq r_{h} \log Q_{h} \leq(1+\vartheta) r_{1} \log Q_{1} \quad(h=1, \ldots, m)
$$




$$
\lambda_{n-1}\left(Q_{h}\right) \leq Q_{h}^{-\delta} \quad(h=1, \ldots, m)
$$

and

$$
Q_{h}^{\delta}>2^{25 n} \mathcal{H}^{5} \mathcal{D}_{K}^{5 / 4 d q} \vartheta^{-5 / 2} \quad(h=1, \ldots, m)
$$

Then $P$ has index $\geq m \vartheta$ with respect to $\left(V_{[1]}\left(Q_{1}\right), \ldots, V_{[m]}\left(Q_{m}\right) ; \boldsymbol{r}\right)$.

It will be convenient to prove first the following auxiliary result:

For $v \in \mathfrak{M}(K)$ put

$$
c_{v}=\max \left\{c_{1 v}, \ldots, c_{n v}\right\}
$$

and define the tuple $\boldsymbol{b}=\left(b_{i v}\right)(v \in \mathfrak{M}(K), i=1, \ldots, n)$ by

$$
b_{i v}=\left\{\begin{array}{cl}
c_{i v}-c_{v} & \text { for } \quad v \in \mathfrak{M}(K), v \neq v_{0}, i=1, \ldots, n \\
\sum_{v \in \mathfrak{M}(K)} c_{v} & \text { for } \quad v=v_{0}, i=1, \ldots, n
\end{array}\right.
$$

Then by (6.5), (6.7), (6.12) and (15.8) we get

$$
\begin{gathered}
\sum_{v \in \mathfrak{M}(K)} \sum_{i=1}^{n} b_{i v}=0 \\
\max \left\{b_{1 v}, \ldots, b_{n v}\right\}=0 \quad\left(v \in \mathfrak{M}(K), v \neq v_{0}\right) \\
0 \leq b_{1 v_{0}}=\ldots=b_{n v_{0}} \leq 1 .
\end{gathered}
$$

For a finite extension $F$ of $K$ we define for $w \in \mathfrak{M}(F)$ lying above $v \in \mathfrak{M}(K)$ and for $i=1, \ldots, n$

$$
b_{i w}=d(w / v) b_{i v} .
$$

Lemma 15.2 Let $Q_{1}, \ldots, Q_{m}$ be as in Lemma 15.1. Let $\boldsymbol{g}_{1}(Q), \ldots, \boldsymbol{g}_{n-1}\left(Q_{1}\right), \ldots$, $\boldsymbol{g}_{1}\left(Q_{m}\right), \ldots, \boldsymbol{g}_{n-1}\left(Q_{m}\right)$ be points satisfying the analogue of $(15.1)$. Then there exist a finite extension $F$ of $K$ and elements $\beta_{h} \in F^{*}(h=1, \ldots, m)$ with the following property: The points $\boldsymbol{g}_{j}^{\prime}\left(Q_{h}\right)=\beta_{h} \boldsymbol{g}_{j}\left(Q_{h}\right)(1 \leq j \leq n-1,1 \leq h \leq m)$ all lie in $F^{n}$ and satisfy the inequalities

$$
\begin{aligned}
& \left\|L_{i}^{(w)}\left(\boldsymbol{g}_{j}^{\prime}\left(Q_{h}\right)\right)\right\|_{w} \leq Q_{h}^{b_{i w}} \\
& \left(w \in \mathfrak{M}(F), w \nmid v_{0} ; i=1, \ldots, n ; j=1, \ldots, n-1 ; h=1, \ldots, m\right) \\
& \left\|L_{i}^{(w)}\left(\boldsymbol{g}_{j}^{\prime}\left(Q_{h}\right)\right)\right\|_{w} \leq Q_{h}^{b_{i w}}\left((1+\varepsilon)^{2} \lambda_{j}\right)^{d\left(w / v_{0}\right)} \\
& \quad\left(w \in \mathfrak{M}(F), w \mid v_{0} ; i=1, \ldots, n ; j=1, \ldots, n-1 ; h=1, \ldots, m\right) .
\end{aligned}
$$


Proof. We apply Lemma 6.3 with $F$ replaced by $K$. Fix $h$ with $1 \leq h \leq m$ and put for $v \in \mathfrak{M}(K)$

$$
A_{v}= \begin{cases}Q_{h}^{-c_{v}} & \text { for } v \neq v_{0} \\ (1+\varepsilon) Q_{h}^{v \in \mathfrak{M}(K)} c_{v} & \text { for } v=v_{0} .\end{cases}
$$

Since $c_{v_{0}}=0$ by $(6.7)$, we obtain

$$
\prod_{v \in \mathfrak{M}(K)} A_{v}=1+\varepsilon>1
$$

So by Lemma 6.3 we can find a finite extension $F_{h}$ of $K$ and an element $\beta_{h} \in F_{h}^{*}$ satisfying

$$
\left\|\beta_{h}\right\|_{w} \leq A_{v}^{d(w / v)} \quad \text { for each } w \in F_{h}, w \mid v, v \in \mathfrak{M}(K)
$$

We may perform this construction for $h=1, \ldots, m$. Choosing the field $F$ so large that $F_{h} \subset F(h=1, \ldots, m)$ and such that moreover $\boldsymbol{g}_{i}\left(Q_{h}\right) \in F^{n}$ for $i=1, \ldots, n-1$ and $h=1, \ldots, m$, the assertion follows easily in view of (15.1) and (6.13) - (6.15).

We now turn to the proof of Lemma 15.1. Let $T$ be the subspace of $\overline{\mathbb{Q}}^{m n}$ defined by

$$
V_{[1]}\left(Q_{1}\right)=\ldots=V_{[m]}\left(Q_{m}\right)=0 .
$$

It suffices to show that $P^{\mathfrak{I}}=0$ on $T$ whenever $\mathfrak{I} / \boldsymbol{r}<\vartheta m$.

The points $\boldsymbol{g}_{1}^{\prime}\left(Q_{h}\right), \ldots, \boldsymbol{g}_{n-1}^{\prime}\left(Q_{h}\right)$ constructed in Lemma 15.2 obviously are a basis of the subspace of $\overline{\mathbb{Q}}^{n}$ defined by $V\left(Q_{h}\right)=0(h=1, \ldots, m)$. For $h=1, \ldots, m$ let $\Gamma_{h}$ be the grid consisting of points

$$
\boldsymbol{u}=u_{1} \boldsymbol{g}_{1}^{\prime}\left(Q_{h}\right)+\ldots+u_{n-1} \boldsymbol{g}_{n-1}^{\prime}\left(Q_{h}\right)
$$

where $u_{1}, \ldots, u_{n-1}$ run through the integers in $1 \leq u_{i} \leq\left[\vartheta^{-1}\right]+1$. It is shown in Schmidt [13] (p. 189) that it suffices to prove that

$$
P^{\mathfrak{I}}\left(\boldsymbol{u}_{1}, \ldots, \boldsymbol{u}_{m}\right)=0
$$

when $\mathfrak{I} / \boldsymbol{r}<2 \vartheta m$ and $\boldsymbol{u}_{h} \in \Gamma_{h}(h=1, \ldots, m)$. Let $F$ be the field obtained in Lemma 15.2. To verify (15.15), by the product formula it will suffice to show that

$$
\prod_{w \in \mathfrak{M}(F)}\left\|P^{\mathfrak{I}}\left(\boldsymbol{u}_{1}, \ldots, \boldsymbol{u}_{m}\right)\right\|_{w}<1
$$

whenever $\mathfrak{I} / \boldsymbol{r}<2 \vartheta m$ and $\boldsymbol{u}_{h} \in \Gamma_{h}(h=1, \ldots, m)$. In analogy with (14.11), for $w \in$ $\mathfrak{M}(F)$ we write $P^{\mathfrak{I}}\left(\boldsymbol{u}_{1}, \ldots, \boldsymbol{u}_{m}\right)$ uniquely as

$$
P^{\mathfrak{I}}\left(\boldsymbol{u}_{1}, \ldots, \boldsymbol{u}_{m}\right)=\sum_{j_{11}, \ldots, j_{m n}} d_{w}^{\mathfrak{I}}\left(j_{11}, \ldots, j_{m n}\right) L_{1}^{(w)}\left(\boldsymbol{u}_{1}\right)^{j_{11}} \ldots L_{n}^{(w)}\left(\boldsymbol{u}_{m}\right)^{j_{m n}}
$$


To verify (15.16), we first study the terms $L_{1}^{(w)}\left(\boldsymbol{u}_{1}\right)^{j_{11}} \ldots L_{n}^{(w)}\left(\boldsymbol{u}_{m}\right)^{j_{m n}}$ in (15.17). It clearly suffices to deal with exponents $\left(j_{11}, \ldots, j_{m n}\right)$ such that $d_{w}^{\mathfrak{I}}\left(j_{11}, \ldots, j_{m n}\right) \neq 0$. Combining (15.14) and (15.13) we obtain

$$
\begin{array}{r}
\left\|L_{k}^{(w)}\left(\boldsymbol{u}_{h}\right)\right\|_{w} \leq n^{s(w)}\left(\vartheta^{-1}+1\right)^{s(w)} Q_{h}^{b_{k w}} \leq\left(\frac{2 n}{\vartheta}\right)^{s(w)} Q_{h}^{b_{k w}} \\
\left(w \in \mathfrak{M}(F), w \nmid v_{0}, 1 \leq k \leq n, 1 \leq h \leq m\right) \\
\left\|L_{k}^{(w)}\left(\boldsymbol{u}_{h}\right)\right\|_{w} \leq\left(\frac{2 n}{\vartheta}\right)^{s(w)} Q_{h}^{b_{k w}}\left((1+\varepsilon)^{2} Q_{h}^{-\delta}\right)^{d\left(w / v_{0}\right)} \\
\left(w \in \mathfrak{M}(F), w \mid v_{0}, 1 \leq k \leq n, 1 \leq h \leq m\right) .
\end{array}
$$

Therefore

$$
\left\{\begin{array}{r}
\left\|L_{k}^{(w)}\left(\boldsymbol{u}_{1}\right)^{j_{1 k}} \ldots L_{k}^{(w)}\left(\boldsymbol{u}_{m}\right)^{j_{m k}}\right\|_{w} \leq\left(\frac{2 n}{\vartheta}\right)^{s(w)\left(j_{1 k}+\ldots+j_{m k}\right)}\left(Q_{1}^{j_{1 k}} \ldots Q_{m}^{j_{m k}}\right)^{b_{k w}} \\
\left(w \nmid v_{0}, 1 \leq k \leq n\right) \\
\left\|L_{k}^{(w)}\left(\boldsymbol{u}_{1}\right)^{j_{1 k}} \ldots L_{k}^{(w)}\left(\boldsymbol{u}_{m}\right)^{j_{m k}}\right\|_{w} \leq\left(\left(\frac{2 n}{\vartheta}\right)^{s(w)}(1+\varepsilon)^{2 d\left(w / v_{0}\right)}\right)^{\left(j_{1 k}+\ldots+j_{m k}\right)} \\
\left(Q_{1}^{j_{1 k}} \ldots Q_{m}^{j_{m k}}\right)^{b_{k w}-\delta d\left(w / v_{0}\right)}\left(w \mid v_{0}, 1 \leq k \leq n\right) .
\end{array}\right.
$$

Combination of (15.5) with assertion (i) of the Polynomial Theorem yields

$$
d_{w}^{\mathfrak{I}}\left(j_{11}, \ldots, j_{m n}\right)=0
$$

unless

$$
\sum_{h=1}^{m} j_{h k} \log Q_{h} \geq r_{1} \log Q_{1} \sum_{h=1}^{m} \frac{j_{h k}}{r_{h}} \geq r_{1} \log Q_{1}\left(\frac{1}{n}-3 n \vartheta\right) m
$$

and

$$
\sum_{h=1}^{m} j_{h k} \log Q_{h} \leq(1+\vartheta) r_{1} \log Q_{1} \sum_{h=1}^{m} \frac{j_{h k}}{r_{h}} \leq r_{1} \log Q_{1}(1+\vartheta)\left(\frac{1}{n}+3 n \vartheta\right) m .
$$

Using (15.18) - (15.20), (15.11), (15.12) and the fact that our tuples $\left(j_{11}, \ldots, j_{m n}\right)$ satisfy $j_{11}+\ldots+j_{m n} \leq q$, we may infer that

$$
\left\{\begin{array}{l}
\prod_{\substack{w \in \mathfrak{M}(F) \\
w \nmid v_{0}}} \underset{\substack{\max _{11}, \ldots, j_{m n} \\
\max ^{*}}}{\leq} \prod_{\substack{w \in \mathfrak{M}(F) \\
w \nmid v_{0}}}\left(\left(\frac{2 n}{\vartheta}\right)^{s(w) q} Q_{1}^{r_{1}\left(\frac{m}{n}-3 m n \vartheta\right)\left(b_{1 w}+\ldots+b_{n w}\right)}\right)
\end{array}\right.
$$

where the $*$ means that the maximum is taken over all tuples $\left(j_{11}, \ldots, j_{m n}\right)$ such that $d_{w}^{\mathfrak{I}}\left(j_{11}, \ldots, j_{m n}\right) \neq 0$. For $w \mid v_{0}$ we use moreover the estimate

$$
(1+\vartheta)\left(\frac{1}{n}+3 n \vartheta\right)<\frac{1}{n}+\frac{7}{2} n \vartheta
$$


(which follows from (15.4)) to conclude that

$$
\left\{\begin{aligned}
\prod_{\substack{w \in \mathfrak{M}(F) \\
w \mid v_{0}}} \max _{\substack{j_{11}, \ldots, j_{m n} \\
\leq}}\left(\| L_{1}^{(w)}\left(\boldsymbol{u}_{1}\right)^{j_{11}}\right. & \left.\ldots L_{n}^{(w)}\left(\boldsymbol{u}_{m}\right)^{j_{m n}} \|_{w}\right) \leq \\
\leq \prod_{\substack{w \in \mathfrak{M}(F) \\
w \mid v_{0}}}\left(\left(\left(\frac{2 n}{\vartheta}\right)^{s(w)}(1\right.\right. & \left.+\varepsilon)^{2 d\left(w / v_{0}\right)}\right)^{q} \times \\
& \left.\times Q_{1}^{r_{1}\left(\frac{m}{n}+\frac{7}{2} m n \vartheta\right)\left(b_{1 w}+\ldots+b_{n w}\right)-n \delta r_{1}\left(\frac{m}{n}-3 m n \vartheta\right) d\left(w / v_{0}\right)}\right) .
\end{aligned}\right.
$$

Now by $(15.10)-(15.12)$

$$
0 \leq-\sum_{\substack{w \in \mathfrak{M}(F) \\ w \nmid v_{0}}}\left(b_{1 w}+\ldots+b_{n w}\right)=\sum_{\substack{w \in \mathfrak{M}(F) \\ w \mid v_{0}}}\left(b_{1 w}+\ldots+b_{n w}\right) \leq n
$$

Therefore (15.21) and (15.22) in conjunction with (15.4) entail

$$
\left\{\begin{array}{l}
\prod_{w \in \mathfrak{M}(F)} \underset{j_{11}, \ldots, j_{m n}}{\max ^{*}}\left(\left\|L_{1}^{(w)}\left(\boldsymbol{u}_{1}\right)^{j_{11}} \ldots L_{n}^{(w)}\left(\boldsymbol{u}_{m}\right)^{j_{m n}}\right\|_{w}\right) \leq \\
\leq\left(\left(\frac{2 n}{\vartheta}\right)(1+\varepsilon)^{2}\right)^{q} Q_{1}^{\left.-m r_{1} \delta+3 m n^{2} r_{1} \vartheta+\frac{7}{2} m n^{2} r_{1} \vartheta+3 m n^{2} \vartheta r_{1} \delta\right)} \\
\quad \leq\left(\left(\frac{2 n}{\vartheta}\right)(1+\varepsilon)^{2}\right)^{q} Q_{1}^{\left.-\frac{1}{2} m r_{1} \delta\right)} .
\end{array}\right.
$$

We return to the sum in (15.17). By (14.2) it has $\leq 2^{q n}$ summands. Thus

$$
\begin{aligned}
& \left\|P^{\mathfrak{I}}\left(\boldsymbol{u}_{1}, \ldots, \boldsymbol{u}_{m}\right)\right\|_{w} \leq \\
& \quad \leq 2^{q n s(w)} \max _{j_{11}, \ldots, j_{m n}}\left\|d_{w}^{\mathfrak{I}}\left(j_{11}, \ldots, j_{m n}\right)\right\|_{w} \cdot \max _{j_{11}, \ldots, j_{m n}}\left(\left\|L_{1}^{(w)}\left(\boldsymbol{u}_{1}\right)^{j_{11}} \ldots L_{n}^{(w)}\left(\boldsymbol{u}_{m}\right)^{j_{m n}}\right\|_{w}\right) .
\end{aligned}
$$

So we get

$$
\begin{aligned}
& \prod_{w \in \mathfrak{M}(F)}\left\|P^{\mathfrak{I}}\left(\boldsymbol{u}_{1}, \ldots, \boldsymbol{u}_{m}\right)\right\|_{w} \leq \\
& \leq 2^{q n} \prod_{w \in \mathfrak{M}(F)} \max _{j_{11}, \ldots, j_{m n}}\left\|d_{w}^{\mathfrak{I}}\left(j_{11}, \ldots, j_{m n}\right)\right\|_{w} \times \\
& \quad \times \prod_{w \in \mathfrak{M}(F)} \max _{j_{11}, \ldots, j_{m n}}\left(\left\|L_{1}^{(w)}\left(\boldsymbol{u}_{1}\right)^{j_{11}} \ldots L_{n}^{(w)}\left(\boldsymbol{u}_{m}\right)^{j_{m n}}\right\|_{w}\right) .
\end{aligned}
$$

By (ii) of the Polynomial Theorem (Lemma 14.3)

$$
\begin{aligned}
& \prod_{w \in \mathfrak{M}(F)} \max _{j_{11}, \ldots, j_{m n}}\left(\left\|d_{w}^{\mathfrak{I}}\left(j_{11}, \ldots, j_{m n}\right)\right\|_{w}\right)= \\
& \quad=\prod_{v \in \mathfrak{M}(K)} \max _{j_{11}, \ldots, j_{m n}}\left(\left\|d_{v}^{\mathfrak{I}}\left(j_{11}, \ldots, j_{m n}\right)\right\|_{v}\right) \leq \mathcal{D}_{K}^{\frac{1}{2 d}}\left(2^{5 n+5} \mathcal{H}^{2}\right)^{q} .
\end{aligned}
$$

Combination of (15.23), (15.24), (15.25) yields

$$
\prod_{w \in \mathfrak{M}(F)}\left\|P^{\mathfrak{I}}\left(\boldsymbol{u}_{1}, \ldots, \boldsymbol{u}_{m}\right)\right\|_{w} \leq 2^{q n} \mathcal{D}_{K}^{\frac{1}{2 d}}\left(2^{5 n+5} \mathcal{H}^{2}\right)^{q}\left(\left(\frac{2 n}{\vartheta}\right)(1+\varepsilon)^{2}\right)^{q} Q_{1}^{-\frac{1}{2} m r_{1} \delta}
$$


We now use (15.5) and assume $1+\varepsilon<\sqrt{2}$ to conclude that

$$
\begin{aligned}
\prod_{w \in \mathfrak{M}(F)}\left\|P^{\mathfrak{I}}\left(\boldsymbol{u}_{1}, \ldots, \boldsymbol{u}_{m}\right)\right\|_{w} & \leq 2^{10 q n} \mathcal{D}_{K}^{\frac{1}{2 d}} \mathcal{H}^{2 q} \vartheta^{-q} Q_{1}^{-\frac{1}{2} m r_{1} \delta} \\
& \leq \prod_{h=1}^{m}\left(2^{10 n} \mathcal{D}_{K}^{\frac{1}{2 d q}} \mathcal{H}^{2} \vartheta^{-1} Q_{h}^{-\frac{1}{2} \delta(1+\vartheta)^{-1}}\right)^{r_{h}} .
\end{aligned}
$$

By (15.4) and (15.7) this is

$$
<\prod_{h=1}^{m}\left(2^{10 n} \mathcal{D}_{K}^{\frac{1}{2 d q}} \mathcal{H}^{2} \vartheta^{-1} Q_{h}^{-\frac{2}{5} \delta}\right)^{r_{h}}<1 .
$$

So (15.16) is established and the Lemma follows.

\section{The Penultimate Minimum}

Again we consider for $Q>1$ the parallelepipeds $\Pi(Q, \boldsymbol{c})$ defined with our forms $\left(L_{i}^{(v)}\right)$ $(v \in \mathfrak{M}(K), i=1, \ldots, n)$ and tuples $\left(c_{i v}\right)$ as in $(6.1)$ - (6.8). We write $\lambda_{1}(Q), \ldots, \lambda_{n}(Q)$ for the successive minima of $\Pi(Q, \boldsymbol{c})$.

Suppose $0<\varepsilon<1$ and let $\boldsymbol{g}_{1}(Q), \ldots, \boldsymbol{g}_{n-1}(Q)$ be linearly independent points in $\overline{\mathbb{Q}}^{n}$ with

$$
\boldsymbol{g}_{i}(Q) \in(1+\varepsilon) \lambda_{n-1}(Q) * \Pi(Q, \boldsymbol{c}) \quad(i=1, \ldots, n-1) .
$$

Write $T(Q)$ for the subspace of $\overline{\mathbb{Q}}^{n}$ generated by $\boldsymbol{g}_{1}(Q), \ldots, \boldsymbol{g}_{n-1}(Q)$.

Lemma 16.1 Let $0<\delta<1$. Suppose that

$$
m>1600 n^{4} \delta^{-2} \log 2 r .
$$

Put

$$
E=41 m^{2} n^{2} \delta^{-1}
$$

Suppose that

$$
\lambda_{n-1}=\lambda_{n-1}(Q)>Q^{-\delta}
$$

and

$$
Q^{\delta}>(2 \mathcal{H})^{3 R m^{3 m}}
$$

Assume that there does not exist a single proper subspace $T_{0}$ of $\overline{\mathbb{Q}}^{n}$ such that for all $Q$ with (16.4), (16.5) we have $T(Q)=T_{0}$.

Then the numbers $Q$ satisfying (16.4) and (16.5) are contained in the union of

$$
m-1
$$

intervals of type

$$
Q_{h}<Q \leq Q_{h}^{E} \quad(h=1, \ldots, m-1)
$$


Proof. We proceed along the same lines as Schmidt [14] (p. 159 ff.).

Suppose the Lemma were false.

Let $Q_{1}$ be the infimum of values $Q$ with (16.4) and (16.5). So $Q$ with (16.4), (16.5) will satisfy $Q>Q_{1}$. Now if all the values $Q$ with (16.4), (16.5) were in the interval $Q_{1}<Q \leq Q_{1}^{E}$ the Lemma was true. Therefore there are $Q>Q_{1}^{E}$ with (16.4). Let $Q_{2}$ be their infimum. And so forth.

In this way we will find numbers $Q_{1}, \ldots, Q_{m}$ with

$$
\lambda_{n-1}\left(Q_{h}\right) \leq Q_{h}^{-\delta} \quad(h=1, \ldots, m)
$$

and with

$$
Q_{h+1} \geq Q_{h}^{E} \quad(h=1, \ldots m-1) .
$$

Our goal is to apply on the one hand Lemma 15.1 and on the other hand Proposition 13.1. We will prove that under our hypotheses the two assertions contradict each other. We first check the hypotheses of Lemma 15.1. We put

$$
\vartheta=\frac{\delta}{20 n^{2}} \text {. }
$$

Then (15.4) is satisfied. Choose $r_{1}$ so large that

$$
r_{1}>\vartheta^{-1} \log Q_{m} / \log Q_{1}
$$

Given $r_{1}$, we put for $h=2, \ldots, m$

$$
r_{h}=\left[r_{1} \log Q_{1} / \log Q_{h}\right]+1 .
$$

In conjunction with (16.10) this implies

$$
r_{1} \log Q_{1} \leq r_{h} \log Q_{h} \leq(1+\vartheta) r_{1} \log Q_{1} \quad(h=1, \ldots, m),
$$

so that (15.5) is satisfied.

To guarantee (15.7), apart from (16.10) we require for $r_{1}$

$$
2^{r_{1}}>\mathcal{D}_{K}^{\frac{5}{4 d}}
$$

Since $q=r_{1}+\ldots+r_{m}$, we then have $\mathcal{D}_{K}^{5 / 4 d q}<2$ and (15.7) will be true provided

$$
Q_{1}^{\delta}>2^{25 n} \mathcal{H}^{5} \cdot 2 \cdot \delta^{-\frac{5}{2}}\left(20 n^{2}\right)^{5 / 2}
$$

Clearly, (16.2) and (16.5) amply imply (16.14).

Let $P$ be the approximation polynomial from section 14, constructed with respect to $L_{1}, \ldots, L_{r}$, with $\vartheta$ as in (16.9) and with $r_{1}, \ldots, r_{m}$ having (16.10) - (16.13). By (16.2) and (16.9), hypothesis (14.7) of the Index Theorem with $s=r$ is satisfied. Moreover, by 
Lemma 11.4 hypothesis (14.6) will be true with $H=n^{1 / 2} \mathcal{H}$.

So the approximation polynomial $P$ from the Index Theorem by Lemma 15.1 satisfies

$$
\text { ind } P\left(V_{[1]}\left(Q_{1}\right), \ldots, V_{[m]}\left(Q_{m}\right)\right) \geq m \vartheta \text {. }
$$

To derive an upper bound for this index we want to apply Proposition 13.1. Let us check its hypotheses.

Using (16.11), (16.12), (16.8), (16.9), (16.3) we see that

$$
\begin{aligned}
r_{h} \geq r_{1} \log Q_{1} / \log Q_{h} & \geq \frac{1}{1+\vartheta} r_{h+1} \log Q_{h+1} / \log Q_{h} \\
& \geq \frac{E}{1+\vartheta} r_{h+1} \geq \frac{41 m^{2} n^{2} \delta^{-1}}{1+\frac{\delta}{20 n^{2}}} r_{h+1} \geq \frac{2 m^{2}}{\vartheta} r_{h+1},
\end{aligned}
$$

and this is hypothesis (13.6).

As for (13.7), we first remark that by (16.4) and (16.5) the hypotheses of Lemma 12.4 are satisfied. By our assumption the second alternative in that Lemma is excluded.

Thus by the first alternative of Lemma 12.4 we obtain

$$
H\left(V\left(Q_{h}\right)\right)>Q_{h}^{\delta / 3 R} \quad(h=1, \ldots, m) .
$$

In conjunction with (16.12) this gives

$$
H\left(V\left(Q_{h}\right)\right)^{r_{h}}>Q_{1}^{\frac{\delta}{3 R} r_{1}} \quad(h=1, \ldots, m) .
$$

Therefore, to verify (13.7) is suffices to show that

$$
Q_{1}^{\frac{\delta}{3 R} r_{1}} \geq\left\{\exp \left(r_{1}+\ldots+r_{m}\right) H(P)\right\}^{(n-1)\left(\frac{3 m^{2}}{\vartheta}\right)^{m}} .
$$

By Lemma 14.2 with $H=n^{1 / 2} \mathcal{H}$ (cf. Lemma 11.4)

$$
H(P)<\mathcal{D}_{K}^{\frac{1}{2 d}} 2^{\frac{3}{2} m n}(6 n \mathcal{H})^{q} .
$$

By (16.16) and (16.9)

$$
\frac{r_{h}}{r_{1}} \leq \frac{\delta}{40 m^{2} n^{2}} \quad \text { for } h=2, \ldots, m
$$

Therefore

$$
\frac{q}{r_{1}}=\frac{r_{1}+\ldots+r_{m}}{r_{1}}<2 .
$$

To guarantee (16.18) it therefore suffices in view of (16.9) to guarantee that

$$
Q_{1}^{\delta / 3 R} \geq\left(e^{2} \mathcal{D}_{K}^{\frac{1}{2 d r_{1}}}(6 n \mathcal{H})^{2}\right)^{(n-1)\left(60 m^{2} n^{2} \delta^{-1}\right)^{m}} .
$$

However by (16.13) and (16.2) we get

$$
\left(e^{2} \mathcal{D}_{K}^{\frac{1}{2 d r_{1}}}(6 n \mathcal{H})^{2}\right)^{(n-1)\left(60 m^{2} n^{2} \delta^{-1}\right)^{m}}<(2 \mathcal{H})^{m^{3 m}} .
$$


Altogether we see that (16.5) implies (16.18). Thus by (16.17) hypothesis (13.7) is satisfied as well.

Now Proposition 13.1 says that

$$
\operatorname{ind}\left(V_{[1]}\left(Q_{1}\right), \ldots, V_{[m]}\left(Q_{m}\right) ; \boldsymbol{r}\right)<m \vartheta .
$$

This contradicts (16.15) and therefore the assertion of the Lemma follows.

Lemma 16.2 Suppose $0<\delta<1$. Let

$$
m>1600 n^{4} \delta^{-2} \log 2 r
$$

and put

$$
E=41 m^{2} n^{2} \delta^{-1}
$$

Given $Q>1$, let $T(Q)$ be the subspace generated by the points $\boldsymbol{g}_{1}(Q), \ldots, \boldsymbol{g}_{n-1}(Q)$ in (16.1).

Then there is a collection of proper linear subspaces $T_{1}, \ldots, T_{a}$ of $\overline{\mathbb{Q}}^{n}$ with

$$
a<m\left(1+\frac{5000}{999} \delta^{-1} \log E\right)
$$

and with the following property:

For any $Q$ satisfying

$$
\lambda_{n-1}(Q)<Q^{-\delta}
$$

and

$$
Q^{\delta}>(2 \mathcal{H})^{3 R m^{3 m}}
$$

the subspace $T(Q)$ will coincide with one of the subspaces $T_{1}, \ldots, T_{a}$.

Proof. By Lemma 16.1 we have two alternatives.

Either one single subspace will suffice and we are done.

Or the numbers $Q$ satisfying (16.23) and (16.24) are contained in the union of $m-1$ intervals

$$
Q_{h}<Q \leq Q_{h}^{E} \quad(h=1, \ldots, m-1) .
$$

For each interval (16.25) we may apply Lemma 8.1.

By (16.1) the points $\boldsymbol{g}_{1}, \ldots, \boldsymbol{g}_{n-1}$ lie in $(1+\varepsilon) Q^{-\delta} * \Pi(Q, \boldsymbol{c})$. Since $0<\varepsilon<1$ and by $(16.20)$ and $(16.24)$ they certainly lie in $Q^{-\frac{999}{1000} \delta} * \Pi(Q, \boldsymbol{c})$. So we apply Lemma 8.1 with $\frac{999}{1000} \delta$ instead of $\delta$. The analogue of (8.1) by (16.24) is amply satisfied. Consequently, each interval (16.25) gives rise to not more than

$$
1+\frac{5000}{999} \delta^{-1} \log E
$$

subspaces. Introducing a factor $m-1$ for the number of intervals we get the assertion in (16.22). 


\section{Approximating the Minima}

Lemma 17.1 Let $\Pi(Q, \boldsymbol{c})$ be the parallelepiped given by $(6.10)$, (6.11) with forms $\left(L_{i}^{(v)}\right)$ and tuples $\left(c_{i v}\right)$ satisfying $(6.1)$ - (6.8). Let $\lambda_{1}(Q)$ be the first minimum of $\Pi(Q, \boldsymbol{c})$. Suppose $\eta>0$ and

$$
Q^{\eta}>n^{3 / 2} \mathcal{H}
$$

Then we have

$$
\lambda_{1}(Q)>Q^{-1-\eta}
$$

Proof. Let $H_{Q, \boldsymbol{c}}(\boldsymbol{x})$ be the twisted height associated with $\Pi(Q, \boldsymbol{c})$. By Corollary 7.4 the minima of $H_{Q, \boldsymbol{c}}(\boldsymbol{x})$ and of $\Pi(Q, \boldsymbol{c})$ coincide. Therefore in order to prove (17.2), by (17.1) it suffices to show that for any $\boldsymbol{x} \in \overline{\mathbb{Q}}^{n}, \boldsymbol{x} \neq 0$

$$
H_{Q, \boldsymbol{c}}(\boldsymbol{x}) \geq n^{-3 / 2} \mathcal{H}^{-1} Q^{-1}
$$

Given $\boldsymbol{x} \in \overline{\mathbb{Q}}^{n}, x \neq 0$ let $F$ be a finite extension of $K$ with $\boldsymbol{x} \in F^{n}$.

Writing $\boldsymbol{x}=\left(x_{1}, \ldots, x_{n}\right)$ we have for $w \in \mathfrak{M}(F)$ and $i=1, \ldots, n$

$$
x_{i}=\gamma_{i 1}^{(w)} L_{1}^{(w)}(\boldsymbol{x})+\ldots+\gamma_{i n}^{(w)} L_{n}^{(w)}(\boldsymbol{x}) .
$$

Here the $\gamma_{i j}^{(w)}$-s are the entries of the matrix which is inverse to the coefficient matrix of $L_{1}^{(w)}, \ldots, L_{n}^{(w)}$.

Lemma 11.6 says that

$$
\prod_{w \in \mathfrak{M}(F)} \max _{1 \leq i, j \leq n}\left\|\gamma_{i j}^{(w)}\right\|_{w} \leq \mathcal{H}
$$

We infer from (17.4) and (17.5) that

$$
\begin{aligned}
H(\boldsymbol{x}) & \leq n^{3 / 2}\left(\prod_{w \in \mathfrak{M}(F)} \max _{1 \leq i, j \leq n}\left\|\gamma_{i j}^{(w)}\right\|_{w}\right)\left(\prod_{w \in \mathfrak{M}(F)} \max _{1 \leq i \leq n}\left\|L_{i}^{(w)}(\boldsymbol{x})\right\|_{w}\right) \\
& \leq n^{3 / 2} \mathcal{H} \prod_{w \in \mathfrak{M}(F)} \max _{1 \leq i \leq n}\left\|L_{i}^{(w)}(\boldsymbol{x})\right\|_{w} .
\end{aligned}
$$

Since $H(\boldsymbol{x}) \geq 1$ this implies

$$
\prod_{w \in \mathfrak{M}(F)} \max _{1 \leq i \leq n}\left\|L_{i}^{(w)}(\boldsymbol{x})\right\|_{w} \geq n^{-3 / 2} \mathcal{H}^{-1} .
$$

On the other hand, by (2.12), (6.4), (6.5)

$$
H_{Q, \boldsymbol{c}}(\boldsymbol{x})=\prod_{w \in \mathfrak{M}(F)} \max _{1 \leq i \leq n} \frac{\left\|L_{i}^{(w)}(\boldsymbol{x})\right\|_{w}}{Q^{c_{i w}}} \geq Q^{-1} \prod_{w \in \mathfrak{M}(F)} \max _{1 \leq i \leq n}\left\|L_{i}^{(w)}(\boldsymbol{x})\right\|_{w} .
$$


Together with (17.6) this gives

$$
H_{Q, \boldsymbol{c}}(\boldsymbol{x}) \geq n^{-3 / 2} \mathcal{H}^{-1} Q^{-1}
$$

and (17.3) is verified.

Lemma 17.2 Let $B$ be a natural number. Let $\mathcal{P}$ be the set of $Q>1$ such that

$$
Q^{1 / B}>\max \left\{n^{3 / 2} \mathcal{H}, 2^{\frac{n(n-1)}{2}}\right\}
$$

For $Q \in \mathcal{P}$ write $\lambda_{1}(Q), \ldots, \lambda_{n}(Q)$ for the successive minima of $\Pi(Q, \boldsymbol{c})$.

Then there exists a finite set $\mathcal{K}$ of tuples of real numbers $\left(\gamma_{1}, \ldots, \gamma_{n}\right)$ of cardinality

$$
\operatorname{card} \mathcal{K} \leq(n(B+1)+1)^{n}
$$

with the following property:

The elements $\gamma \in \mathcal{K}$ satisfy

$$
-1 \leq \gamma_{i} \leq n-1+\frac{n}{B} \quad(i=1, \ldots, n) .
$$

Moreover, for any $Q \in \mathcal{P}$ and for any permutation $\varphi$ of $\{1, \ldots, n\}$ there exists $\gamma \in \mathcal{K}$ with

$$
Q^{\gamma_{i}-\frac{1}{B}}<\lambda_{\varphi(i)}(Q) \leq Q^{\gamma_{i}} \quad(i=1, \ldots, n)
$$

Proof. By Corollary 7.6 we have

$$
n^{-\frac{n}{2}} \leq \lambda_{1}(Q) \ldots \lambda_{n}(Q) \leq 2^{\frac{n(n-1)}{2}} .
$$

Notice that in view of (17.7), hypothesis (17.1) of Lemma 17.1 is satisfied with $\eta=\frac{1}{B}$. Consequently

$$
\lambda_{1}(Q)>Q^{-1-\frac{1}{B}}
$$

and thus by (17.11), (17.7)

$$
\lambda_{n}(Q)<Q^{n-1+\frac{n}{B}}
$$

Taking for $\gamma$ the points with components $\gamma_{i}$ of the shape $\frac{l_{i}}{B}$ with $l_{i} \in \mathbb{Z}$ and such that $l_{i} \in[-B,(n-1) B+n]$, in view of $(17.12),(17.13)$ we clearly can find for any pair $Q, \varphi$ under consideration a suitable $\gamma$ satisfying (17.9), (17.10). The number of such points equals $(n(B+1)+1)^{n}$ and so (17.8) is true as well. 


\section{Two Adjacent Minima}

We go back to the situation studied in section 16 .

So we have the parallelepiped $\Pi(Q, \boldsymbol{c})$ with forms $L_{i}^{(v)}$ and a tuple $\boldsymbol{c}=\left(c_{i v}\right)$ $(v \in \mathfrak{M}(K), i=1, \ldots, n)$ as in $(6.1)-(6.8) . \lambda_{1}(Q), \ldots, \lambda_{n}(Q)$ are the successive minima of $\Pi(Q, \boldsymbol{c})$ and $\boldsymbol{g}_{1}(Q), \ldots, \boldsymbol{g}_{n}(Q)$ are linearly independent points in $\overline{\mathbb{Q}}^{n}$ with

$$
\boldsymbol{g}_{i}(Q) \in(1+\varepsilon) \lambda_{i}(Q) * \Pi(Q, \boldsymbol{c}) \quad(i=1, \ldots, n) .
$$

We suppose that $\varepsilon$ is a small positive parameter with $\varepsilon<1$ and such that (9.14) is satisfied. Then we may apply Lemma 9.2. Consequently for each $Q$ we can find a permutation $\pi$ of $\{1, \ldots, n\}$ and points $\boldsymbol{h}_{1}(Q), \ldots, \boldsymbol{h}_{n}(Q)$ such that (9.18) and (9.19) are true, i.e., such that

$$
\operatorname{span}\left\{\boldsymbol{g}_{1}(Q), \ldots \boldsymbol{g}_{j}(Q)\right\}=\operatorname{span}\left\{\boldsymbol{h}_{1}(Q), \ldots, \boldsymbol{h}_{j}(Q)\right\} \quad(j=1, \ldots, n)
$$

and

$$
\left\{\begin{array}{c}
\left\|L_{i}^{(u)}\left(\boldsymbol{h}_{j}(Q)\right)\right\|_{u} \leq Q^{c_{i u}} \quad\left(u \in \mathfrak{M}(E), u \nmid v_{0}, 1 \leq i, j \leq n\right) \\
\left\|L_{\pi(i)}^{(u)}\left(\boldsymbol{h}_{j}(Q)\right)\right\|_{u}=\left\|h_{j \pi(i)}(Q)\right\|_{u} \leq\left((1+\varepsilon)^{n+1} 2^{n^{2}} \min \left\{\lambda_{i}(Q), \lambda_{j}(Q)\right\}\right)^{d\left(u / v_{0}\right)} \\
\left(u \in \mathfrak{M}(E), u \mid v_{0}, 1 \leq i, j \leq n\right) .
\end{array}\right.
$$

Now let $1 \leq k \leq n-1$. We apply Lemma 10.1 with

$$
\mu_{i}=\mu_{i}(Q)=(1+\varepsilon)^{n+1} 2^{n^{2}} \lambda_{i}(Q) \quad(i=1, \ldots, n) .
$$

For $\sigma=\left\{1 \leq i_{1}<\ldots<i_{k} \leq n\right\} \in C(n, k)$ we define $\mu_{\sigma}=\mu_{\sigma}(Q)$ as in (10.6), i.e. $\mu_{\sigma}=\mu_{i_{1}} \cdots \mu_{i_{k}}$.

With $N=\left(\begin{array}{l}n \\ k\end{array}\right)$ we let $\tau_{1}, \ldots, \tau_{N}$ be the enumeration of $C(n, k)$ in the lexicographical ordering. Notice that by (18.4) we have

$$
\mu_{1} \leq \ldots \leq \mu_{n}
$$

Therefore,

$$
\mu_{\tau_{j}} \leq \mu_{\tau_{N-1}} \leq \mu_{\tau_{N}} \quad \text { for } j=1, \ldots N-2
$$

(however, in general we do not get $\mu_{\tau_{1}} \leq \mu_{\tau_{2}} \leq \ldots \leq \mu_{\tau_{N-2}}$ ).

We combine (18.2), (18.3) and Lemma 10.1 to obtain:

There exist a finite extension $F$ of $E$ and an element $\beta \in F^{*}$ such that

$$
\boldsymbol{h}_{1}(Q), \ldots, \boldsymbol{h}_{n}(Q) \in F^{n}
$$

and such that moreover

$$
\operatorname{span}\left\{\boldsymbol{g}_{1}(Q), \ldots \boldsymbol{g}_{j}(Q)\right\}=\operatorname{span}\left\{\boldsymbol{h}_{1}(Q), \ldots, \boldsymbol{h}_{j}(Q)\right\} \quad(j=1, \ldots, n)
$$




$$
\left\{\begin{array}{r}
\left\|L_{\tau_{i}}^{(w)}\left(\beta \boldsymbol{h}_{\tau_{j}}(Q)\right)\right\|_{w} \leq Q^{c_{\tau_{i} w}} \quad\left(w \in \mathfrak{M}(F), w \nmid v_{0}, 1 \leq i, j \leq N\right) \\
\left\|L_{\pi \tau_{i}}^{(w)}\left(\beta \boldsymbol{h}_{\tau_{j}}(Q)\right)\right\|_{w}=\left\|\beta h_{\tau_{j}, \pi \tau_{i}}(Q)\right\|_{w} \leq\left(k^{k} \min \left\{\mu_{\tau_{i}}, \mu_{\tau_{j}}\right\}\right)^{d\left(w / v_{0}\right)} \\
\left(w \in \mathfrak{M}(F), w \mid v_{0}, 1 \leq i, j \leq N\right) .
\end{array}\right.
$$

(Here we have written $\boldsymbol{h}_{\tau_{j}}=\left(h_{\tau_{j}, \tau_{1}}, \ldots, h_{\tau_{j}, \tau_{N}}\right)$.)

We now derive for the points $\boldsymbol{h}_{\tau_{1}}, \ldots, \boldsymbol{h}_{\tau_{N-1}}$ inequalities which avoid the minimum we have in (18.7) and which again are related to parallelepipeds. For $\sigma=\left\{i_{1}<\ldots<i_{k}\right\} \in C(n, k)$ we write

$$
\lambda_{\sigma}(Q)=\lambda_{i_{1}}(Q) \cdots \lambda_{i_{k}}(Q) .
$$

Notice that for the $\lambda_{\sigma}(Q)$-s we have inequalities similar to (18.5).

Lemma 18.1 Suppose $1 \leq k \leq n$. Let $B \geq n$ be an integer. We can cover the set of numbers $Q$ with

$$
Q^{1 / B} \geq 2^{2 n^{3}} \mathcal{H}
$$

by subsets $\mathcal{A}_{1}, \ldots, \mathcal{A}_{G}$ with

$$
G \leq(2 n B)^{n} .
$$

For each $g(1 \leq g \leq G)$ there exist real numbers $e_{1 g}, \ldots, e_{N g}$ with

$$
e_{1 g}+\ldots+e_{N g}=0 \quad \text { and } \quad\left|e_{i g}\right| \leq 2 k n \quad(i=1, \ldots, N)
$$

such that the following assertion is true.

Let $\tau_{1}, \ldots, \tau_{N}$ be the lexicographical ordering of $C(n, k)$. Then for each $Q$ in $\mathcal{A}_{g}$ the points $\boldsymbol{h}_{\tau_{1}}(Q), \ldots, \boldsymbol{h}_{\tau_{N-1}}(Q)$ satisfy

$$
\begin{aligned}
\left\|L_{\tau_{i}}^{(w)}\left(\beta \boldsymbol{h}_{\tau_{j}}(Q)\right)\right\|_{w} \leq & Q^{c_{\tau_{i} w}} \quad\left(w \in \mathfrak{M}(F), w \nmid v_{0}, 1 \leq i \leq N, 1 \leq j \leq N-1\right) \\
\left\|L_{\tau_{i}}^{(w)}\left(\beta \boldsymbol{h}_{\tau_{j}}(Q)\right)\right\|_{w} \leq & \left(Q^{e_{i g}+\frac{n}{B}}\left\{\lambda_{\tau_{N-1}}(Q) / \lambda_{\tau_{N}}(Q)\right\}^{1 / N}\right)^{d\left(w / v_{0}\right)} \\
& \left(w \in \mathfrak{M}(F), w \mid v_{0}, 1 \leq i \leq N, 1 \leq j \leq N-1\right) .
\end{aligned}
$$

Proof. We first notice that (18.11) is an immediate consequence of the first part of (18.7). As for (18.12), for a permutation $\pi$ of $\{1, \ldots, n\}$ we write $\varphi$ for the inverse permutation. Then from the second part of (18.7) we get by (18.5), ignoring the inequality for $j=N$,

$$
\begin{aligned}
\left\|L_{\tau_{i}}^{(w)}\left(\beta \boldsymbol{h}_{\tau_{j}}(Q)\right)\right\|_{w} \leq & \left(k^{k} \min \left\{\mu_{\varphi \tau_{i}}, \mu_{\tau_{N-1}}\right\}\right)^{d\left(w / v_{0}\right)} \\
& \left(w \in \mathfrak{M}(F), w \mid v_{0}, 1 \leq i \leq N, 1 \leq j \leq N-1\right) .
\end{aligned}
$$

We now cover the set of pairs $(\varphi, Q)\left(\varphi \in \mathfrak{S}_{n}, Q\right.$ satisfying (18.8)) by subsets as follows. Two pairs $\left(\varphi_{1}, Q_{1}\right)$ and $\left(\varphi_{2}, Q_{2}\right)$ will belong to the same subset if they give rise to the 
same $n$-tuple $\gamma=\left(\gamma_{1}, \ldots, \gamma_{n}\right)$ in Lemma 17.2. By (17.8) and since $B \geq 2$ the number of subsets is

$$
\leq(2 n B)^{n}
$$

Fix such a subset $\mathfrak{B}$, say. Then for any pair $(\varphi, Q) \in \mathfrak{B}$ we have

$$
Q^{\gamma_{i}-\frac{1}{B}}<\lambda_{\varphi(i)}(Q) \leq Q^{\gamma_{i}} \quad(i=1, \ldots, n)
$$

where $\gamma=\left(\gamma_{1}, \ldots, \gamma_{n}\right)$ is fixed and satisfies

$$
-1 \leq \gamma_{i} \leq n-1+\frac{n}{B} \quad(i=1, \ldots, n) .
$$

For $\sigma=\left\{1 \leq i_{1}<\ldots<i_{k} \leq n\right\} \in C(n, k)$ we define

$$
\gamma_{\sigma}=\gamma_{i_{1}}+\ldots+\gamma_{i_{k}}
$$

and

$$
\lambda_{\varphi \sigma}(Q)=\lambda_{\varphi\left(i_{1}\right)}(Q) \ldots \lambda_{\varphi\left(i_{k}\right)}(Q) .
$$

Then (18.15) implies

$$
Q^{\gamma_{\sigma}-\frac{k}{B}}<\lambda_{\varphi \sigma}(Q) \leq Q^{\gamma_{\sigma}} \quad(\sigma \in C(n, k))
$$

Let $\sigma_{1}, \ldots, \sigma_{N}$ be an enumeration of $C(n, k)$ such that

$$
\gamma_{\sigma_{1}} \leq \ldots \leq \gamma_{\sigma_{N}}
$$

In analogy with (18.5) we have

$$
\lambda_{\tau_{j}}(Q) \leq \lambda_{\tau_{N-1}}(Q) \leq \lambda_{\tau_{N}}(Q) \text { for } j=1, \ldots N-2 .
$$

We may conclude that

$$
Q^{\gamma_{\sigma_{N-1}}-\frac{k}{B}}<\lambda_{\tau_{N-1}}(Q) \leq Q^{\gamma_{\sigma_{N-1}}}
$$

For suppose (18.20) was wrong. If $\lambda_{\tau_{N-1}}(Q)>Q^{\gamma_{\sigma_{N-1}}}$ then by (18.17), (18.18) we get $\tau_{N-1}=\varphi \sigma_{N}$ and so $\tau_{N}=\varphi \sigma_{j}$ for some $j<N$. Hence $\lambda_{\tau_{N-1}}(Q)>Q^{\gamma_{\sigma_{j}}} \geq \lambda_{\tau_{N}}(Q)$. This contradicts (18.19). If however $\lambda_{\tau_{N-1}}(Q) \leq Q^{\gamma_{\sigma_{N-1}}-\frac{k}{B}}$ then in view of (18.17), (18.18) we have $\tau_{N-1}=\varphi \sigma_{k}$ for some $k<N-1$. It follows that there exists a pair $(j, i)$ with $j<N-1, i \geq N-1$ and $\tau_{j}=\varphi \sigma_{i}$. Consequently $\lambda_{\tau_{N-1}}(Q) \leq Q^{\gamma_{\sigma_{i}}-\frac{k}{B}}<\lambda_{\tau_{j}}(Q)$. Again this contradicts (18.19).

Combining (18.13), (18.4), (18.17), (18.20) we may infer that

$$
\left\{\begin{aligned}
\left\|L_{\tau_{i}}^{(w)}\left(\beta \boldsymbol{h}_{\tau_{j}}(Q)\right)\right\|_{w} \leq & \left(\left(k(1+\varepsilon)^{n+1} 2^{n^{2}}\right)^{k} \min \left\{\lambda_{\varphi \tau_{i}}(Q), \lambda_{\tau_{N-1}}(Q)\right\}\right)^{d\left(w / v_{0}\right)} \\
\leq & \left(\left(k(1+\varepsilon)^{n+1} 2^{n^{2}}\right)^{k} \min \left\{Q^{\gamma_{\tau_{i}}}, Q^{\gamma_{\sigma_{N-1}}}\right\}\right)^{d\left(w / v_{0}\right)} \\
& \left(w \in \mathfrak{M}(F), w \mid v_{0}, 1 \leq i \leq N, 1 \leq j \leq N-1\right) .
\end{aligned}\right.
$$


Notice that given $\gamma$ the exponents $\gamma_{\tau_{i}}(i=1, \ldots, N)$ and $\gamma_{\sigma_{N-1}}$ are uniquely determined. Write

$$
f_{i}=\min \left\{\gamma_{\tau_{i}}, \gamma_{\sigma_{N-1}}\right\} \quad(i=1, \ldots, N) .
$$

We observe that by (18.18) the exponents $f_{1}, \ldots, f_{N}$ are equal to $\gamma_{\sigma_{1}}, \ldots, \gamma_{\sigma_{N-1}}, \gamma_{\sigma_{N-1}}$ in a suitable order which depends only on $\boldsymbol{\gamma}$. Moreover we obtain in view of $(18.17),(18.20)$

$$
Q^{f_{1}+\ldots+f_{N}-N \frac{k}{B}}<\lambda_{\tau_{1}} \ldots \lambda_{\tau_{N-1}} \lambda_{\tau_{N-1}} \leq Q^{f_{1}+\ldots+f_{N}}
$$

(where we have written $\lambda_{\sigma}$ for $\lambda_{\sigma}(Q)$ ). On the other hand

$$
\lambda_{\tau_{1}} \ldots \lambda_{\tau_{N-1}} \lambda_{\tau_{N-1}}=\left(\lambda_{\tau_{N-1}} / \lambda_{\tau_{N}}\right)\left(\lambda_{\tau_{1}} \ldots \lambda_{\tau_{N}}\right)
$$

and

$$
\lambda_{\tau_{1}} \ldots \lambda_{\tau_{N}}=\left(\lambda_{1} \ldots \lambda_{n}\right)^{N \frac{n}{k}}
$$

So by Corollary 7.6

$$
n^{-N \frac{n^{2}}{2 k}}\left(\lambda_{\tau_{N-1}} / \lambda_{\tau_{N}}\right) \leq \lambda_{\tau_{1}} \ldots \lambda_{\tau_{N-1}} \lambda_{\tau_{N-1}} \leq 2^{N \frac{n^{2}(n-1)}{2 k}}\left(\lambda_{\tau_{N-1}} / \lambda_{\tau_{N}}\right) .
$$

We define exponents $e_{1}, \ldots, e_{N}$ by

$$
e_{i}=f_{i}-\frac{1}{N}\left(f_{1}+\ldots+f_{N}\right) \quad(i=1, \ldots, N)
$$

Combination of (18.23) and (18.24) yields

$$
\begin{aligned}
Q^{f_{i}} & \leq Q^{e_{i}+\frac{k}{B}}\left(\lambda_{\tau_{1}} \ldots \lambda_{\tau_{N-1}} \lambda_{\tau_{N-1}}\right)^{1 / N} \leq \\
& \leq Q^{e_{i}+\frac{k}{B}} 2^{\frac{n^{2}(n-1)}{2}}\left(\lambda_{\tau_{N-1}} / \lambda_{\tau_{N}}\right)^{1 / N} .
\end{aligned}
$$

Moreover, by (18.8) and since $\varepsilon<1$

$$
Q^{1 / B}>\left(k(1+\varepsilon)^{n+1} 2^{n^{2}}\right)^{k} 2^{\frac{n^{2}(n-1)}{2}} .
$$

Altogether (18.21) and (18.26) in view of $k \leq n-1$ imply

$$
\begin{aligned}
\left\|L_{\tau_{i}}^{(w)}\left(\beta \boldsymbol{h}_{\tau_{j}}(Q)\right)\right\|_{w}< & \left(Q^{e_{i}+\frac{n}{B}}\left(\lambda_{\tau_{N-1}} / \lambda_{\tau_{N}}\right)^{1 / N}\right)^{d\left(w / v_{0}\right)} \\
& \left(w \in \mathfrak{M}(F), w \mid v_{0} ; i=1, \ldots, N ; j=1, \ldots, N-1\right)
\end{aligned}
$$

and this is the desired $(18.12)$ for pairs $(\varphi, Q)$ in the particular set $\mathfrak{B}$. Clearly the covering of the set of pairs $(\varphi, Q)$ induces a covering of the set of $\mathrm{Q}$ with (18.8). The number of sets $\mathfrak{B}$ needed is bounded by (18.14) and so we get (18.9).

We still have to verify (18.10). (18.25) implies at once $e_{1}+\ldots+e_{N}=0$. Moreover by (18.16) and since $B \geq n$

$$
\left|\gamma_{\sigma}\right| \leq k(n-1)+\frac{k n}{B} \leq k n
$$


Together with (18.22), (18.25) this gives

$$
\left|e_{i}\right| \leq 2 k n
$$

and the Lemma follows.

We fix $g(1 \leq g \leq G)$ and we study parameters $Q \in \mathcal{A}_{g}$. Our goal is to apply the material from section 16 to inequalities (18.11), (18.12), more precisely to the parallelepiped defined by the inequalities

$$
\begin{array}{ll}
\left\|L_{\tau_{i}}^{(w)}(\boldsymbol{x})\right\|_{w} \leq Q^{c_{\tau_{i} w}} & \left(w \in \mathfrak{M}(F), w \nmid v_{0}, 1 \leq i \leq N\right) \\
\left\|L_{\tau_{i}}^{(w)}(\boldsymbol{x})\right\|_{w} \leq Q^{e_{i g} d\left(w / v_{0}\right)} & \left(w \in \mathfrak{M}(F), w \mid v_{0}, 1 \leq i \leq N\right)
\end{array}
$$

where $g$ is fixed and where $\boldsymbol{x} \in \overline{\mathbb{Q}}^{N}$.

The parallelepiped $\Pi(Q, \boldsymbol{c})$ studied in section 16 has $c_{1 v_{0}}=\ldots=c_{n v_{0}}=0$ (cf. (6.7), (6.12)). The analogue of this for (18.27) would be $e_{1 g}=\ldots=e_{N g}=0$. However in general this will not be true.

Lemma 18.2 Let $A_{g}$ be one of the sets in Lemma 18.1. Let $\varepsilon>0$. Fix a place $v_{1} \in \mathfrak{M}_{1} \backslash\left\{v_{0}\right\}$. Then for any $Q \in \mathcal{A}_{g}$ there exists a finite extension $F$ of $K$ and an element $\gamma \in F^{*}$ with the following properties:

The points $\boldsymbol{h}_{\tau_{1}}(Q), \ldots, \boldsymbol{h}_{\tau_{N-1}}(Q)$ from Lemma 18.1 lie in $F^{N}$ and satisfy the inequalities

$$
\left\{\begin{aligned}
\left\|L_{\tau_{i}}^{(w)}\left(\gamma \boldsymbol{h}_{\tau_{j}}(Q)\right)\right\|_{w} & \leq Q^{c_{\tau_{i} w}} \\
(w & \left.\in \mathfrak{M}(F), w \nmid v_{0}, w \nmid v_{1}, 1 \leq i \leq N, 1 \leq j \leq N-1\right) \\
\left\|L_{\tau_{i}}^{(w)}\left(\gamma \boldsymbol{h}_{\tau_{j}}(Q)\right)\right\|_{w} & \leq Q^{e_{i g} d\left(w / v_{0}\right)} \\
(w & \left.\in \mathfrak{M}(F), w \mid v_{0}, 1 \leq i \leq N, 1 \leq j \leq N-1\right) \\
\left\|L_{\tau_{i}}^{(w)}\left(\gamma \boldsymbol{h}_{\tau_{j}}(Q)\right)\right\|_{w} & =\left\|\gamma h_{\tau_{j}, \tau_{i}}(Q)\right\|_{w} \leq\left\{(1+\varepsilon) Q^{\frac{n}{B}}\left(\lambda_{\tau_{N-1}} / \lambda_{\tau_{N}}\right)^{1 / N}\right\}^{d\left(w / v_{1}\right)} \\
(w & \left.\in \mathfrak{M}(F), w \mid v_{1}, 1 \leq i \leq N, 1 \leq j \leq N-1\right) .
\end{aligned}\right.
$$

Proof. We apply Lemma 6.3 with $F$ replaced by $K$. We put

$$
\left\{\begin{array}{l}
A_{v}=1 \text { for } v \in \mathfrak{M}(K) \backslash\left\{v_{0}, v_{1}\right\} \\
A_{v_{0}}=Q^{-\frac{n}{B}}\left(\lambda_{\tau_{N-1}} / \lambda_{\tau_{N}}\right)^{-1 / N} \\
A_{v_{1}}=(1+\varepsilon) Q^{\frac{n}{B}}\left(\lambda_{\tau_{N-1}} / \lambda_{\tau_{N}}\right)^{1 / N}
\end{array}\right.
$$

Then obviously the hypotheses of Lemma 6.3 are satisfied. Accordingly there exists a finite extension $F$ of $K$ and an element $\alpha \in F^{*}$ satisfying

$$
\|\alpha\|_{w} \leq A_{v}^{d(w / v)} \quad \text { for } w \in \mathfrak{M}(F), w \mid v, v \in \mathfrak{M}(K)
$$


We may assume without loss of generality that the field $F$ in Lemma 18.1 we start with is large enough to deal with (18.30). ( $F$ naturally depends upon the particular $Q$ under consideration).

Notice that by $(6.7) c_{1 v_{1}}=\ldots=c_{n v_{n}}=0$, so that $c_{\tau_{1} v_{1}}=\ldots=c_{\tau_{N} v_{1}}=0$. Assertion (18.28) now follows directly from (18.11), (18.12) with $\gamma=\alpha \beta$.

For $Q \geq 1$ we define the parallelepiped $\Pi^{(k)}(Q, \boldsymbol{f})$ as follows: $\boldsymbol{f}=\left(f_{\tau_{i} v}\right)(v \in \mathfrak{M}(K), i=1, \ldots, N)$ is the tuple given by

$$
f_{\tau_{i} v}= \begin{cases}\frac{1}{2 n^{2}} c_{\tau_{i} v} & \text { for } v \in \mathfrak{M}(K), v \neq v_{0}, i=1, \ldots, n \\ \frac{1}{2 n^{2}} e_{i g} & \text { for } v=v_{0}\end{cases}
$$

If $\boldsymbol{x} \in \overline{\mathbb{Q}}^{N}$ and if $F$ is a finite extension of $K$ such that $\boldsymbol{x} \in F^{n}$, then $\boldsymbol{x}$ will belong to $\Pi^{(k)}(Q, \boldsymbol{f})$ if and only if it satisfies the inequalities

$$
\left\|L_{\tau_{i}}^{(w)}(\boldsymbol{x})\right\|_{w} \leq Q^{f_{\tau_{i} w}} \quad(w \in \mathfrak{M}(F), i=1, \ldots, N),
$$

where $f_{\tau_{i} w}=d(w / v) f_{\tau_{i} v}$ for $w \mid v, v \in \mathfrak{M}(K)$ and for $i=1, \ldots, N$.

For $\lambda>0$ we define $\lambda * \Pi^{(k)}(Q, \boldsymbol{f})$ as follows:

Suppose $\boldsymbol{x} \in \overline{\mathbb{Q}}^{N}$, indeed assume that $\boldsymbol{x} \in F^{N}$, where $F$ is as above. Then $\boldsymbol{x}$ lies in $\lambda * \Pi^{(k)}(Q, \boldsymbol{f})$ if and only if it satisfies the inequalities

$$
\begin{array}{r}
\left\|L_{\tau_{i}}^{(w)}(\boldsymbol{x})\right\|_{w} \leq Q^{f_{\tau_{i} w}} \quad\left(w \in \mathfrak{M}(F), w \nmid v_{1}, i=1, \ldots, N\right) \\
\left\|L_{\tau_{i}}^{(w)}(\boldsymbol{x})\right\|_{w}=\left\|x_{i}\right\|_{w} \leq Q^{f_{\tau_{i} w}} \lambda^{d\left(w / v_{1}\right)}=\lambda^{d\left(w / v_{1}\right)} \\
\left(w \in \mathfrak{M}(F), w \mid v_{1}, i=1, \ldots, N\right) .
\end{array}
$$

The main difference between our definition (6.13), (6.14) and (18.33), (18.34) is that we have now "concentrated" the factor $\lambda$ to the places $w$ lying above $v_{1}$ (instead of $v_{0}$ as in section 6).

We write $\mathfrak{M}_{2}=\mathfrak{M}_{1} \backslash\left\{v_{0}\right\}$. Then by (6.7) we may infer that for $v \in \mathfrak{M}_{2}$

$$
L_{\tau_{1}}^{(v)}=X_{1}, \ldots, L_{\tau_{N}}^{(v)}=X_{N} ; \quad f_{\tau_{1} v}=\ldots=f_{\tau_{N} v}=0 .
$$

Moreover since

$$
\operatorname{det}\left(L_{\tau_{1}}^{(v)}, \ldots, L_{\tau_{N}}^{(v)}\right)=\operatorname{det}\left(L_{1}^{(v)}, \ldots, L_{n}^{(v)}\right)^{\frac{k}{n} N}
$$

(6.4) implies that

$$
\operatorname{det}\left(L_{\tau_{1}}^{(v)}, \ldots, L_{\tau_{N}}^{(v)}\right)=1 \quad \text { for each } v \in \mathfrak{M}(K)
$$


Combination of (6.5), (18.10) and (18.31) implies

$$
\sum_{v \in \mathfrak{M}(K)} \sum_{i=1}^{N} f_{\tau_{i} v}=0 .
$$

Moreover, again by (6.5), (18.10)

$$
\sum_{\substack{v \in \mathfrak{M}(K) \\ v \neq v_{0}}} \max _{\substack{1 \leq i \leq N \\ \tau_{i} v}}+\sum_{i=1}^{N} e_{i g} \leq k+2 k n \leq 2 n^{2} .
$$

In conjunction with (18.31) this gives

$$
\sum_{v \in \mathfrak{M}(K)} \max _{1 \leq i \leq N} f_{\tau_{i} v} \leq 1
$$

Therefore, if we replace the family of forms $\left\{L_{1}, \ldots, L_{r}\right\}$ we start with in section 6 by the family of forms $\left\{L_{\sigma}\right\}=\left\{L_{i_{1}} \wedge \ldots \wedge L_{i_{k}}\right\}$ where $\sigma=\left\{i_{1}<\ldots<i_{k}\right\}$ runs through the $k$-element subsets of $\{1, \ldots, r\}$, we get a setting that is completely analogous to the one at the beginning of section 6 .

The differences are as follows:

$$
\left\{\begin{array}{lll}
r & \text { is replaced by } & \left(\begin{array}{l}
r \\
k
\end{array}\right) \\
n & \text { is replaced by } \quad N=\left(\begin{array}{l}
n \\
k
\end{array}\right) \\
\mathfrak{M}_{1} & \text { is replaced by } & \mathfrak{M}_{2} \\
v_{0} & \text { is replaced by } & v_{1} .
\end{array}\right.
$$

However, if at the beginning we have $R$ different systems $\left\{L_{1}^{(v)}, \ldots, L_{n}^{(v)}\right\}$, then in the definition of $\Pi^{(k)}(Q, \boldsymbol{f})$ again we have $R$ different systems $\left\{L_{\tau_{1}}^{(v)}, \ldots, L_{\tau_{n}}^{(v)}\right\}$.

Assertion (18.28) may now be reinterpreted in terms of the parallelepiped $\Pi^{(k)}\left(Q^{2 n^{2}}, \boldsymbol{f}\right)$ : For each $Q$ in $\mathcal{A}_{g}$ the points $\gamma \boldsymbol{h}_{\tau_{1}}(Q), \ldots, \gamma \boldsymbol{h}_{\tau_{N-1}}(Q)$ satisfy the relations

$$
\boldsymbol{h}_{\tau_{i}}(Q) \in\left\{(1+\varepsilon) Q^{\frac{n}{B}}\left(\lambda_{\tau_{N-1}}(Q) / \lambda_{\tau_{N}}(Q)\right)^{1 / N}\right\} * \Pi^{(k)}\left(Q^{2 n^{2}}, \boldsymbol{f}\right)
$$

$(i=1, \ldots, N-1)$.

Let $\nu_{1}(Q), \ldots, \nu_{N}(Q)$ be the successive minima of $\Pi^{(k)}(Q, \boldsymbol{f})$. Then (18.41) implies

$$
\nu_{N-1}\left(Q^{2 n^{2}}\right) \leq(1+\varepsilon) Q^{\frac{n}{B}}\left(\lambda_{\tau_{N-1}}(Q) / \lambda_{\tau_{N}}(Q)\right)^{1 / N} .
$$

We are now ready to prove

Lemma 18.3 Let $L_{i}^{(v)}, \boldsymbol{c}=\left(c_{i v}\right)(v \in \mathfrak{M}(K), i=1, \ldots, n)$ be as in (6.1) - (6.8). Suppose $\varepsilon=\varepsilon(Q)$ is a small positive number and let $\boldsymbol{g}_{1}=\boldsymbol{g}_{1}(Q), \ldots, \boldsymbol{g}_{n}=\boldsymbol{g}_{n}(Q)$ be linearly 
independent points in $\overline{\mathbb{Q}}^{n}$ satisfying (18.1).

Suppose $1 \leq p \leq n-1$, and let $T_{p}=T_{p}(Q)$ be the linear subspace of $\overline{\mathbb{Q}}^{n}$ generated by $\boldsymbol{g}_{1}, \ldots, \boldsymbol{g}_{p}$.

Suppose

$$
0<\delta<1
$$

Let $m_{1}$ be given by

$$
m_{1}=\left[100 \cdot 2^{6 n+8} n^{7} \delta^{-2} \log 2 r\right] .
$$

Then there exist $p$-dimensional subspaces $T_{1}, \ldots, T_{t}$ of $\overline{\mathbb{Q}}^{n}$ with

$$
t \leq 2^{(n+6)^{2}} n^{3(n+4)} \delta^{-n-4} \log 2 r \log \log 2 r
$$

having the following property:

For every $Q$ with

$$
Q^{\delta}>(2 \mathcal{H})^{n 2^{2 n+3} R m_{1}^{3 m_{1}}}
$$

and

$$
\lambda_{p}(Q)<Q^{-\delta / n} \lambda_{p+1}(Q)
$$

the subspace $T_{p}(Q)$ is among $T_{1}, \ldots, T_{t}$.

Proof. We first remark that although the points $\boldsymbol{g}_{1}(Q), \ldots, \boldsymbol{g}_{p}(Q)$ are not uniquely determined, the space $T_{p}(Q)$ in view of (18.47) and by Corollary 7.5 will be unique, provided $\varepsilon$ is small enough.

We start with the points $\boldsymbol{g}_{1}(Q), \ldots, \boldsymbol{g}_{n}(Q)$. We then apply Lemma 9.2, to obtain points $\boldsymbol{h}_{1}(Q), \ldots, \boldsymbol{h}_{n}(Q)$ satisfying (9.18) and (9.19). In particular these points will satisfy

$$
\operatorname{span}\left\{\boldsymbol{h}_{1}(Q), \ldots, \boldsymbol{h}_{p}(Q)\right\}=\operatorname{span}\left\{\boldsymbol{g}_{1}(Q), \ldots, \boldsymbol{g}_{p}(Q)\right\}=T_{p}(Q) .
$$

We then apply Lemma 10.1 with

$$
k=n-p
$$

The conclusion is that for each $Q$ under consideration there is a permutation $\pi=\pi(Q)$ such that the points $\boldsymbol{h}_{\tau_{1}}, \ldots, \boldsymbol{h}_{\tau_{N}}$ constructed from $\boldsymbol{h}_{1}, \ldots, \boldsymbol{h}_{n}$ satisfy (18.7) with (18.4). We now put

$$
B=\left[3 n^{2} 2^{n} \delta^{-1}\right]
$$


and we apply Lemma 18.1.

Notice that by (18.44) and (18.46) we have (18.8). Hence by Lemma 18.1 the set of $Q$ with (18.46) may be covered by

$$
G \leq(2 n B)^{n} \leq\left(6 n^{3} 2^{n} \delta^{-1}\right)^{n}
$$

subsets $\mathcal{A}_{1}, \ldots, \mathcal{A}_{G}$.

From now on we fix such a subset $\mathcal{A}_{g}$ and for simplicity we call it $\mathcal{A}$. Then for $Q \in \mathcal{A}$ we have by Lemma 18.1 assertions (18.10), (18.11), (18.12).

We then see that the points $\boldsymbol{h}_{\tau_{1}}, \ldots, \boldsymbol{h}_{\tau_{N-1}}$ also satisfy (18.28) of Lemma 18.2.

Defining the tuple $\left(f_{\tau_{i} v}\right)(v \in \mathfrak{M}(K), i=1, \ldots, N)$ as in (18.31), we finally may conclude with (18.42) that for $Q \in \mathcal{A}$ the parallelepiped $\Pi^{(k)}\left(Q^{2 n^{2}}, \boldsymbol{f}\right)$ has penultimate minimum $\nu_{N-1}\left(Q^{2 n^{2}}\right)$ satisfying

$$
\nu_{N-1}\left(Q^{2 n^{2}}\right) \leq(1+\varepsilon) Q^{\frac{n}{B}}\left(\lambda_{\tau_{N-1}}(Q) / \lambda_{\tau_{N}}(Q)\right)^{1 / N} .
$$

With $k=n-p$ as in (18.50) we have $\tau_{N-1}=\{p, p+2, \ldots, n\}, \tau_{N}=\{p+1, p+2, \ldots, n\}$. Therefore $\lambda_{N-1} / \lambda_{N}=\left(\lambda_{p} \lambda_{p+2} \ldots \lambda_{n}\right) /\left(\lambda_{p+1} \lambda_{p+2} \ldots \lambda_{n}\right)=\lambda_{p} / \lambda_{p+1}$.

Thus by (18.47)

$$
\lambda_{N-1} / \lambda_{N}<Q^{-\delta / n}
$$

Combining this with (18.50), (18.52) we get

$$
\nu_{N-1}\left(Q^{2 n^{2}}\right) \leq(1+\varepsilon) Q^{\frac{n}{B}-\delta / n N}<Q^{-\frac{\delta}{n \cdot 2^{n+1}}}
$$

provided $\varepsilon$ is small enough.

We want to apply Lemma 16.2 with the points $\gamma \boldsymbol{h}_{\tau_{1}}(Q), \ldots, \gamma \boldsymbol{h}_{\tau_{N-1}}(Q)$ instead of $\boldsymbol{g}_{1}(Q), \ldots, \boldsymbol{g}_{n-1}(Q)$ and with $\Pi^{(k)}\left(Q^{2 n^{2}}, \boldsymbol{f}\right)$ instead of $\Pi(Q, \boldsymbol{c})$. By (18.53) the analogue of (16.23), with $Q^{2 n^{2}}$ in place of $Q$, is satisfied with $\delta$ replaced by

$$
\delta_{1}=\frac{\delta}{n^{3} 2^{n+2}} .
$$

On the other hand in the definition of $\Pi^{(k)}\left(Q^{2 n^{2}}, \boldsymbol{f}\right)$, by (18.40) the parameters $r$ and $n$ are replaced by $\left(\begin{array}{l}r \\ k\end{array}\right)$ and $\left(\begin{array}{l}n \\ k\end{array}\right)$ respectively.

Writing $m_{1}$ for the parameter which corresponds to $m$ in (16.20), we now have to require

$$
m_{1}>1600\left(\begin{array}{l}
n \\
k
\end{array}\right)^{4} \delta_{1}^{-2} \log \left(2\left(\begin{array}{l}
r \\
k
\end{array}\right)\right) .
$$

Similarly $E$ in (16.21) has to be replaced by

$$
E_{1}=41 m_{1}^{2}\left(\begin{array}{l}
n \\
k
\end{array}\right)^{2} \delta_{1}^{-1}=41 m_{1}^{2}\left(\begin{array}{l}
n \\
p
\end{array}\right)^{2} \delta_{1}^{-1} .
$$

With $\delta_{1}$ from (18.54) we may choose

$$
m_{1}=\left[100 \cdot 2^{6 n+8} n^{7} \delta^{-2} \log 2 r\right] .
$$


This is the value in (18.44).

We still have to guarantee the analogue of (16.24). As seen in the discussion preceding Lemma 18.3 the parameter $R$ remains unchanged and therefore we have

$$
R \leq\left(\begin{array}{l}
r \\
n
\end{array}\right) .
$$

On the other hand, in view of (18.36), $\mathcal{H}$ has to be replaced by

$$
\mathcal{H}^{\frac{k}{n}\left(\begin{array}{l}
n \\
k
\end{array}\right)} \text {. }
$$

Thus the analogue of (16.24) is satisfied provided

$$
\left(Q^{2 n^{2}}\right)^{\delta_{1}}>\left(2 \mathcal{H}^{\frac{k}{n}\left(\begin{array}{l}
n \\
k
\end{array}\right)}\right)^{3 R m_{1}^{3 m_{1}}}
$$

With $\delta_{1}$ from (18.54) we obtain (18.59) as an easy consequence of (18.46).

So, all hypotheses of Lemma 16.2 are satisfied.

The conclusion is as follows:

For $Q$ in $\mathcal{A}$ satisfying (18.46) and (18.47) let $T^{(k)}(Q)$ be the subspace of $\overline{\mathbb{Q}}^{N}$ generated by $\boldsymbol{h}_{\tau_{1}}(Q), \ldots, \boldsymbol{h}_{\tau_{N-1}}(Q)$. Then there exist proper linear subspaces $T_{1}^{(k)}, \ldots, T_{a}^{(k)}$ of $\overline{\mathbb{Q}}^{\left(\begin{array}{l}n \\ k\end{array}\right)}$ with

$$
a<m_{1}\left(1+\frac{5000}{999} \delta_{1}^{-1} \log E_{1}\right)
$$

such that for each $Q$ under consideration $T^{(k)}(Q)$ coincides with one of $T_{1}^{(k)}, \ldots T_{a}^{(k)}$.

We now apply Lemma 10.3. Consequently there is a collection of $p$-dimensional subspaces $T_{1}, \ldots, T_{a}$ of $\overline{\mathbb{Q}}^{n}$ such that for each $Q$ under consideration the subspace $T_{p}(Q)$ generated by $\boldsymbol{g}_{1}(Q), \ldots, \boldsymbol{g}_{p}(Q)$ coincides with one of $T_{1}, \ldots, T_{a}$.

Introducing a factor $\left(6 n^{3} 2^{n} \delta^{-1}\right)^{n}$ for the number of possible sets $\mathcal{A}$ from (18.51), we finally see that

$$
t<\left(6 n^{3} 2^{n} \delta^{-1}\right)^{n} m_{1}\left(1+\frac{5000}{999} \delta_{1}^{-1} \log E_{1}\right)
$$

subspaces $T_{1}, \ldots, T_{t}$ will suffice.

Using (18.54), (18.55), (18.56) we get

$$
\begin{aligned}
& \log E_{1}<\log 41+2 \log m_{1}+2 n \log 2+3 \log n+(n+2) \log 2+\log \delta^{-1} \\
& \log m_{1} \leq \log 100+(6 n+8) \log 2+7 \log n+2 \log \delta^{-1}+\log \log 2 r .
\end{aligned}
$$

Since we may assume that $r>1$, we have $4 \log \log 2 r>1$. Therefore

$$
\log E_{1}<6 \log \log 2 r\left(\log (410000)+(15 n+18) \log 2+17 \log n+5 \delta^{-1}\right) .
$$

Altogether we see that the right hand side in (18.61) does not exceed

$$
2^{(n+6)^{2}} n^{3(n+4)} \delta^{-n-4} \log 2 r \log \log 2 r .
$$

This is the bound in (18.45) and Lemma 18.3 follows. 


\section{Proof of Proposition 6.1, and hence of Theorem 2.1}

With the notation from (6.1) - (6.8), (6.10) - (6.15) we have to deal with the points $\boldsymbol{x} \in \overline{\mathbb{Q}}^{n}$ lying in

$$
Q^{-\delta} * \Pi(Q, \boldsymbol{c})
$$

for parameters $Q$ satisfying

$$
Q>\max \left\{\mathcal{H}^{1 /\left(\begin{array}{l}
r \\
n
\end{array}\right)}, n^{1 / \delta}\right\}
$$

We distinguish large and small parameters $Q$. We call $Q$ small if

$$
\max \left\{\mathcal{H}^{1 /\left(\begin{array}{l}
r \\
n
\end{array}\right)}, n^{1 / \delta}\right\}<Q \leq\left(\max \left\{\mathcal{H}^{1 /\left(\begin{array}{l}
r \\
n
\end{array}\right)}, n^{1 / \delta}\right\}\right)^{\delta^{-1} n 2^{2 n+4}\left(\begin{array}{c}
r \\
n
\end{array}\right)^{2} m_{1}^{3 m_{1}}}
$$

where $m_{1}$ is as in (18.44), i.e.,

$$
m_{1}=\left[100 \cdot 2^{6 n+8} n^{7} \delta^{-2} \log 2 r\right] .
$$

Parameters with $Q$ with

$$
Q>\left(\max \left\{\mathcal{H}^{1 /\left(\begin{array}{l}
r \\
n
\end{array}\right)}, n^{1 / \delta}\right\}\right)^{\delta^{-1} n 2^{2 n+4}\left(\begin{array}{l}
r \\
n
\end{array}\right)^{2} m_{1}^{3 m_{1}}}
$$

are called large.

To treat the small values of $Q$ we apply Lemma 8.1. To cover the range (19.3), by (8.2) we do not need more than

$$
\begin{aligned}
& 1+5 \delta^{-1}\left(\log \delta^{-1}+\log n+(2 n+4) \log 2+2 \log \left(\begin{array}{l}
r \\
n
\end{array}\right)+3 m_{1} \log m_{1}\right) \\
& <31 \delta^{-1} m_{1} \log m_{1}
\end{aligned}
$$

subspaces.

We now treat the values $Q$ satisfying (19.5). For these $Q$ we want to apply Lemma 18.3. We first notice that the parameter $R$ in (18.46) satisfies $R \leq\left(\begin{array}{l}r \\ n\end{array}\right)$. Moreover, since $0<\delta<1$ and $n \geq 2$, we have $\max \left\{\mathcal{H}^{1 /\left(\begin{array}{l}r \\ n\end{array}\right)}, n^{1 / \delta}\right\} \geq(2 \mathcal{H})^{1 /\left(2\left(\begin{array}{c}r \\ n\end{array}\right)\right)}$. Thus (19.5) implies (18.46).

We next show that we have only to consider such values $Q$ for which (18.47) is satisfied for a suitable $p$ with $1 \leq p \leq n-1$.

If $Q$ is such that there exists $\boldsymbol{x} \in \overline{\mathbb{Q}}^{n}, \boldsymbol{x} \neq 0$ with

$$
\boldsymbol{x} \in Q^{-\delta} * \Pi(Q, \boldsymbol{c}),
$$


then

$$
\lambda_{1}(Q) \leq Q^{-\delta}
$$

On the other hand by Corollary 7.6

$$
\lambda_{1}(Q) \ldots \lambda_{n}(Q) \geq n^{-\frac{n}{2}}
$$

and so by (19.5), (19.8)

$$
\lambda_{n}(Q) \geq n^{-\frac{n}{2(n-1)}} Q^{\delta /(n-1)}>Q^{\delta / n} .
$$

Comparing (19.8) and (19.9) we may conclude that there exists $q$ with $1 \leq q \leq n-1$ such that

$$
\lambda_{q}(Q)<Q^{-\delta / n} \lambda_{q+1}(Q)
$$

Let $p=p(Q)$ be largest with this property. Then by (19.9) and (19.10) we have

$$
\lambda_{p+1}(Q)>Q^{\delta / n-(n-p-1) \delta / n}=Q^{(-n+p+2) \delta / n}>Q^{-\delta} .
$$

Now let $\boldsymbol{g}_{1}(Q), \ldots, \boldsymbol{g}_{p}(Q), \boldsymbol{g}_{p+1}(Q), \ldots, \boldsymbol{g}_{n}(Q)$ be linearly independent points in $\overline{\mathbb{Q}}^{n}$ with

$$
\boldsymbol{g}_{i}(Q) \in(1+\varepsilon) \lambda_{i}(Q) * \Pi(Q, \boldsymbol{c}),
$$

where $\varepsilon=\varepsilon(Q)$ is small enough such that $1+\varepsilon<Q^{\delta / n}$. The definition of successive minima implies that then any point $\boldsymbol{x}$ with (19.7) lies in the $p$-dimensional subspace $T_{p}(Q)$ generated by $\boldsymbol{g}_{1}(Q), \ldots, \boldsymbol{g}_{p}(Q)$. We partition the set of $Q$ satisfying (19.5) into $n-1$ subsets corresponding to the $n-1$ possibilities for $p$.

For each $p$, by (19.10) and (19.5) we may apply Lemma 18.3. So for each $p$, by (18.45)

$$
t<2^{(n+6)^{2}} n^{3(n+4)} \delta^{-n-4} \log 2 r \log \log 2 r
$$

subspaces will suffice.

The bound (19.11), i.e., (18.45) exceeds the bound (18.61), which in turn exceeds the bound (19.6) for small parameters. Introducing in (19.11) a factor $n$ for the $n-1$ possibilities of $p$ and to take care of the small values of $Q$, we finitely see that altogether

$$
\begin{aligned}
t_{4}(n, r, \delta) & \leq n 2^{(n+6)^{2}} n^{3(n+4)} \delta^{-n-4} \log (2 r) \log \log (2 r) \\
& \leq 4^{(n+6)^{2}} \delta^{-n-4} \log (2 r) \log \log (2 r)
\end{aligned}
$$

subspaces suffice, and this is the assertion of Proposition 6.1. 


\section{Simultaneous Inequalities}

In order to prove Theorem 3.1 we will derive from Theorem 2.1 an auxiliary result which however may be of some independent interest and which we therefore formulate as a theorem. As in section 3 we start with a number field $E$ and a finite subset $S$ of $\mathfrak{M}(E)$ of cardinality $s$. For $v \in S$ we write \|\|$_{v}$ for the normalized absolute value corresponding to $v$ (cf. (1.7), (1.8)) and we suppose that \|\|$_{v}$ is extended to $\overline{\mathbb{Q}}$ as described in (3.7).

Let $\left\{L_{1}, \ldots, L_{r}\right\}$ be a family of linear forms in $\boldsymbol{X}=\left(X_{1}, \ldots, X_{n}\right)$ with coefficients in $\overline{\mathbb{Q}}$ satisfying

$$
\operatorname{rank}\left(L_{1}, \ldots, L_{r}\right)=n
$$

and

$$
L_{i} \text { has some coefficient equal to } 1 \quad(i=1, \ldots, r) \text {. }
$$

Suppose that for each $v \in S$ we are given a set $\left\{L_{1}^{(v)}, \ldots, L_{n}^{(v)}\right\}$ of forms with

$$
\left\{L_{1}^{(v)}, \ldots, L_{n}^{(v)}\right\} \subset\left\{L_{1}, \ldots, L_{r}\right\}, \quad \operatorname{rank}\left\{L_{1}^{(v)}, \ldots, L_{n}^{(v)}\right\}=n
$$

Write

$$
\Delta_{v}=\left\|\operatorname{det}\left(L_{1}^{(v)}, \ldots, L_{n}^{(v)}\right)\right\|_{v}
$$

For $(i, v)(v \in S, i=1, \ldots, n)$ let $E\left(L_{i}^{(v)}\right)$ be the extension of $E$ generated by the coefficients of $L_{i}^{(v)}$ and let $D$ be a natural number satisfying

$$
\left[E\left(L_{i}^{(v)}\right): E\right] \leq D \quad(v \in S, i=1, \ldots, n) .
$$

Suppose moreover that $H$ is a quantity with

$$
H\left(L_{i}^{(v)}\right) \leq H \quad(v \in S, i=1, \ldots, n) .
$$

We prove

Theorem 20.1 Assume (20.1) - (20.6). Suppose $0<\delta \leq 1$ and let $\boldsymbol{e}=\left(e_{i v}\right)(v \in S$, $i=1, \ldots, n)$ be a tuple of real numbers satisfying

$$
\begin{gathered}
\sum_{v \in S} \sum_{i=1}^{n} e_{i v} \leq-n-\delta, \\
\sum_{v \in S} \max \left\{e_{1 v}, \ldots, e_{n v}\right\} \leq n .
\end{gathered}
$$


Then there are proper linear subspaces $T_{1}, \ldots, T_{t_{5}}$ of $\overline{\mathbb{Q}}^{n}$, all defined over $E$, where

$$
t_{5}=t_{5}(n, r, D, \delta) \leq 2^{3(n+8)^{2}} \delta^{-n-4} \log (4 r D) \log \log (4 r D)
$$

with the following property:

The set of solutions $\boldsymbol{x} \in \overline{\mathbb{Q}}^{n}$ of the simultaneous inequalities

$$
\max _{\sigma \in \operatorname{Gal}(\overline{\mathbb{Q}} / E)} \frac{\left\|L_{i}^{(v)}(\sigma(\boldsymbol{x}))\right\|_{v}}{\Delta_{v}^{1 / n}\|\sigma(\boldsymbol{x})\|_{v}} \leq H(\boldsymbol{x})^{e_{i v}} \quad(v \in S, i=1, \ldots, n)
$$

$$
H(\boldsymbol{x})>\max \left\{H, n^{2 n / \delta}\right\}
$$

is contained in the union

$$
T_{1} \cup \ldots \cup T_{t_{5}}
$$

We emphasize that the bound in (20.9) for the number of subspaces does not depend upon the field $E$, nor does it depend upon the set $S$.

To prove Theorem 20.1 we will construct a twisted height $H_{Q, c}(\boldsymbol{x})$ such that for any solution $\boldsymbol{x}$ of (20.10) the quantity $\max _{\sigma \in \operatorname{Gal}(\overline{\mathbb{Q}} / E)} H_{Q, \boldsymbol{c}}(\sigma(\boldsymbol{x}))$ will be small. We will then apply Corollary 2.2 and thereby deduce the assertion.

We begin with the construction of the height $H_{Q, \boldsymbol{c}}(\boldsymbol{x})$. For a linear form $L=\alpha_{1} X_{1}+\ldots+\alpha_{n} X_{n}$ with coefficients $\alpha_{1}, \ldots, \alpha_{n} \in \overline{\mathbb{Q}}$ and for an automorphism $\sigma \in \operatorname{Gal}(\overline{\mathbb{Q}} / E)$ we write $\sigma L=\sigma\left(\alpha_{1}\right) X_{1}+\ldots+\sigma\left(\alpha_{n}\right) X_{n}$. We fix a finite normal extension $F$ of $E$ containing the coefficients of the forms $\sigma L_{i}(\sigma \in \operatorname{Gal}(\overline{\mathbb{Q}} / E), i=1, \ldots, r)$. Write $T$ for the set of places $w \in \mathfrak{M}(F)$ lying above the places $v \in S$. For each place $w \in T, w \mid v, v \in S$, there is an automorphism $\sigma_{w} \in \operatorname{Gal}(F / E)$ such that

$$
\|x\|_{w}=\left\|\sigma_{w}(x)\right\|_{v}^{d(w / v)} \quad \text { for each } x \in F .
$$

For each $w \in T$ we fix such a $\sigma_{w}$ and we define the forms $L_{1}^{*(w)}, \ldots, L_{n}^{*(w)}$ by

$$
L_{i}^{*(w)}=\sigma_{w}^{-1} L_{i}^{(v)} .
$$

Let $\mathcal{F}$ be the family of linear forms

$$
\left\{\sigma L \mid \sigma \in \operatorname{Gal}(\overline{\mathbb{Q}} / E), \quad L \in\left\{L_{1}, \ldots, L_{r}, X_{1}, \ldots, X_{n}\right\}\right\} .
$$

By $(20.5) \mathcal{F}$ has cardinality

$$
\operatorname{card} \mathcal{F} \leq r D+n \leq 2 r D
$$

Clearly all the forms (20.13) lie in $\mathcal{F}$. By repeating some of the forms in $\mathcal{F}$, if necessary, we get a family of forms $\mathcal{F}_{1}$ containing $\mathcal{F}$ such that

$$
\operatorname{card} \mathcal{F}_{1}=2 r D
$$


Now let $F^{\prime}$ be a finite extension of $F$ such that moreover $F^{\prime} / E$ is normal. Write $T^{\prime}$ for the set of places $w^{\prime} \in \mathfrak{M}\left(F^{\prime}\right)$ lying above the places in $S$ (and hence also above the places in $T$ ). If $w^{\prime} \in T^{\prime}$ lies above $w \in T$ and $w$ in turn lies above $v \in S$ we can find an element $\sigma_{w^{\prime}} \in \operatorname{Gal}\left(F^{\prime} / E\right)$ with $\sigma_{w^{\prime} / F}=\sigma_{w}$ such that for each $x \in F^{\prime}$

$$
\|x\|_{w^{\prime}}=\left\|\sigma_{w^{\prime}}(x)\right\|_{v}^{d\left(w^{\prime} / v\right)}
$$

Moreover putting $L_{i}^{*\left(w^{\prime}\right)}=L_{i}^{*(w)}$, we get $L_{i}^{*\left(w^{\prime}\right)}=\sigma_{w}^{-1} L_{i}^{(v)}=\sigma_{w^{\prime}}^{-1} L_{i}^{(v)}$. In other words, relations (20.12), (20.13) remain valid with $F, T, w$ replaced by $F^{\prime}, T^{\prime}, w^{\prime}$ respectively, independently of the particular choice of $\sigma_{w^{\prime}}$. For $F^{\prime}$ and $w^{\prime} \in T^{\prime}$ as above we put

$$
\Delta_{w^{\prime}}^{*}=\left\|\operatorname{det}\left(L_{1}^{*\left(w^{\prime}\right)}, \ldots, L_{n}^{*\left(w^{\prime}\right)}\right)\right\|_{w^{\prime}} .
$$

Lemma 20.2 Let $\boldsymbol{x} \in \overline{\mathbb{Q}}^{n}$ be a solution of (20.10). Let $F^{\prime}$ be a finite extension of $F$ such that $F^{\prime} / E$ is normal and such that $\boldsymbol{x} \in F^{\prime n}$. Then we have

$$
\max _{\sigma \in \operatorname{Gal}(\mathbb{\mathbb { Q }} / E)} \frac{\left\|L_{i}^{*\left(w^{\prime}\right)}(\sigma(\boldsymbol{x}))\right\|_{w^{\prime}}}{\Delta_{w^{\prime}}^{* 1 / n}\|\sigma(\boldsymbol{x})\|_{w^{\prime}}} \leq H(\boldsymbol{x})^{e_{i v} d\left(w^{\prime} / v\right)} \quad\left(w^{\prime} \in T^{\prime}, i=1, \ldots, n\right) .
$$

In (20.16) $v \in S$ is the place lying below $w^{\prime}$.

Remark We are concentrating on normal extensions $F^{\prime}$ of $E$ to guarantee that for each $\sigma \in G a l(\overline{\mathbb{Q}} / E)$ the point $\sigma(\boldsymbol{x})$ lies in $F^{\prime}$.

Proof. Using (20.12), (20.13) we get

$$
\begin{aligned}
& \frac{\left\|L_{i}^{*\left(w^{\prime}\right)}(\sigma(\boldsymbol{x}))\right\|_{w^{\prime}}}{\|\sigma(\boldsymbol{x})\|_{w^{\prime}}}=\frac{\left\|\left(\sigma_{w^{\prime}}^{-1} L_{i}^{(v)}\right)(\sigma(\boldsymbol{x}))\right\|_{w^{\prime}}}{\|\sigma(\boldsymbol{x})\|_{w^{\prime}}} \\
& \quad=\frac{\| \sigma_{w^{\prime}}\left(\left(\sigma_{w^{\prime}}^{-1} L_{i}^{(v)}\right)(\sigma(\boldsymbol{x})) \|_{v}^{d\left(w^{\prime} / v\right)}\right.}{\left\|\sigma_{w^{\prime}}(\sigma(\boldsymbol{x}))\right\|_{v}^{d\left(w^{\prime} / v\right)}}=\frac{\left\|L_{i}^{(v)}\left(\sigma_{w^{\prime}} \sigma(\boldsymbol{x})\right)\right\|_{v}^{d\left(w^{\prime} / v\right)}}{\left\|\sigma_{w^{\prime}} \sigma(\boldsymbol{x})\right\|_{v}^{d\left(w^{\prime} / v\right)}} .
\end{aligned}
$$

Moreover, we infer from (20.15) that

$$
\begin{aligned}
\Delta_{w^{\prime}}^{*} & =\left\|\operatorname{det}\left(L_{1}^{*\left(w^{\prime}\right)}, \ldots, L_{n}^{*\left(w^{\prime}\right)}\right)\right\|_{w^{\prime}}=\| \operatorname{det}\left(\sigma_{w^{\prime}}^{-1} L_{1}^{(v)}, \ldots, \sigma_{w^{\prime}}^{-1} L_{n}^{(v)} \|_{w^{\prime}}\right. \\
& =\left\|\sigma_{w^{\prime}}\left(\operatorname{det}\left(\sigma_{w^{\prime}}^{-1} L_{1}^{(v)}, \ldots, \sigma_{w^{\prime}}^{-1} L_{n}^{(v)}\right)\right)\right\|_{v}^{d\left(w^{\prime} / v\right)}=\left\|\operatorname{det}\left(L_{1}^{(v)}, \ldots, L_{n}^{(v)}\right)\right\|_{v}^{d\left(w^{\prime} / v\right)} \\
& =\Delta_{v}^{d\left(w^{\prime} / v\right)} .
\end{aligned}
$$

Thus for $\sigma \in \operatorname{Gal}(\overline{\mathbb{Q}} / E)$ we obtain

$$
\frac{\left\|L_{i}^{*\left(w^{\prime}\right)}(\sigma(\boldsymbol{x}))\right\|_{w^{\prime}}}{\Delta_{w^{\prime}}^{* 1 / n}\|\sigma(\boldsymbol{x})\|_{w^{\prime}}}=\left(\frac{\left\|L_{i}^{(v)}\left(\sigma_{w^{\prime}} \sigma(\boldsymbol{x})\right)\right\|_{v}}{\Delta_{v}^{1 / n}\left\|\sigma_{w^{\prime}} \sigma(\boldsymbol{x})\right\|_{v}}\right)^{d\left(w^{\prime} / v\right)} .
$$

The assertion now follows from (20.10). 
Lemma 20.2 enables us to define the desired twisted height.

Let $F$ and $T \subset \mathfrak{M}(F)$ be as above. For $w \in \mathfrak{M}(F)$ and for $i=1, \ldots, n$ we define the linear forms $M_{i}^{(w)}$ by

$$
M_{i}^{(w)}= \begin{cases}L_{i}^{*(w)} & \text { for } w \in T \\ X_{i} & \text { for } w \notin T .\end{cases}
$$

By (20.3) and (20.13) we have

$$
\operatorname{rank}\left\{M_{1}^{(w)}, \ldots, M_{n}^{(w)}\right\}=n \quad \text { for each } w \in \mathfrak{M}(F) .
$$

Moreover in view of our construction and in view of (20.3), each form $M_{i}^{(w)}$ belongs to the family $\mathcal{F}_{1}$ in $(20.14)$.

In (20.7), (20.8) we may suppose without loss of generality that

$$
\sum_{v \in S} \sum_{i=1}^{n} e_{i v}=-n-\delta \quad \text { and } \quad \sum_{v \in S} \max \left\{e_{1 v}, \ldots, e_{n v}\right\} \leq n .
$$

For assume that initially we do not have (20.18). For $v \in S$ let $i(v)$ be an index with

$$
e_{i(v), v}=\max \left\{e_{1 v}, \ldots, e_{n v}\right\} .
$$

We may choose elements $e_{i(v), v}^{\prime} \geq e_{i v}$ such that $\sum_{v \in S} e_{i(v), v}^{\prime}$ is maximal with

$$
\sum_{v \in S} e_{i(v), v}^{\prime} \leq n \quad \text { and } \quad \sum_{v \in S} e_{i(v), v}^{\prime}+\sum_{v \in S} \sum_{i \neq i(v)} e_{i v} \leq-n-\delta
$$

Now either $\sum_{v \in S} e_{i(v), v}^{\prime}+\sum_{v \in S} \sum_{i \neq i(v)} e_{i v}=-n-\delta$.

Then in Theorem 20.1 we may replace the elements $e_{i(v), v}$ by $e_{i(v), v}^{\prime}$ and the analogue of (20.18) will be true.

Or we have $\sum_{v \in S} e_{i(v), v}^{\prime}+\sum_{v \in S} \sum_{i \neq i(v)} e_{i v}<-n-\delta$ and $\sum_{v \in S} e_{i(v), v}^{\prime}=n$. Then clearly we can find for $i \neq i(v)$ elements $e_{i v}^{\prime}$ with $e_{i v} \leq e_{i v}^{\prime} \leq e_{i(v), v}^{\prime}$ such that $\sum_{v \in S} \sum_{i=1}^{n} e_{i v}^{\prime}=-n-\delta$. Again with $e_{i v}$ replaced by $e_{i v}^{\prime}$ the analogue of (20.18) will be true. Moreover the analogue of (20.10) with $e_{i v}$ replaced by $e_{i v}^{\prime}$ obviously will be satisfied as well. We define the tuple $\boldsymbol{c}=\left(c_{i w}\right)(w \in \mathfrak{M}(F), i=1, \ldots, n)$ by

$$
c_{i w}= \begin{cases}d(w / v)\left(e_{i v}+\frac{1}{s}(1+\delta / n)\right) & (w \in T, w \mid v, v \in S, i=1, \ldots n) \\ 0 & (w \in \mathfrak{M}(F) \backslash T, i=1, \ldots, n) .\end{cases}
$$

Combination of (20.18) and (20.19) yields

$$
\sum_{w \in \mathfrak{M}(F)} \sum_{i=1}^{n} c_{i w}=\sum_{v \in S} \sum_{i=1}^{n}\left(e_{i v}+\frac{1}{s}(1+\delta / n)\right)=-n-\delta+\frac{n s}{s}(1+\delta / n)=0 .
$$


Moreover we get

$$
\begin{aligned}
\sum_{w \in \mathfrak{M}(F)} \max \left\{c_{1 w}, \ldots, c_{n w}\right\} & =\sum_{v \in S}\left(\max \left\{e_{1 v}, \ldots, e_{n v}\right\}+\frac{1}{s}(1+\delta / n)\right) \\
& \leq n+1+\delta / n \leq n+2
\end{aligned}
$$

For a finite extension $F^{\prime}$ of $F$ and for $w^{\prime} \in \mathfrak{M}\left(F^{\prime}\right)$ lying above $w \in \mathfrak{M}(F)$ we define for $i=1, \ldots, n$

$$
M_{i}^{\left(w^{\prime}\right)}=M_{i}^{(w)}, \quad c_{i w^{\prime}}=d\left(w^{\prime} / w\right) c_{i w}
$$

and we write

$$
\Delta_{w^{\prime}}=\left\|\operatorname{det}\left(M_{1}^{\left(w^{\prime}\right)}, \ldots, M_{n}^{\left(w^{\prime}\right)}\right)\right\|_{w^{\prime}} .
$$

Finally, for $\boldsymbol{x} \in \overline{\mathbb{Q}}^{n}$ we define the height $H_{Q, c}(\boldsymbol{x})$ as follows. If $F^{\prime}$ is a finite extension of $F$ such that $\boldsymbol{x} \in F^{\prime n}$ then we put

$$
H_{Q, \boldsymbol{c}}(\boldsymbol{x})=\prod_{w^{\prime} \in \mathfrak{M}\left(F^{\prime}\right)} \max _{1 \leq i \leq n} \frac{\left\|M_{i}^{\left(w^{\prime}\right)}(\boldsymbol{x})\right\|_{w^{\prime}}}{\Delta_{w^{\prime}}^{1 / n} Q^{c_{i w^{\prime}}}} .
$$

Lemma 20.3 Let $\boldsymbol{x} \in \overline{\mathbb{Q}}^{n} \backslash\{\mathbf{0}\}$ be a solution of (20.10). Then, writing

$$
Q=H(\boldsymbol{x})
$$

we have

$$
\max _{\sigma \in \operatorname{Gal}(\overline{\mathbb{Q}} / E)} H_{Q, c}(\sigma(\boldsymbol{x})) \leq Q^{-\delta / n}
$$

Proof. We choose a finite extension $F^{\prime}$ of $F$ such that $\boldsymbol{x} \in F^{\prime n}$ and such that $F^{\prime} / E$ is normal. Then by Lemma 20.2, $\boldsymbol{x}$ satisfies (20.16). Combination of (20.16), (20.17), (20.19) and (20.23) implies for $w^{\prime} \in T^{\prime}$ and for $\sigma \in \operatorname{Gal}(\overline{\mathbb{Q}} / E)$

$$
\begin{aligned}
\frac{\left\|M_{i}^{\left(w^{\prime}\right)}(\sigma(\boldsymbol{x}))\right\|_{w^{\prime}}}{\Delta_{w^{\prime}}^{1 / n} Q^{c_{i w^{\prime}}}}= & \frac{\left\|M_{i}^{\left(w^{\prime}\right)}(\sigma(\boldsymbol{x}))\right\|_{w^{\prime}}}{\Delta_{w^{\prime}}^{1 / n} H(\boldsymbol{x})^{d\left(w^{\prime} / v\right)\left(e_{i v}+\frac{1}{s}(1+\delta / n)\right)}} \\
\leq & \|\sigma(\boldsymbol{x})\|_{w^{\prime}} H(\boldsymbol{x})^{\left.-d\left(w^{\prime} / v\right) \frac{1}{s}(1+\delta / n)\right)} \\
& \left(w^{\prime} \mid v, v \in S ; i=1, \ldots, n\right) .
\end{aligned}
$$

Moreover, for $w^{\prime} \in \mathfrak{M}\left(F^{\prime}\right) \backslash T^{\prime}$ we have $\Delta_{w^{\prime}}=1$ and so by (20.17) and (20.19) we get for $i=1, \ldots, n$

$$
\frac{\left\|M_{i}^{\left(w^{\prime}\right)}(\sigma(\boldsymbol{x}))\right\|_{w^{\prime}}}{\Delta_{w^{\prime}}^{1 / n} Q^{c_{i w^{\prime}}}}=\left\|\sigma\left(x_{i}\right)\right\| w^{\prime} \leq\|\sigma(\boldsymbol{x})\|_{w^{\prime}} .
$$


In view of (20.25), (20.26) we obtain

$$
\begin{aligned}
H_{Q, \boldsymbol{c}}(\sigma(\boldsymbol{x})) & =\prod_{w^{\prime} \in \mathfrak{M}\left(F^{\prime}\right)} \max _{1 \leq i \leq n} \frac{\left\|M_{i}^{\left(w^{\prime}\right)}(\sigma(\boldsymbol{x}))\right\|_{w^{\prime}}}{\Delta_{w^{\prime}}^{1 / n}\|\sigma(\boldsymbol{x})\|_{w^{\prime}}} \\
& \leq\left(\prod_{w^{\prime} \in \mathfrak{M}\left(F^{\prime}\right)}\|\sigma(\boldsymbol{x})\|_{w^{\prime}}\right) \prod_{w^{\prime} \in T^{\prime}} H(\boldsymbol{x})^{-d\left(w^{\prime} / v\right) \frac{1}{s}(1+\delta / n)} .
\end{aligned}
$$

However

$$
\prod_{w^{\prime} \in \mathfrak{M}\left(F^{\prime}\right)}\|\sigma(\boldsymbol{x})\|_{w^{\prime}}=H(\sigma(\boldsymbol{x}))=H(\boldsymbol{x}) .
$$

On the other hand

$$
\sum_{w^{\prime} \in T^{\prime}} d\left(w^{\prime} / v\right) \frac{1}{s}(1+\delta / n)=\frac{1}{s}(1+\delta / n) \sum_{w^{\prime} \in T^{\prime}} d\left(w^{\prime} / v\right)=\frac{1}{s}(1+\delta / n) \cdot s=1+\delta / n .
$$

Therefore by (20.23)

$$
H_{Q, \boldsymbol{c}}(\sigma(\boldsymbol{x})) \leq H(\boldsymbol{x})^{-\delta / n}=Q^{-\delta / n}
$$

The Lemma follows.

The proof of Theorem 20.1 now is easily finished. We apply Corollary 2.2 to inequality (20.24). To do so we still have to ensure that its hypotheses are satisfied.

The rôle of the field $K$ containing the coefficients of the forms in Corollary 2.2 now is played by the field $F$. The field $E$ in Corollary 2.2 also in our application will be $E$.

In Corollary 2.2 we have hypothesis (2.14), i.e., $\sum_{v \in \mathfrak{M}(K)} \max \left\{c_{1 v}, \ldots, c_{n v}\right\} \leq 1$.

Instead of this we now have (20.21), i.e.,

$$
\sum_{w \in \mathfrak{M}(F)} \max \left\{c_{1 w}, \ldots, c_{n w}\right\} \leq n+2 .
$$

Write

$$
c_{i w}^{\prime}=\frac{1}{n+2} c_{i w} \quad(w \in \mathfrak{M}(F), i=1, \ldots, n)
$$

and

$$
Q_{1}=Q^{n+2}
$$

We may then define the height $H_{Q_{1}, \boldsymbol{c}^{\prime}}(\boldsymbol{x})$ in an obvious way in analogy with (20.22). For the new tuple $\boldsymbol{c}^{\prime}$ we get

$$
\sum_{w \in \mathfrak{M}(F)} \max \left\{c_{1 w}^{\prime}, \ldots, c_{n w}^{\prime}\right\} \leq 1
$$

i.e., we get the analogue of (2.14). By Lemma 20.3, any solution $\boldsymbol{x} \neq \mathbf{0}$ of (20.10) satisfies

$$
\max _{\sigma \in \operatorname{Gal}(\overline{\mathbb{Q}} / E)} H_{Q_{1}, c^{\prime}}(\sigma(\boldsymbol{x})) \leq Q_{1}^{-\frac{\delta}{n(n+2)}} .
$$


So in the application of Corollary 2.2 we have to replace $\delta$ by $\delta /(n(n+2))$. The rôle played by the family $\left\{L_{1}, \ldots, L_{r}\right\}$ now is played by the family $\mathcal{F}_{1}$ in $(20.14)$. This means in particular that the parameter $r$ in Corollary 2.2 in our current context has to be replaced by $2 r D$. Let $\mathcal{F}_{1}=\left\{M_{1}, \ldots, M_{2 r D}\right\}$. We write $\mathcal{H}=\mathcal{H}\left(M_{1}, \ldots, M_{2 r D}\right)$.

Now Corollary 2.2 implies the following: As $Q_{1}$ runs through values satisfying

$$
Q_{1}>\max \left\{\mathcal{H}^{1 /\left(\begin{array}{c}
2 r D \\
n
\end{array}\right)}, n^{\frac{2 n(n+2)}{\delta}}\right\}
$$

the set of solutions of (20.28) will be contained in the union of not more than

$$
t_{1}(n, 2 r D, \delta /(n(n+2)))
$$

proper linear subspaces of $\overline{\mathbb{Q}}^{n}$. All these subspaces are defined over E. By (2.19)

$$
\begin{aligned}
t_{1}(n, 2 r D, \delta /(n(n+2))) & \leq 4^{(n+8)^{2}}(n(n+2))^{n+4} \delta^{-n-4} \log (4 r D) \log \log (4 r D) \\
& <2^{3(n+8)^{2}} \delta^{-n-4} \log (4 r D) \log \log (4 r D)
\end{aligned}
$$

This is our bound given for $t_{5}(n, r, D, \delta)$ in $(20.9)$.

We still have to verify that (20.11) implies (20.29). Now clearly by (20.27) and (20.23) $H(\boldsymbol{x})>n^{2 n / \delta}$ implies $Q_{1}>n^{\frac{2 n(n+2)}{\delta}}$.

As for the requirement $Q_{1}>\mathcal{H}^{1 /\left(\begin{array}{c}2 r D \\ n\end{array}\right)}$, we remark that the forms $M_{1}, \ldots, M_{2 r D}$ with which $\mathcal{H}$ is defined are just the forms $X_{1}, \ldots, X_{r}$ and $\sigma L_{i}(\sigma \in \operatorname{Gal}(\overline{\mathbb{Q}} / E), i=1, \ldots, r)$. Now $H\left(\sigma L_{i}\right)=H\left(L_{i}\right)$. On the other hand by (20.2) each of the forms defining $\mathcal{H}$ has some coefficient equal to 1.

Thus by Lemma 11.8 we get

$$
\mathcal{H}\left(M_{1}, \ldots, M_{2 r D}\right) \leq H\left(M_{1}\right) \ldots H\left(M_{2 r D}\right) .
$$

However (20.6) implies

$$
H\left(M_{i}\right) \leq H \quad \text { for } \quad i=1, \ldots, 2 r D .
$$

Therefore we may conclude that

$$
\mathcal{H} \leq H^{2 r D}
$$

On the other hand by (20.1), $r \geq n$ and therefore $\left(\begin{array}{c}2 r D \\ n\end{array}\right) \geq 2 r D$, so that

$$
\mathcal{H}^{1 /\left(\begin{array}{c}
2 r D \\
n
\end{array}\right)} \leq H^{2 r D /\left(\begin{array}{c}
2 r D \\
n
\end{array}\right)} \leq H
$$

Combining this with $(20.11)$ and $(20.23)$ we may infer that $Q>\mathcal{H}^{1 /\left(\begin{array}{c}2 r D \\ n\end{array}\right)}$. But then a fortiori $Q_{1}$ given by (20.27) satisfies the first part of (20.29). This finishes the proof of Theorem 20.1. 


\section{$21 \quad$ Proof of Theorem 3.1}

We will derive Theorem 3.1 from Theorem 20.1. Indeed we will prove that the factors in the product of the left hand side of (3.11) satisfy suitable simultaneous inequalities of the type studied in Theorem 20.1 .

So our main purpose will be to derive such simultaneous inequalities.

We will use

Lemma 21.1 Suppose $\frac{1}{2} \leq \gamma<1$ and let $q \in \mathbb{N}$. Consider the set

$$
\mathcal{J}=\left\{\left(\Gamma_{1}, \ldots, \Gamma_{q}\right) \in \mathbb{R}^{q} \mid \Gamma_{1}+\ldots+\Gamma_{q}=\gamma, \Gamma_{i} \geq 0(i=1, \ldots, q)\right\} .
$$

There exists a finite subset $\mathcal{J}^{\prime}$ of $\mathcal{J}$ of cardinality

$$
\leq\left(\frac{e}{1-\gamma}\right)^{q}
$$

with the following property:

For each point $\left(x_{1}, \ldots, x_{q}\right) \in \mathbb{R}^{q}$ having $x_{i} \geq 0(i=1, \ldots, q)$ we can find $\boldsymbol{\Gamma}=\left(\Gamma_{1}, \ldots, \Gamma_{q}\right) \in \mathcal{J}^{\prime}$ satisfying

$$
x_{i} \geq \Gamma_{i}\left(x_{1}+\ldots+x_{q}\right) \quad(i=1, \ldots, q) .
$$

A proof of Lemma 21.1 may be found e.g. in [2] (Lemma 4).

We proceed to deduce Theorem 3.1.

We partition the set of solutions $\boldsymbol{x} \in \overline{\mathbb{Q}}^{n}$ of (3.11) into two subsets $M_{1}$ and $M_{2}$. $M_{1}$ consists of those $\boldsymbol{x}$ such that

$$
\max _{\sigma \in \operatorname{Gal}(\overline{\mathbb{Q}} / E)}\left\|L_{i}^{(v)}(\sigma(\boldsymbol{x}))\right\|_{v}=0
$$

for some pair $(i, v)(v \in S, 1 \leq i \leq n)$.

$M_{2}$ consists of those solutions $\boldsymbol{x}$ which do not belong to $M_{1}$. Thus the elements $\boldsymbol{x} \in M_{2}$ will satisfy

$$
\max _{\sigma \in G a l(\overline{\mathbb{Q}} / E)}\left\|L_{i}^{(v)}(\sigma(\boldsymbol{x}))\right\|_{v} \neq 0
$$

for each pair $(i, v)(v \in S, 1 \leq i \leq n)$.

We first treat the set $M_{1}$. Given a pair $(i, v)(v \in S, 1 \leq i \leq n)$, let $T_{i v}$ be the subspace of $\overline{\mathbb{Q}}^{n}$ consisting of the vectors $\boldsymbol{y} \in \overline{\mathbb{Q}}^{n}$ with $L_{i}^{(v)}(\boldsymbol{y})=0$. In view of (3.6), $T_{i v}$ is a proper linear subspace of $\overline{\mathbb{Q}}^{n}$. Write $M_{i v}$ for the subset of points $\boldsymbol{x} \in T_{i v}$ such that we have $\sigma(\boldsymbol{x}) \in T_{i v}$ for all $\sigma \in \operatorname{Gal}(\overline{\mathbb{Q}} / E)$, and let $T_{i v}^{\prime}$ be the subspace of $\overline{\mathbb{Q}}^{n}$ generated by $T_{i v} \cap E^{n}$. Now Lemma 4.2 says that $T_{i v}^{\prime}$ is defined over $E$ and that

$$
M_{i v} \subset T_{i v}^{\prime} .
$$


Moreover by (21.4), any point $\boldsymbol{x} \in M_{1}$ satisfies for a suitable pair $(i, v)$

$$
L_{i}^{(v)}(\sigma(\boldsymbol{x}))=0 \quad \text { for all } \sigma \in \operatorname{Gal}(\overline{\mathbb{Q}} / E) .
$$

Thus $M_{1} \subset \bigcup_{v \in S} \bigcup_{i=1}^{n} T_{i v}^{\prime}$ and so $M_{1}$ may be covered by

$$
\leq n s
$$

proper linear subspaces of $\overline{\mathbb{Q}}^{n}$, all of which are defined over $E$.

We now deal with the set $M_{2}$. Write

$$
L_{i}^{(v)}(\boldsymbol{X})=\alpha_{i 1}^{(v)} X_{1}+\ldots+\alpha_{i n}^{(v)} X_{n}
$$

$(v \in S, i=1, \ldots, n)$.

Given $(i, v)(v \in S, 1 \leq i \leq n)$ pick $j=j(i, v)$ with $1 \leq j(i, v) \leq n$ such that

$$
\left\|\alpha_{i j}^{(v)}\right\|_{v}=\max \left\{\left\|\alpha_{i 1}^{(v)}\right\|_{v}, \ldots,\left\|\alpha_{i n}^{(v)}\right\|_{v}\right\} .
$$

Define the forms $L_{i}^{\prime(v)}(\boldsymbol{X})$ by

$$
L_{i}^{\prime(v)}(\boldsymbol{X})=\left(\alpha_{i j}^{(v)}\right)^{-1} L_{i}^{(v)}(\boldsymbol{X})
$$

By homogeneity we are allowed to replace the forms $L_{i}^{(v)}$ in Theorem 3.1 by the forms $L_{i}^{\prime(v)}$. Therefore we may suppose without loss of generality that for each pair $(i, v)$ $(v \in S, i=1, \ldots, n)$

$$
\text { the form } L_{i}^{(v)} \text { has } \alpha_{i, j(i, v)}^{(v)}=1
$$

and

$$
1 \leq\left\|L_{i}^{(v)}\right\|_{v} \leq n^{\frac{1}{2} s(v)}
$$

where

$$
s(v)= \begin{cases}{\left[E_{v}: \mathbb{R}\right] /[E: \mathbb{Q}]} & \text { for } v \in \mathfrak{M}_{\infty}(E) \\ 0 & \text { for } v \in \mathfrak{M}_{0}(E) .\end{cases}
$$

We now partition the set $M_{2}$ into two subsets $M_{21}$ and $M_{22}$.

$M_{21}$ consists of those $\boldsymbol{x} \in M_{2}$ for which we can find an index $i$ with $1 \leq i \leq n$ such that

$$
\prod_{v \in S} \max _{\sigma \in \operatorname{Gal}(\overline{\mathbb{Q}}, E)} \frac{\left\|L_{i}^{(v)}(\sigma(\boldsymbol{x}))\right\|_{v}}{\|\sigma(\boldsymbol{x})\|_{v}} \leq H(\boldsymbol{x})^{-n-\delta} .
$$

$M_{22}$ will be the complement of $M_{21}$ in $M_{2}$. We first treat $M_{21}$. The number of possibilities for $i$ is 
We fix $i$ and study the points $\boldsymbol{x}$ satisfying (21.13).

In view of (21.12) we have for each $v \in S$

$$
\max _{\sigma \in \operatorname{Gal}(\overline{\mathbb{Q}} / E)} \frac{\left\|L_{i}^{(v)}(\sigma(\boldsymbol{x}))\right\|_{v}}{n^{1 / 2 s(v)}\|\sigma(\boldsymbol{x})\|_{v}} \leq \max \frac{\left\|L_{i}^{(v)}\right\|\left\|_{v}\right\| \sigma(\boldsymbol{x}) \|_{v}}{n^{1 / 2} s(v)\|\sigma(\boldsymbol{x})\|_{v}} \leq 1 .
$$

Given $\boldsymbol{x}$ with $H(\boldsymbol{x})>1$ we define the tuple $\left(y_{v}\right)_{v \in S}$ by

$$
\max _{\sigma \in \operatorname{Gal}(\overline{\mathbb{Q}} / E)} \frac{\left\|L_{i}^{(v)}(\sigma(\boldsymbol{x}))\right\|_{v}}{n^{1 / 2 s(v)}\|\sigma(\boldsymbol{x})\|_{v}}=H(\boldsymbol{x})^{-y_{v}} .
$$

This is possible since $\boldsymbol{x} \notin M_{1}$. Then by (21.13) and (21.15)

$$
y_{v} \geq 0 \text { for each } v \in S, \quad \sum_{v \in S} y_{v} \geq n+\delta .
$$

We apply Lemma 21.1 with $q=s$ and with $\gamma=1-\frac{\delta}{4 n}$.

Accordingly we can cover the set of $\boldsymbol{x}$ under consideration by

$$
\left(4 e n \delta^{-1}\right)^{s}
$$

subsets with the following property:

For each subset there is a fixed tuple $\boldsymbol{\Gamma}=\left(\Gamma_{v}\right)_{v \in S}$ of nonnegative numbers with

$$
\sum_{v \in S} \Gamma_{v}=1-\frac{\delta}{4 n}
$$

such that the tuple $\left(y_{v}\right)$ in (21.16), (21.17) satisfies

$$
y_{v} \geq \Gamma_{v} \sum_{w \in S} y_{w} \geq \Gamma_{v}(n+\delta)
$$

We may conclude that for the points $\boldsymbol{x}$ in the subset corresponding to $\left(\Gamma_{v}\right)$ we have

$$
\max _{\sigma \in \operatorname{Gal}(\overline{\mathbb{Q}} / E)} \frac{\left\|L_{i}^{(v)}(\sigma(\boldsymbol{x}))\right\|_{v}}{\|\sigma(\boldsymbol{x})\|_{v}} \leq n^{1 / 2 s(v)} H(\boldsymbol{x})^{-\Gamma_{v}(n+\delta)} .
$$

Notice that (3.12) implies $n^{\frac{1}{2} s(v)}<H(\boldsymbol{x})^{\frac{\delta}{8} s(v)}$.

Combining this with (21.20) we obtain for each $v \in S$

$$
\max _{\sigma \in \operatorname{Gal}(\overline{\mathbb{Q}} / E)} \frac{\left\|L_{i}^{(v)}(\sigma(\boldsymbol{x}))\right\|_{v}}{\|\sigma(\boldsymbol{x})\|_{v}} \leq H(\boldsymbol{x})^{-\Gamma_{v}(n+\delta)+\frac{\delta}{4} s(v)} .
$$

Recall the definition of $j(i, v)$ in (21.9). For $v \in S$ we now consider the system

$$
L_{i}^{(v)}(\boldsymbol{X}), X_{k}(k=1, \ldots, n ; k \neq j(i, v)) .
$$


We denote this system by

$$
M_{1}^{(v)}(\boldsymbol{X})=L_{i}^{(v)}(\boldsymbol{X}), M_{2}^{(v)}(\boldsymbol{X}), \ldots, M_{n}^{(v)}(\boldsymbol{X}) .
$$

By (21.11) we get

$$
\left\|\operatorname{det}\left(M_{1}^{(v)}, \ldots, M_{n}^{(v)}\right)\right\|_{v}=1 .
$$

Define the tuple $\boldsymbol{e}=\left(e_{i v}\right)(v \in S, i=1, \ldots, n)$ by

$$
e_{i v}= \begin{cases}-\Gamma_{v}(n+\delta)+\frac{\delta}{8} s(v) & \text { for } i=1 \\ 0 & \text { for } i=2, \ldots, n .\end{cases}
$$

Since for $i=2, \ldots, n$ the forms $M_{i}^{(v)}(\boldsymbol{X})$ are among the coordinate forms $X_{1}, \ldots, X_{n}$ we obtain

$$
\max _{\sigma \in \operatorname{Gal}(\overline{\mathbb{Q}} / E)} \frac{\left\|M_{i}^{(v)}(\sigma(\boldsymbol{x}))\right\|_{v}}{\|\sigma(\boldsymbol{x})\|_{v}} \leq 1=H(\boldsymbol{x})^{e_{i v}}
$$

for $v \in S$ and for $i=2, \ldots, n$.

Combination of (21.21) - (21.24) yields

$$
\max _{\sigma \in \operatorname{Gal}(\overline{\mathbb{Q}} / E)} \frac{\left\|M_{i}^{(v)}(\sigma(\boldsymbol{x}))\right\|_{v}}{\left\|\operatorname{det}\left(M_{1}^{(v)}, \ldots, M_{n}^{(v)}\right)\right\|_{v}^{1 / n}\|\sigma(\boldsymbol{x})\|_{v}} \leq H(\boldsymbol{x})^{e_{i v}}
$$

for each pair $(i, v)(v \in S, i=1, \ldots, n)$.

(21.19) and (21.23) imply

$$
\begin{aligned}
\sum_{v \in S} \sum_{i=1}^{n} e_{i v} & =-(n+\delta)\left(\sum_{v \in S} \Gamma_{v}\right)+\frac{\delta}{8} \sum_{v \in S} s(v) \\
& =-(n+\delta)\left(1-\frac{\delta}{4 n}\right)+\frac{\delta}{8} \leq-n-\delta / 2
\end{aligned}
$$

and

$$
\sum_{v \in S} \max \left\{e_{1 v}, \ldots, e_{n v}\right\}=\sum_{v \in S} \frac{\delta}{8} s(v) \leq \delta / 8 .
$$

Notice that the forms $M_{i}^{(v)}$ all have a coefficient equal to 1. By (21.25) - (21.27), with $\delta$ replaced by $\delta / 2$ and with $\left\{L_{1}, \ldots, L_{r}\right\}$ replaced by $\left\{M_{i}^{(v)}: v \in S, i=1, \ldots, n\right\}$, the hypotheses of Theorem 20.1 are satisfied. Notice that $\left\{M_{i}^{(v)}: v \in S, i=1, \ldots, n\right\}$ has cardinality $\leq n s$. Therefore the set of solutions $\boldsymbol{x}$ of (21.25) with

$$
H(\boldsymbol{x})>\max \left\{H, n^{4 n / \delta}\right\}
$$

is contained in the union of not more than

$$
t_{5}=t_{5}(n, n s, D, \delta / 2) \leq 2^{3(n+8)^{2}} \delta^{-n-4} \log (4 n s D) \log \log (4 n s D)
$$


proper linear subspaces of $\overline{\mathbb{Q}}^{n}$, all of which are defined over $E$. This was for elements $\boldsymbol{x} \in M_{21}$ satisfying (21.13) with a fixed $i$ and a fixed tuple $\left(\Gamma_{v}\right)_{v \in S}$ in (21.18), (21.9). Allowing the factors from (21.14) and (21.18) for the number of possible choices of these tuples we see that

$$
n\left(4 e n \delta^{-1}\right)^{s} 2^{3(n+8)^{2}} \delta^{-n-4} \log (4 n s D) \log \log (4 n s D)
$$

subspaces will suffice to cover the set $M_{21}$.

We now deal with the set $M_{22}$.

By definition the points $\boldsymbol{x} \in M_{22}$ satisfy for each $i$

$$
\prod_{v \in S} \max _{\sigma \in \operatorname{Gal}(\overline{\mathbb{Q}} / E)} \frac{\left\|L_{i}^{(v)}(\sigma(\boldsymbol{x}))\right\|_{v}}{\|\sigma(\boldsymbol{x})\|_{v}}>H(\boldsymbol{x})^{-n-\delta} .
$$

For $v \in S$ we write

$$
\Delta_{v}=\left\|\operatorname{det}\left(L_{1}^{(v)}, \ldots, L_{n}^{(v)}\right)\right\|_{v} .
$$

Combination of (3.11) with (21.30) yields

$$
\prod_{v \in S} \Delta_{v}^{-1}<H(\boldsymbol{x})^{(n-1)(n+\delta)} .
$$

On the other hand by (21.12) we get

$$
\Delta_{v} \leq\left\|L_{1}^{(v)}\right\|_{v} \ldots\left\|L_{n}^{(v)}\right\|_{v} \leq n^{\frac{n}{2} s(v)}
$$

and therefore

$$
n^{\frac{n}{2} s(v)} \Delta_{v}^{-1} \geq 1 \quad \text { for each } v \in S .
$$

By (3.12) we have $n^{n / 2}<H(\boldsymbol{x})^{\delta / 8}$. So (21.31) implies

$$
\prod_{v \in S} n^{\frac{n}{2} s(v)} \Delta_{v}^{-1}<H(\boldsymbol{x})^{(n-1)(n+\delta)+\delta / 8} .
$$

In view of (21.32) there exists a number $A \geq 1$ such that

$$
\prod_{v \in S} n^{\frac{n}{2} s(v)} \Delta_{v}^{-1}=A^{(n-1)(n+\delta)+\delta / 8} .
$$

Moreover there exists a tuple of nonnegative numbers $\left(c_{v}\right)_{v \in S}$ with

$$
\sum_{v \in S} c_{v}=1 \quad \text { and } \quad n^{\frac{n}{2} s(v)} \Delta_{v}^{-1}=A^{c_{v}((n-1)(n+\delta)+\delta / 8)} .
$$

(21.33) implies that $A<H(\boldsymbol{x})$. Write

$$
b_{v}=\frac{1}{n} c_{v}((n-1)(n+\delta)+\delta / 8) .
$$


We then have

$$
\sum_{v \in S} \sum_{i=1}^{n} b_{v}=(n-1)(n+\delta)+\delta / 8
$$

and

$$
n^{\frac{n}{2} s(v)} \Delta_{v}^{-1 / n} H(\boldsymbol{x})^{-b_{v}} \leq 1 \quad \text { for each } v \in S .
$$

Combination of (3.11) and (21.34) yields

$$
\prod_{v \in S} \prod_{i=1}^{n} \max _{\sigma \in \operatorname{Gal}(\overline{\mathbb{Q}} / E)}\left(n^{\frac{1}{2} s(v)} \Delta_{v}^{-1 / n} H(\boldsymbol{x})^{-b_{v}} \frac{\left\|L_{i}^{(v)}(\sigma(\boldsymbol{x}))\right\|_{v}}{n^{\frac{1}{2} s(v)}\|\sigma(\boldsymbol{x})\|_{v}}\right) \leq H(\boldsymbol{x})^{-n(n+\delta)-\delta / 8} .
$$

By (21.15) and (21.35) the $n s$ factors in the double product in $(21.36)$ are all $\leq 1$. In a similar way as in the discussion of the set $M_{21}$ we may apply Lemma 21.1, now with

$$
q=n s \quad \text { and } \quad \gamma=1-\delta / 4 n^{2}
$$

consequently we obtain not more than

$$
\left(4 n^{2} e \delta^{-1}\right)^{n s}
$$

subsets of $M_{22}$, whose union equals to $M_{22}$. For each subset we have a tuple of nonnegative numbers $\boldsymbol{\Gamma}=\left(\Gamma_{i v}\right)(v \in S, i=1, \ldots, n)$ with

$$
\sum_{v \in S} \sum_{i=1}^{n} \Gamma_{i v}=\gamma=1-\delta / 4 n^{2}
$$

such that any solution $\boldsymbol{x}$ in the subset satisfies

$$
\max _{\sigma \in \operatorname{Gal}(\overline{\mathbb{Q}} / E)} H(\boldsymbol{x})^{-b_{v}} \frac{\left\|L_{i}^{(v)}(\sigma(\boldsymbol{x}))\right\|_{v}}{\Delta_{v}^{1 / n}\|\sigma(\boldsymbol{x})\|_{v}} \leq H(\boldsymbol{x})^{-\Gamma_{i v}(n(n+\delta)+\delta / 8)} \quad(v \in S, i=1, \ldots, n) .
$$

We define the tuple $\boldsymbol{e}=\left(e_{i v}\right)(v \in S, i=1, \ldots, n)$ by

$$
e_{i v}=-\Gamma_{i v}(n(n+\delta)+\delta / 8)+b_{v} .
$$

By (21.39) our solutions $\boldsymbol{x}$ under consideration satisfy

$$
\max _{\sigma \in \operatorname{Gal}(\overline{\mathbb{Q}} / E)} \frac{\left\|L_{i}^{(v)}(\sigma(\boldsymbol{x}))\right\|_{v}}{\Delta_{v}^{1 / n}\|\sigma(\boldsymbol{x})\|_{v}} \leq H^{e_{i v}} \quad(v \in S, i=1, \ldots, n) .
$$

We want to apply Theorem 20.1 with $\delta$ replaced by $\delta / 2$. So we have to check its hypotheses. By (21.34) and (21.38)

$$
\begin{aligned}
\sum_{v \in S} \sum_{i=1}^{n} e_{i v} & =-(n(n+\delta)+\delta / 2)\left(\sum_{v \in S} \sum_{i=1}^{n} \Gamma_{i v}\right)+\sum_{v \in S} \sum_{i=1}^{n} b_{v} \\
& =-\left(n^{2}+\left(n+\frac{1}{8}\right) \delta\right)\left(1-\delta / 4 n^{2}\right)+(n-1)(n+\delta)+\delta / 8 \leq-n-\delta / 2 .
\end{aligned}
$$


So the analogue of (20.7) is satisfied.

Moreover $\max _{1 \leq i \leq v} e_{i v} \leq b_{v}=\frac{1}{n} c_{v}((n-1)(n+\delta)+\delta / 8)$.

Thus

$$
\sum_{v \in S} \max _{1 \leq i \leq n} e_{i v} \leq \frac{1}{n}((n-1)(n+\delta)+\delta / 8) \sum_{v \in S} c_{v}=\frac{1}{n}((n-1)(n+\delta)+\delta / 8)<n .
$$

This means that (20.8) is satisfied as well.

For the family $\left\{L_{1}, \ldots, L_{r}\right\}$ we take $\left\{L_{i}^{(v)}: v \in S, i=1, \ldots, n\right\}$. Therefore the parameter $r$ has to be replaced by $n s$. Notice that by our normalization (21.11) also hypothesis (20.2) is satisfied. The analogue of (20.11) with $\delta$ replaced by $\delta / 2$ is

$$
H(\boldsymbol{x})>\max \left\{H, n^{4 n / \delta}\right\},
$$

but this is our assumption in (3.12). We may conclude that to cover the solutions with the fixed tuple $\left(e_{i v}\right)$ we do not need more than

$$
t_{5}(n, n s, D, \delta / 2) \leq 2^{3(n+8)^{2}} \delta^{-n-4} 2^{n+4} \log (4 n s D) \log \log (4 n s D)
$$

proper linear subspaces. Introducing the factor $\left(4 n^{2} e \delta^{-1}\right)^{n s}$ from $(21.37)$ we see that for the set $M_{22}$

$$
\left(4 n^{2} e \delta^{-1}\right)^{n s} 2^{3(n+8)^{2}+n+4} \delta^{-n-4} \log (4 n s D) \log \log (4 n s D)
$$

subspaces will suffice. By Theorem 20.1 all these subspaces may be taken such as to be defined over $E$.

Adding the bounds from (21.7), (21.29), (21.41) we may conclude that altogether we do not need more than

$$
\begin{gathered}
n s+n\left(4 e n \delta^{-1}\right)^{s} 2^{3(n+8)^{2}} \delta^{-n-4} \log (4 n s D) \log \log (4 n s D)+ \\
+\left(4 n^{2} e \delta^{-1}\right)^{n s} 2^{3(n+8)^{2}+n+4} \delta^{-n-4} \log (4 n s D) \log \log (4 n s D) \\
<2 \cdot\left(4 n^{2} e \delta^{-1}\right)^{n s} 2^{3(n+8)^{2}+n+4} \delta^{-n-4} \log (4 n s D) \log \log (4 n s D) \\
<(3 n)^{2 n s} 2^{3(n+9)^{2}} \delta^{-n s-n-4} \log (4 D) \log \log (4 D)
\end{gathered}
$$

subspaces, and this is the bound given for $t_{2}(n, s, D, \delta)$ in $(3.10)$.

\section{References}

[1] E. Bombieri and J. Vaaler. On Siegel's lemma. Invent. Math. 73 (1983), 11-32.

[2] J.-H. Evertse. On equations in S-units and the Thue-Mahler equation. Invent. Math. 75 (1984), 561-584. 
[3] J.-H. Evertse. An explicit version of Faltings' Product theorem and an improvement of Roth's lemma. Acta Arith. 73 (1995), 215-248.

[4] J.-H. Evertse. An improvement of the quantitative Subspace theorem. Compositio Math. 101 (1996), 225-311.

[5] J.-H. Evertse, H. P. Schlickewei and W. M. Schmidt. Linear equations in variables which lie in a multiplicative group. To appear.

[6] G. Faltings. Diophantine approximation on abelian varieties. Annals of Math. 133 (1991), 549-576.

[7] R. B. McFeat. Geometry of numbers in adèle spaces. Dissertationes Mathematicae 88, PWN Polish Scient. Publ., Warsaw (1971).

[8] D. Roy and J. L. Thunder. An absolute Siegel's Lemma. J. reine angew. Math. 476 (1996), 1-26.

[9] H. P. Schlickewei. The quantitative Subspace Theorem for number fields. Compositio Math. 82 (1992), 245-273.

[10] H. P. Schlickewei. Multiplicities of recurrence sequences. Acta Math. 176 (1996), $171-243$.

[11] H. P. Schlickewei. Linear equations over finitely generated groups. Preprint (1994).

[12] W. M. Schmidt. Norm form equations. Annals of Math. 96 (1972), 526-551.

[13] W. M. Schmidt. Diophantine approximation. Springer Lecture Notes in Math. 785, Berlin, Heidelberg, New-York (1980).

[14] W. M. Schmidt. The subspace theorem in diophantine approximation. Compositio Math. 96 (1989), 121-173.

[15] S. Zhang. Positive line bundles on arithmetic varieties. J. Amer. Math. Soc. 8 (1995), 187-221.

J.-H. Evertse

Universiteit Leiden, Mathematisch Instituut

Postbus 9512, 2300 RA Leiden, The Netherlands

e-mail: evertse@math.leidenuniv.nl

H. P. Schlickewei

Fachbereich Mathematik und Informatik

Philipps-Universität Marburg

Lahnberge, Hans-Meerwein-Straße 
35032 Marburg

Germany

e-mail: hps@mathematik.uni-marburg.de 